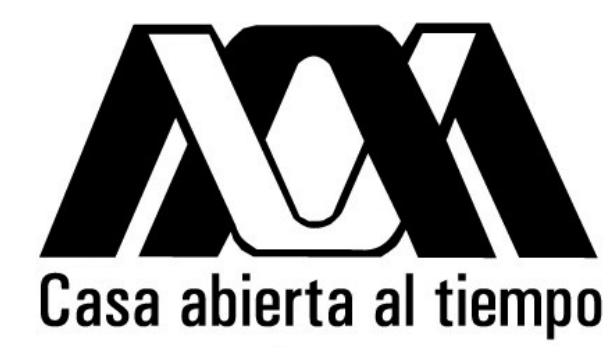

UNIVERSIDAD AUTÓNOMA METROPOLITANA

Universidad Autónoma Metropolitana-Iztapalapa

División de Ciencias Básicas e Ingeniería

\title{
La Estadística en el Mantenimiento y Reemplazo Óptimo en el Control de Calidad
}

\author{
Tesis \\ Que para obtener el grado de: \\ Maestro en Ciencias (Matemáticas Aplicadas e Industriales) \\ Presenta \\ Raquel Vergara Lazcano
}

Asesor

Dr. Alberto Castillo Morales

México D.F., Febrero 2014 


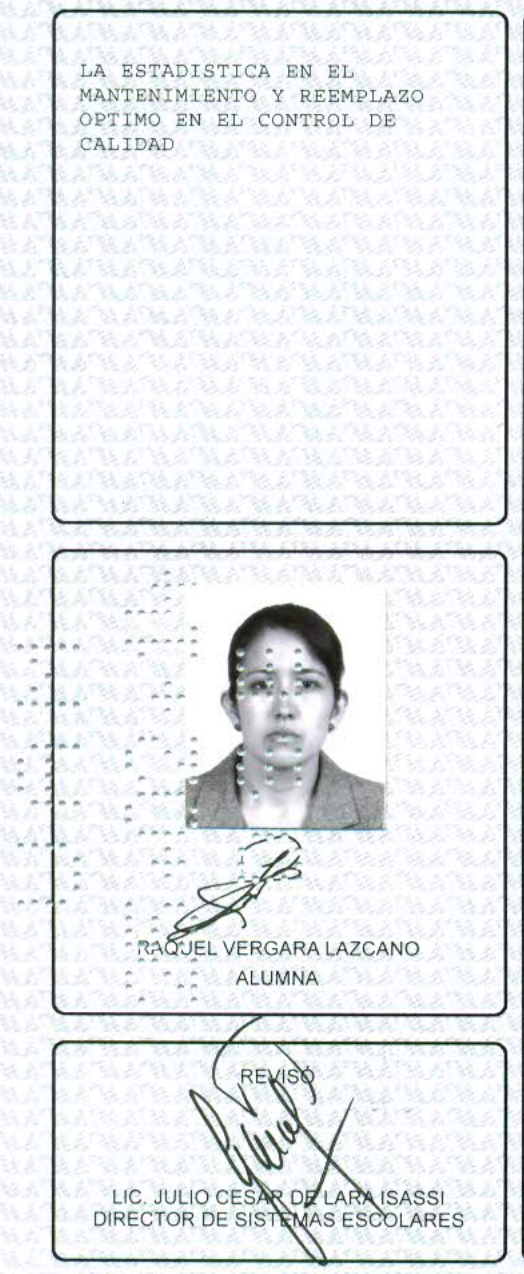

En México, D.F., se presentaron a las 12:15 horas del día 20 del mes de febrero del año 2014 en la Unidad Iztapalapa de la Universidad Autónoma Metropolitana, los suscritos miembros del jurado:

DR. GABRIEL ESCARELA PEREZ

MTRA, ELVIRA BEATRIZ CLAVEL DIAZ

DR. ALBERTO CASTILLO MORALES

Bajo la presidencia del primero y con carácter de Secretario el ultimo, se reunieron para proceder al Examen de Grado cuya denominación aparece al margen, para la obtención del grado de:

MAESTRA EN CIENCIAS (MATEMÁTICAS APLICADAS E INDUSTRIALES) DE: RAQUEL VERGARA LAZCANO

y de acuerdo con el artículo 78 fracción III del Reglamento de Estudios Superiores de la Universidad Autónoma Metropolitana, resolvieron:

\section{Aprobar}

Acto continuo, el presidente del jurado comunicó a la interesada el resultado de la evaluación $y$, en caso aprobatorio, le fue tomada la protesta.

DIRECTOR DE LA DIVISIÓN DE CBI

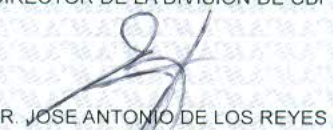

HEREDIA

VOCAL

Buatri \&. ¿Goul \&. MTRA. ELVIRA BEATRIZ CLAVEL DIAZ
PRESIDENTE

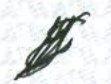

DR. GABRIEL ESCARELA PEREZ

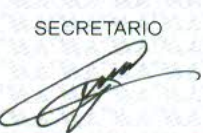

DR. ALBERTO CASTILLO MORALES 


\section{Dedicatoria}

Con todo cariño, a mi familia. 



\section{Agradecimientos}

En primer lugar quiero agradecer a mi asesor, el Dr. Alberto Castillo Morales por haberme brindado la oportunidad de realizar esta tesis. Agradezco su continuo apoyo y el esfuerzo que ha realizado para que este trabajo se pudiera concluir.

También agradezco a mis sinodales, por sus aportaciones realizadas para mejorar y complementar este trabajo. 



\section{Índice general}

Dedicatoria

Agradecimientos $\quad$ V

$\begin{array}{ll}\text { Objetivos } & 1\end{array}$

\begin{tabular}{ll}
\hline Introducción & 3
\end{tabular}

1. Conceptos básicos de confiabilidad 5

1.1. Definiciones preliminares . . . . . . . . . . . . . . . 5

1.1.1. Función de densidad de probabilidad . . . . . . . . . . . 5

1.1.2. Función de distribución acumulada . . . . . . . . . . . . . 6

1.1.3. Función de confiabilidad . . . . . . . . . . . . . . . . 6

1.1.4. La tasa de falla . . . . . . . . . . . . . . . . 7

1.1.5. Tasa de falla acumulada . . . . . . . . . . . . . . . 9 9

1.1.6. El valor medio de los tiempos de falla . . . . . . . . . . . . . . 9

1.1.7. Tasa de fallo promedio . . . . . . . . . . . . . . . . . 10

1.1.8. El p-cuantil . . . . . . . . . . . . . . . . . . . . 11

1.1.9. Estadísticos de orden . . . . . . . . . . . . . . . . . . . . 12

1.2. Relaciones y equivalencias . . . . . . . . . . . . . . . . . . . 12

1.3. Modelos de distribución en Confiabilidad . . . . . . . . . . . . . . . . 13

1.3.1. Distribución Exponencial . . . . . . . . . . . . . . . . . . 13

1.3.2. Distribución Weibull . . . . . . . . . . . . . . . . 15

1.3.3. Distribución Normal . . . . . . . . . . . . . . . . . . . 16

1.3.4. Distribución Lognormal . . . . . . . . . . . . . . . . . 20

1.4. Censura de datos . . . . . . . . . . . . . . . . . . . . . . . 22

1.5. Estimación de parámetros por método de Máxima Verosimilitud (MV) 23

1.5.1. Estimación del parámetro de la distribución Exponencial . . . 24

1.6. Estimación no paramétrica . . . . . . . . . . . . . . . . . 26 
1.6.1. Estimación de la función de distribución en caso de observaciones completas . . . . . . . . . . . . . 26

1.6.2. Estimación de la función de confiabilidad en caso de observaciones censuradas. . . . . . . . . . . . . . . . . . 27

1.7. Estimación de parámetros mediante

linealización de la función de distribución. . . . . . . . . . . . . . . . . . 29

1.8. Modelos de vida acelerada . . . . . . . . . . . . . . . . . . . 34

1.8.1. Distribución del tiempo de falla, bajo vida acelerada . . . . . . . . . . . . . . . . . . . . . 34

1.8.2. Modelo de Arrhenius . . . . . . . . . . . . . . . . . . . 37

1.8.3. Modelo de Eyring . . . . . . . . . . . . . . . . . . . . . . . . . . . . . . . . 39

1.8.4. Ley de la Potencia Inversa . . . . . . . . . . . . . . . . . . . . 40

2. Mantenimiento 43

2.1. Tipos de Mantenimiento . . . . . . . . . . . . . . . . . . . . . . . . . . . . . . . . 44

2.2. Mantenimiento Correctivo . . . . . . . . . . . . . . . . . . . . . . . . . . . . . 44

2.3. Mantenimiento Preventivo . . . . . . . . . . . . . . . . . . . . . . 45

2.4. Función de densidad de fallo que resulta cuando se practican acciones de mantenimiento preventivo . . . . . . . . . . . . . . . . . 46

2.5. Función de Mantenibilidad . . . . . . . . . . . . . . . . . . . . . . . 51

3. Modelos Matemáticos de Políticas Óptimas de Mantenimiento con tiempos de reparación instantáneos $\quad 55$

3.1. Política I. Mantenimiento Basado en la Edad . . . . . . . . . . . . . . 56

3.2. Política II. Mantenimiento preventivo a intervalos constantes . . . . . 61

3.3. Aplicación a un caso real . . . . . . . . . . . . . . . . . . . 66

4. Políticas óptimas de mantenimiento preventivo para sistemas con $\begin{array}{ll}\text { varios componentes. } & 73\end{array}$

4.1. Confiabilidad de sistemas en serie . . . . . . . . . . . . . . . . . . . . 74

4.1.1. Tasa de falla del sistema . . . . . . . . . . . . . . 75

4.1.2. Sistema en serie con componentes de distribución exponencial 76

4.2. Política de reemplazo por edad para un sistema en serie . . . . . . . . 77

4.3. Política de reemplazo a intervalos constantes para un sistema en serie 80

4.4. Confiabilidad de sistemas en paralelo . . . . . . . . . . . . . . 81

4.4.1. Sistema en paralelo con componentes de distribución exponencial 83

4.5. Política de reemplazo por edad para un sistema en paralelo . . . . . . 85

4.6. Política de reemplazo a intervalos constantes para un sistema en paralelo 87

4.7. Sistemas Mixtos . . . . . . . . . . . . . . . . . . . . 88

4.8. Mantenimiento Oportunista para Sistemas en Serie . . . . . . . . . . 92

4.8.1. Descripción del Sistema . . . . . . . . . . . . . . . . . . . . 93

4.8.2. Estructura de costos . . . . . . . . . . . . . . . . . . 93 
4.8.3. Descripción del modelo . . . . . . . . . . . . . . . . . . . . 95

4.9. Conclusiones . . . . . . . . . . . . . . . . . . . 100 
XII 


\section{Objetivos}

\section{Objetivos generales}

El objetivo general de esta tesis es presentar y realizar los análisis estadísticos correspondientes a los tiempos falla de un sistema con uno o más componentes, para poder realizar una adecuada planeación de los tiempos en los cuales se debe efectuar el mantenimiento preventivo de los componentes del sistema. Para lograr esto último, se van a aplicar distintas políticas de mantenimiento preventivo: Mantenimiento Basado en la Edad, Mantenimiento a Intervalos Constantes y Mantenimiento Oportunista para sistemas en serie. Estas tres políticas tienen por objetivo determinar el intervalo de reemplazo óptimo de los componentes de un sistema a fin de minimizar los costos totales asociados al mantenimiento.

\section{Objetivos particulares}

Los objetivos particulares de la tesis son los siguientes:

- Realizar un estudio de los modelos más usados que se ajustan a la distribución de los tiempos de falla, y a partir de cada uno éstos medir algunas de las funciones básicas del área de Confiabilidad como son: función de confiabilidad, tasa de falla y tiempo medio entre fallas.

- Estudiar los procedimientos que permitan realizar una estimación paramétrica de la función de confiabilidad a partir de una muestra dada, independientemente de si los datos de la muestra estén completos o presenten algún tipo de censura.

- Estudiar los procedimientos a partir de los cuales se puede realizar una estimación no paramétrica de la función de confiabilidad tanto para observaciones completas como para observaciones censuradas por la derecha.

- Estudiar la función de densidad de falla que resulta cuando se efectúan actividades de mantenimiento preventivo en intervalos periódicos. 
- Estudiar las funciones básicas del área de confiabilidad para sistemas con más de dos componentes, ya sea que se trate de una configuración en serie, paralela o mixta.

- Estudiar las políticas de mantenimiento preventivo de los elementos de un sistema: Mantenimiento Basado en la Edad, Mantenimiento a Intervalos Constantes y Mantenimiento Oportunista para Sistemas en Serie. 


\section{Introducción}

Hoy en día, el rápido avance tecnológico, el desarrollo de productos altamente sofisticados, el incremento de las perspectivas del consumidor y la intensa competencia global, han obligado a las empresas a mejorar la calidad de sus productos o servicios. Pero, ¿qué significa calidad? Calidad significa cumplir con los requerimientos especificados en un producto o servicio, para satisfacer las necesidades del cliente. También se puede entender como las propiedades de una cosa que permiten apreciarla como igual, mejor o peor que cualquier otra de su misma especie. Sin embargo, cada vez es más importante la extensión de la calidad en el tiempo de uso del producto o servicio, y es aquí donde aparece el concepto de confiabilidad. La confiabilidad intenta garantizar que el producto realizará adecuadamente su función durante un periodo razonable de tiempo.

Todo usuario sabe que independientemente de la perfección del diseño de un sistema, de la tecnología de su producción o de los materiales usados en su fabricación, a lo largo de su operación se producirán cambios irreversibles, producto del desgaste u obsolescencia de los materiales. La desviación de esas características respecto a los valores especificados es lo que se considera como falla del sistema.

Por consiguiente, la falla del sistema se puede definir como un suceso cuya realización provoca, ya sea la pérdida de capacidad para realizar las funciones requeridas, o bien la pérdida de capacidad para satisfacer los requisitos especificados.

Hay muchos sistemas para los cuales se puede recuperar capacidad de funcionamiento y se les denomina sistemas recuperables. Para que un sistema recupere la capacidad de realizar una función es necesario realizar ciertas actividades, conocidas como actividades de mantenimiento.

Existen distintos tipos de mantenimiento: correctivo, preventivo, proactivo y mantenimiento basado en la condición; éste último ayuda a predecir la falla de un sistema a través del monitoreo de ciertas características del sistema. 
En esta tesis sólo se estudiarán el mantenimiento correctivo y preventivo, los cuales en conjunto ayudarán a predecir un intervalo de mantenimiento preventivo óptimo para una unidad o sistema.

En el Capítulo 1, se inicia con un estudio de los conceptos básicos del área de Confiabilidad Estadística como son: función de confiabilidad, tasa de falla y tiempo medio para la falla; se definen cada uno de éstos para algunos de los modelos de distribución de tiempos a la falla: distribución Exponencial, distribución Weibull, distribución Normal y distribución Lognormal. Se realiza un estudio de la estimación de parámetros por Método de Máxima Verosimilitud, así como de la estimación no paramétrica, tanto para observaciones completas, como para observaciones censuradas. También se presentan algunos de los modelos de vida acelerada como son: Modelo de Arrhenius, Modelo de Eyring y Modelo de la Potencia Inversa.

El Capítulo 2 trata cuestiones relacionadas con el mantenimiento de un sistema. Se presentan las definiciones de los conceptos de mantenimiento correctivo y preventivo y se expone la función de densidad que resulta cuando se realizan actividades de mantenimiento preventivo.

El Capítulo 3 se dedica al estudio de las políticas de mantenimiento preventivo para un solo componente: reemplazo por edad y reemplazo a intervalo constantes. Estas políticas indican el intervalo de tiempo óptimo para llevar a cabo el reemplazo o mantenimiento periódico de un cierto componente al menor costo. Se presentan algunos ejemplos que ayudarán a comprender cada una de las políticas.

En lo que se refiere al Capítulo 4, se presentan los conceptos de confiabilidad para las distintas configuraciones de un sistema: sistema en serie, sistema en paralelo y sistema mixto. Nuevamente se exponen las políticas de mantenimiento preventivo que se estudiaron en el caso de un solo componente, pero ahora en el caso de un sistema con múltiples componentes, considerando que éstos forman parte de alguna de las configuraciones que se mencionaron anteriormente. En esta parte, las políticas de mantenimiento indicarán el intervalo de tiempo para reemplazar todos los componentes del sistema. Este último enfoque presenta desventajas económicas, sobre todo cuando el sistema está formado por componentes con distintas tasas de falla, pues se realizará el reemplazo de componentes que aún continúan funcionando. Una alternativa a este método es la política de mantenimiento oportunista, la cual considera la falla de un sistema como una oportunidad para realizar el reemplazo preventivo de los componentes que no fallaron en ese momento. El objetivo de ésta es indicar cuándo y qué componentes de un sistema reemplazar a fin de minimizar los costos totales de mantenimiento. Al final del Capítulo 4 se presenta la política de mantenimiento oportunista para un sistema en serie con dos componentes. 


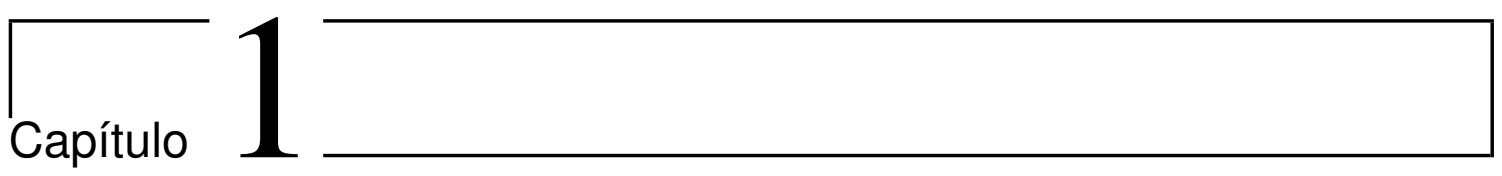

\section{Conceptos básicos de confiabilidad}

En este primer capítulo se presentan los conceptos básicos utilizados en el análisis de confiabilidad, así como algunos de los modelos de distribución utilizados para modelar tiempos a la falla.

\subsection{Definiciones preliminares}

Se dice que una falla ocurre cuando un componente o unidad deja de funcionar como es requerido. Considérese a $T$ como una variable aleatoria que representa el tiempo a la falla de un componente o sistema, la cual toma valores en el intervalo $[0,+\infty)$; ésta puede ser tiempo real, número de horas de vuelo, número de ciclos de operación, etc.

\subsubsection{Función de densidad de probabilidad}

La función de densidad $f$ de la variable aleatoria $T$ se define como la probabilidad que tiene un componente de fallar en un instante $t$. Para esta función continua se cumple que:

- $f(t) \geq 0, \quad \forall t \in[0,+\infty)$

- $\int_{0}^{\infty} f(t) d t=1$ 


\subsubsection{Función de distribución acumulada}

La función de distribución acumulada $F$ de la variable aleatoria $T$, representa la probabilidad acumulada de fallo hasta el tiempo $t$, es decir

$$
F(t)=P(T \leq t), \quad \forall t \in[0,+\infty)
$$

La función $F(\cdot)$ es una función continua y monótona no decreciente, para la cual se verifica que

- $\lim _{t \rightarrow-\infty} F(t)=0$

- $\lim _{t \rightarrow \infty} F(t)=1$

A partir de la función de distribución $F(t)$, se puede definir la función de densidad $f(t)$ como

$$
f(t)=F^{\prime}(t)=\frac{d}{d t} F(t)
$$

\subsubsection{Función de confiabilidad}

La confiabilidad se define como la probabilidad de que una unidad o componente realice la función para la cual fue diseñado, bajo ciertas condiciones de uso especificadas, por un periodo de tiempo determinado. Las condiciones de uso son importantes para definir la confiabilidad, por ejemplo: no duran los mismo unos neumáticos si se usan en autopista, que si se usan en un camino rural.

La función de confiabilidad $R(t)$, es la probabilidad de que la variable aleatoria $T$ sea mayor a $t$ :

$$
R(t)=P(T>t)=\int_{t}^{\infty} f(u) d u=1-F(t), \quad \forall t \in[0,+\infty)
$$

Esta función es continua y monótona decreciente. Para ésta se cumple que:

- $R(0)=1$

- $R(\infty)=\lim _{t \rightarrow \infty}(1-F(t))=0$ 
Por su definición, la función de confiabilidad se utiliza en otras disciplinas tales como: Medicina, Actuaría o Finanzas en las que se realizan análisis de supervivencia. En estas disciplinas, la función de confiabilidad se conoce como función de supervivencia.

Dentro de las finanzas, el análisis de supervivencia tiene aplicaciones en diversas áreas, en particular en la de seguros, ya sean de vida, de autos, de casas o de desempleo. Por ejemplo, para calcular el monto las primas de seguros de vida es importante realizar un estudio de las tasas de mortalidad de los asegurados. Entre otros estudios, se analiza la probabilidad de muerte en las diferentes edades dadas ciertas condiciones de salud; a partir de este análisis, es fácil calcular la probabilidad de que el asegurado fallezca durante la vigencia de la póliza del seguro de vida.

\subsubsection{La tasa de falla}

Otra de las funciones importantes para modelar tiempos a la falla es la función de riesgo o tasa de falla $h(t)$. Esta función representa la probabilidad instantánea, por unidad de tiempo, que tiene un componente de fallar en un instante $t$, dado que había funcionado hasta el instante anterior. Analíticamente se expresa por la razón:

$$
h(t)=\frac{f(t)}{R(t)}
$$

siempre que $R(t) \neq 0$.

Esta razón se puede interpretar como la proporción de fallas en el instante $t$ relativo sólo a los componentes que no han fallado hasta ese instante. A diferencia de esta función, la función de densidad $f(t)$ representa la proporción de fallas en el instante $t$, pero respecto al total de la muestra.

La tasa de falla se obtiene a partir del límite, cuando $\Delta t \rightarrow 0$, de la probabilidad condicionada de que el componente falle antes del tiempo $t+\Delta t$, dado que había 
funcionado hasta el instante $t$. De esta forma

$$
\begin{aligned}
h(t) & =\lim _{\Delta t \rightarrow 0} \frac{P(t<T \leq t+\Delta t \mid T>t)}{\Delta t} \\
& =\lim _{\Delta t \rightarrow 0} \frac{P(t<T \leq t+\Delta t, T>t) / P(T>t)}{\Delta t} \\
& =\lim _{\Delta t \rightarrow 0} \frac{P(t<T \leq t+\Delta t)}{\Delta t P(T>t)} \\
& =\frac{1}{R(t)} \lim _{\Delta t \rightarrow 0} \frac{F(t+\Delta t)-F(t)}{\Delta t} \\
& =\frac{f(t)}{R(t)}
\end{aligned}
$$

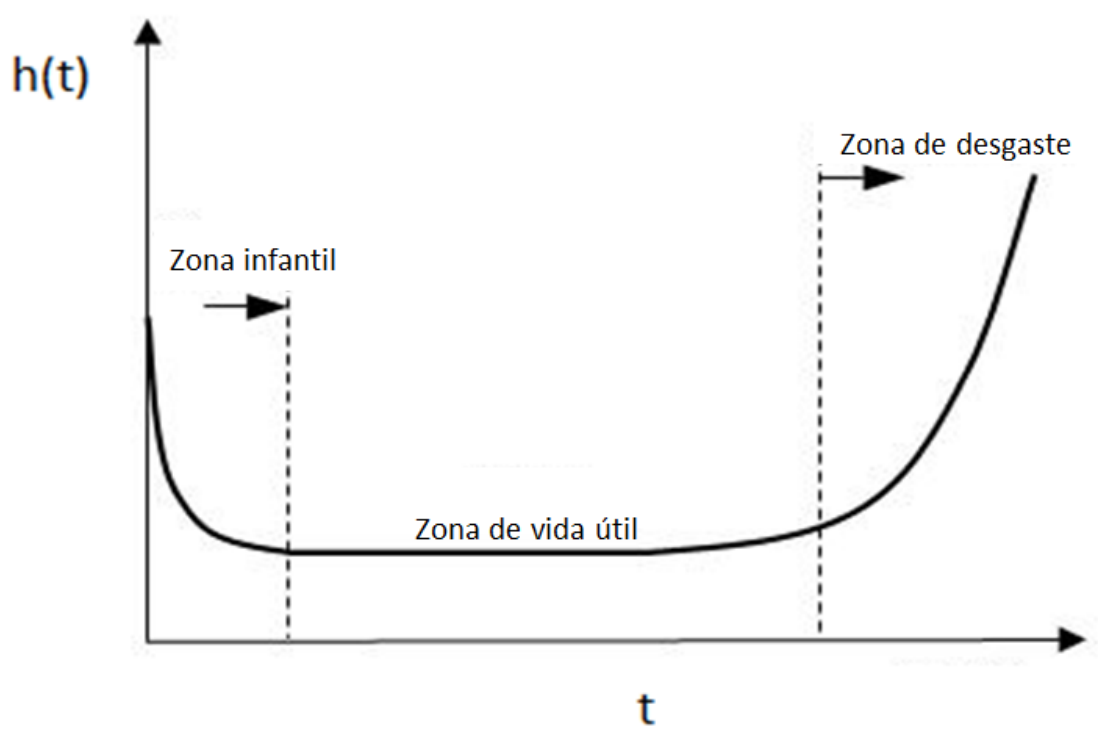

Figura 1.1: Curva de la Bañera

Los expertos de confiabilidad, en algunos casos, utilizan la curva de la bañera para describir el comportamiento de la tasa de falla en los componentes o sistemas [?]. En la Figura 1.1 se muestra la gráfica de la función que caracteriza la tasa de falla a través del tiempo. Se muestran tres zonas: zona infantil, zona de vida útil y zona de desgaste. En la primer zona, de corta duración, se puede apreciar una tasa de falla alta pero decreciente, se observa en componentes cuya probabilidad de fallo es menor a medida que aumenta el tiempo. Aquí las fallas aparecen inmediatamente 
y al cabo de poco tiempo de poner en funcionamiento el sistema; la mayoría de las veces, como consecuencia de defectos de fabricación. En la segunda zona se observa una tasa de falla constante; esta indica que la probabilidad de falla instantánea es la misma en cualquier momento, es decir, el proceso no tiene memoria, ya que la posibilidad de falla estando funcionando es idéntica en cualquier momento de la vida del sistema. Las fallas en esta zona son originadas por causas inexplicables y fenómenos naturales imprevistos. En la zona de desgaste se observa una tasa de falla

creciente. Ésta se presenta en la mayoría de los casos por desgastes u obsolescencia del ítem, es decir, por un proceso de envejecimiento. La tasa de falla creciente en la zona de desgaste indica que la probabilidad de falla instantánea, teniendo en cuenta que el componente está funcionando, se incrementa a medida que aumenta el tiempo.

\subsubsection{Tasa de falla acumulada}

Una vez definida la tasa de falla, se define la tasa de falla acumulada $H(t)$, que como su nombre lo indica, acumula la tasa de falla a lo largo del tiempo.

$$
H(t)=\int_{0}^{t} h(u) d u .
$$

\subsubsection{El valor medio de los tiempos de falla}

Se pueden considerar dos situciones, dependiendo de la unidad o dispositivo que se trate.

Si se considera un dispositivo o sistema el cual una vez que falla es restaurado a su estado de funcionamiento, se define el tiempo medio entre falla, en inglés $M T B F$ (Medium Time Between Failure). Matemáticamente se tiene

$$
M T B F=\int_{0}^{\infty} t f(t) d t
$$

El MTBF también se puede expresar en términos de la función de confiabilidad,

$$
M T B F=\int_{0}^{\infty} R(t) d t
$$




\section{Demostración}

Realizamos integración por partes en $\int_{0}^{\infty} t f(t) d t$ Hacemos

$$
\begin{array}{rlrl}
u & =t & d v & =f(t) d t \\
d u & =d t & v & =-R(t)
\end{array}
$$

Entonces queda que

$$
\begin{aligned}
M T B F & =\int_{0}^{\infty} t f(t) d t \\
& =-\left.t R(t)\right|_{0} ^{\infty}+\int_{0}^{\infty} R(t) d t
\end{aligned}
$$

Para el primer término de la última igualdad, se tiene que $t R(t)$ es cero cuando $t=0$, de ahí que sólo nos queda por evaluar $t R(t)$ cuando $t=\infty$, de lo cual resulta el siguiente límite

$$
\lim _{t \rightarrow \infty} t R(t)
$$

Como el anterior es un límite indeterminado, usamos la Ley de L'Hopital, para tener

$$
\begin{aligned}
\lim _{t \rightarrow \infty} \frac{t}{R(t)^{-1}} & =\lim _{t \rightarrow \infty} \frac{1}{R(t)^{-2} f(t)} \\
& =\lim _{t \rightarrow \infty} \frac{R(t)}{h(t)}
\end{aligned}
$$

Siendo $h(t)=\frac{f(t)}{R(t)}$

El valor del último límite es nulo siempre que $h(t)$ no sea cero. El hecho de que se tenga que para un componente o sistema que está funcionando el $\lim _{t \rightarrow \infty} h(t)=0$, nos conduce a pensar que el valor de la tasa de falla tiende cada vez más a cero, lo cual significaría que el componente o sistema se perfecciona a medida que pasa el tiempo, cosa que no sucede. En otras palabras, el sistema debe fallar después de una cantidad finita de tiempo funcionando. Por este motivo se deduce que $\lim _{t \rightarrow \infty} h(t) \neq 0$.

Finalmente

$$
\int_{0}^{\infty} t f(t) d t=\int_{0}^{\infty} R(t) d t
$$

En el otro caso, si el dispositivo no es reparado una vez que falla, como es el caso de un foco, es usual definir el tiempo medio para la falla, en inglés MTTF (Medium 
Time To Failure). Al igual que el $M T B F$, si $R_{s r}(t)$ indica la confiabilidad del sistema que es no restaurado una vez que falla, se puede escribir

$$
M T T F=\int_{0}^{\infty} R_{s r}(t) d t
$$

\subsubsection{Tasa de fallo promedio}

Otra definición de gran interés es la tasa de fallo promedio entre los instantes $t_{1}$ y $t_{2}$, que denotaremos por $A F R\left(t_{1}, t_{2}\right)$,

$$
\operatorname{AFR}\left(t_{1}, t_{2}\right)=\frac{\int_{t_{1}}^{t_{2}} h(u) d u}{t_{2}-t_{1}}
$$

\subsubsection{El p-cuantil}

El $p$-cuantil de la distribución de la variable aleatoria $T$ es el valor $t_{p}$ de la variable que separa una probabilidad acumulada $p$ a la izquierda. De este modo, el $p$-cuantil $t_{p}$ verifica que

$$
P\left(T \leq t_{p}\right)=p
$$

Luego, si $F$ es la función de distribución de la variable aleatoria, el $p$-cuantil se obtiene como solución de la ecuación

$$
F\left(t_{p}\right)=p
$$

Es decir, $t_{p}=F^{-1}(p)$, donde $F^{-1}$ es la función inversa de $F$.

En la práctica es de gran interés el tiempo de falla mediano $t_{0.5}$, que es el menor punto donde la función de distribución acumulada alcanza el valor de 0.5.

Ejemplo 1.1.1. Calcular el tiempo de falla mediano, suponiendo que el tiempo de falla es modelado por la distribución exponencial, cuya función de distribución es $F(t)=1-\exp (-\lambda t), \quad t \geq 0, \quad \lambda>0$.

Queremos encontrar el valor de $t_{0.5}$ para el cual se verifica que 


$$
\begin{aligned}
1-\exp \left(-\lambda t_{0.5}\right) & =0.5 \\
\exp \left(-\lambda t_{0.5}\right) & =0.5 \\
-\lambda t_{0.5} & =\ln (0.5) \\
t_{0.5} & =\frac{\ln 2}{\lambda}
\end{aligned}
$$

Observe que el tiempo de falla mediano, $t_{0.5}=\frac{\ln 2}{\lambda}$, que indica que el $50 \%$ de los componentes habrán fallado cuando se alcanza este valor, es menor que el correspondiente $M T T F$, que es $\frac{1}{\lambda}$. Para este último valor se tiene que la distribución acumulada, es decir, $F(M T T F)=1-e^{-1} \approx 0.632$, lo cual significa que aproximadamente el $63.2 \%$ de los componentes habrán fallado cuando se alcanza el MTTF.

\subsubsection{Estadísticos de orden}

En algunos estudios, como los ensayos de vida, en los que se analiza el tiempo de falla o duración de ciertos componentes, los datos correspondientes a los tiempos de falla de un conjunto de componentes son más relevantes si se presentan en un orden no decreciente.

De esta forma, si $t_{(1)} \leq t_{(2)} \leq t_{(n)}$ representa la muestra $t_{1}, t_{2}, \ldots, t_{n}$ ordenada de menor a mayor, entonces $t_{(k)}$ es el estadístico de orden $k$-ésimo. A los estadísticos $t_{(1)}$ y $t_{(n)}$ se les llama mínimo y máximo respectivamente.

\subsection{Relaciones y equivalencias}

De las funciones que se definieron anteriormente, se pueden establecer las siguientes relaciones.

1. Relación entre la función de densidad, función de distribución y función de 
confiabilidad.

$$
\begin{aligned}
f(t) & =\frac{d}{d t} F(t) \\
f(t) & =\frac{d}{d t}(1-R(t)) \\
f(t) & =-R^{\prime}(t)
\end{aligned}
$$

2. Relación entre tasa de falla y función de confiabilidad

$$
\begin{aligned}
h(t) & =-\frac{R^{\prime}(t)}{R(t)} \\
& =-\frac{d}{d t} \ln R(t)
\end{aligned}
$$

3. Relación entre tasa de falla acumulada y función de confiabilidad.

$$
\begin{aligned}
H(t) & =\int_{0}^{t} h(u) d u \\
& =\int_{0}^{t} \frac{d}{d u}-\ln R(u) d u \\
& =-\ln R(t)
\end{aligned}
$$

Por tanto

$$
\begin{aligned}
R(t) & =e^{-\int_{0}^{t} h(u) d u} \\
& =e^{-H(t)}
\end{aligned}
$$

4. Relación entre función de densidad y tasa de falla

$$
\begin{aligned}
f(t) & =h(t) e^{-\int_{0}^{t} h(u) d u} \\
& =h(t) e^{-H(t)} \\
& =h(t) R(t)
\end{aligned}
$$

La importancia de conocer las relaciones y equivalencias entre las funciones $f$, $h, F$ y $R$, se deriva del hecho de que si sólo se conoce una de estas funciones, se pueden deducir cualesquiera de las otras. 


\subsection{Modelos de distribución en Confiabilidad}

En la literatura se consideran los modelos que mejor han ajustado en los pro-

blemas de confiabilidad. Éstos coinciden con los modelos de tiempo de vida en el Análisis de Supervivencia.

\subsubsection{Distribución Exponencial}

Esta distribución es utilizada para modelar tiempos de falla que presentan una tasa de falla constante, es decir, que la probabilidad de fallo, condicionada a que el elemento o componente esté en uso no varía con el tiempo. Los sistemas electrónicos como por ejemplo, sistemas de generación de estados de cuenta bancarios y cajeros automáticos son sistemas cuyos tiempos de falla se modelan con esta distribución.

Función de densidad

$$
f(t)=\lambda \exp (-\lambda t), \quad t \geq 0, \quad \lambda>0
$$

Función de distribución

$$
F(t)=1-\exp (-\lambda t), \quad t \geq 0, \quad \lambda>0
$$

Función de confiabilidad

$$
R(t)=\exp (-\lambda t), \quad t \geq 0, \quad \lambda>0
$$

Tasa de falla

$$
h(t)=\lambda, \quad t>0
$$

Tiempo medio entre falla(MTBF)

$$
M T B F=\frac{1}{\lambda}
$$

Función p-cuantil

$$
t_{p}=\frac{-\ln (1-p)}{\lambda}
$$




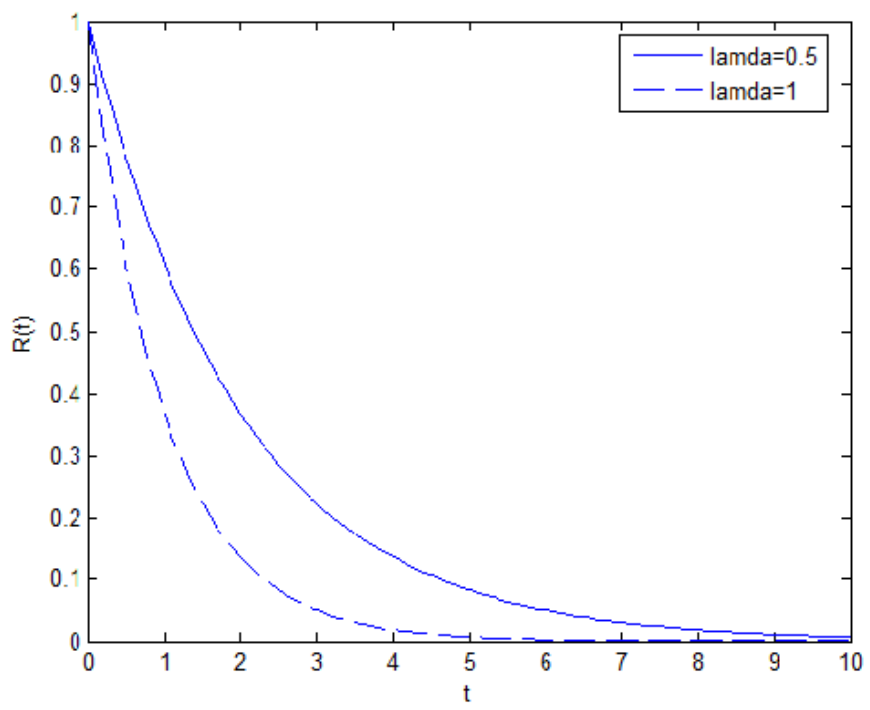

Figura 1.2: Función de confiabilidad de la distribución Exponencial con $\lambda=0.5,1$

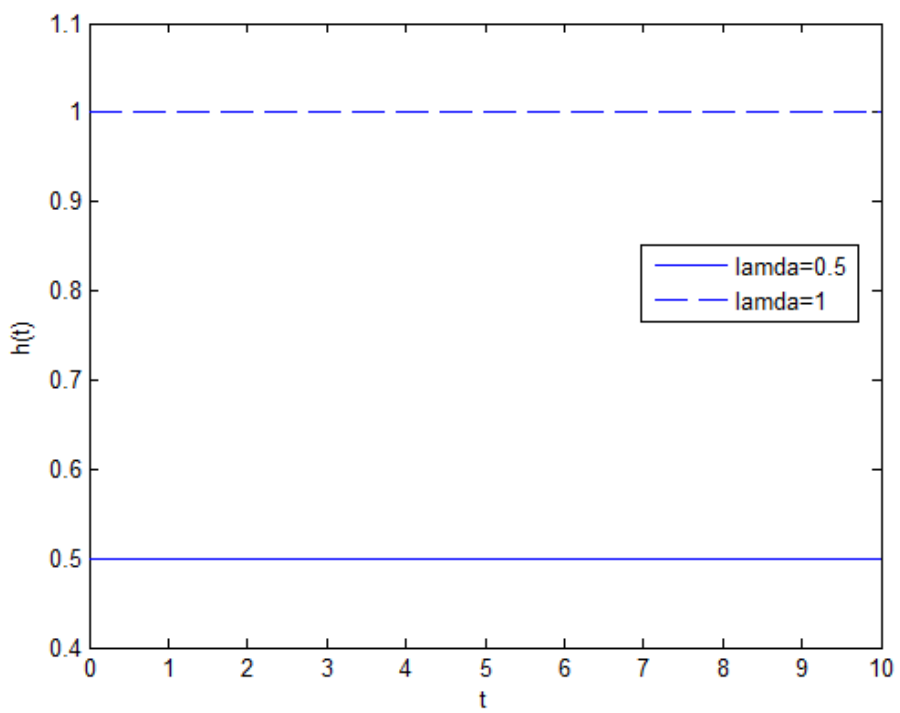

Figura 1.3: Tasa de falla de la distribución Exponencial, $\lambda=0.5,1$

\subsubsection{Distribución Weibull}

Esta distribución es utilizada para modelar tiempos de falla que presentan una tasa de falla que no es constante. Ahora ésta se define a partir de dos parámetros $\lambda$, 
que es el parámetro de escala y $\beta$, el parámetro de forma. Según sean los valores del parámetro $\beta$, esta distribución puede presentar tasas de falla crecientes, decrecientes o constantes. Por ejemplo:

- Cuando $\beta=1$, se tiene el caso del modelo exponencial, que tiene una tasa de falla constante.

- Cuando $\beta>1$, se tiene una tasa de falla creciente.

- Cuando $\beta<1$, se tiene una tasa de falla decreciente.

Función de densidad

$$
f(t)=\lambda \beta(\lambda t)^{\beta-1} \exp (-\lambda t)^{\beta}, \quad t \geq 0, \lambda>0, \beta>0
$$

\section{Función de distribución}

$$
F(t)=1-\exp (-\lambda t)^{\beta}
$$

\section{Función de confiabilidad}

$$
R(t)=\exp (-\lambda t)^{\beta}
$$

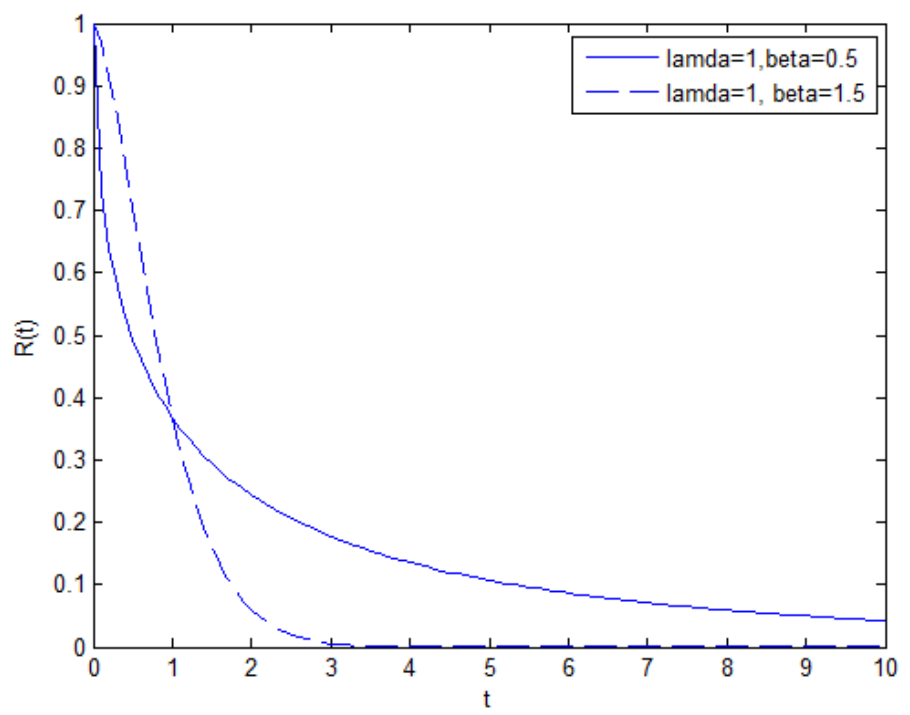

Figura 1.4: Función de confiabilidad de la distribución Weibull $\lambda=1, \beta=0.5,1.5$. 


\section{Tasa de falla}

$$
h(t)=\lambda \beta(\lambda t)^{\beta-1}
$$

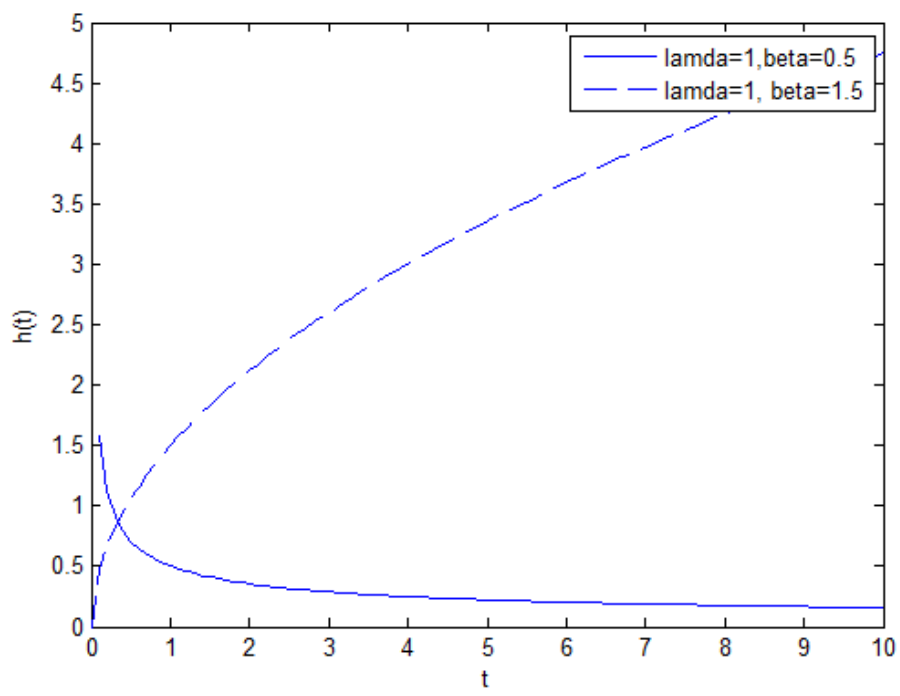

Figura 1.5: Tasa de falla de la distribución Weibull con $\lambda=1, \beta=0.5,1.5$.

\section{Función p-cuantil}

$$
t_{p}=\frac{1}{\lambda}(-\ln (1-p))^{\frac{1}{\beta}}
$$

\section{Tiempo medio entre falla (MTBF)}

$$
M T B F=\frac{1}{\lambda} \Gamma\left(1+\frac{1}{\beta}\right)
$$

donde $\Gamma(x)=\int_{0}^{\infty} t^{x-1} e^{-t} d t$. Se define $\Gamma(x)=(x-1) \Gamma(x-1)$ y para $n$ entero $\Gamma(n)=(n-1) !$

\subsubsection{Distribución Normal}

La distribución normal es una de las distribuciones más utilizadas, debido a que puede representar muchos fenómenos de la vida cotidiana. Esta distribución se define a partir de dos parámetros, la media $\mu$ que indica la tendencia central de un conjunto de datos, y la desviación estándar $\sigma$ que cuantifica el grado de dispersión de los datos 
respecto de la media.

En el área de ingeniería esta distribución es utilizada para construir Gráficas de Control de Procesos, las cuales fueron desarrollados por Shewhart (1931). Una gráfica de control es un gráfico en el cual se representan los valores de algún tipo de medición realizada durante el funcionamiento de un proceso continuo, para detectar causas no naturales de variación en el proceso. Se puede decir, que para todo proceso en el que sólo existe variación inherente o no causada los resultados siguen las características de una distribución normal. Es decir, aproximadamente el $67 \%$ de los resultados van a encontrarse dentro del intervalo $\pm 1 s$ donde $s$ es la desviación estándar muestral. Aproximadamente el $95 \%$ de los resultados se encuentran dentro del intervalo $\pm 2 s$ y aproximadamente el $99 \%$ de los resultados se encuentran dentro del intervalo $\pm 3 s$.

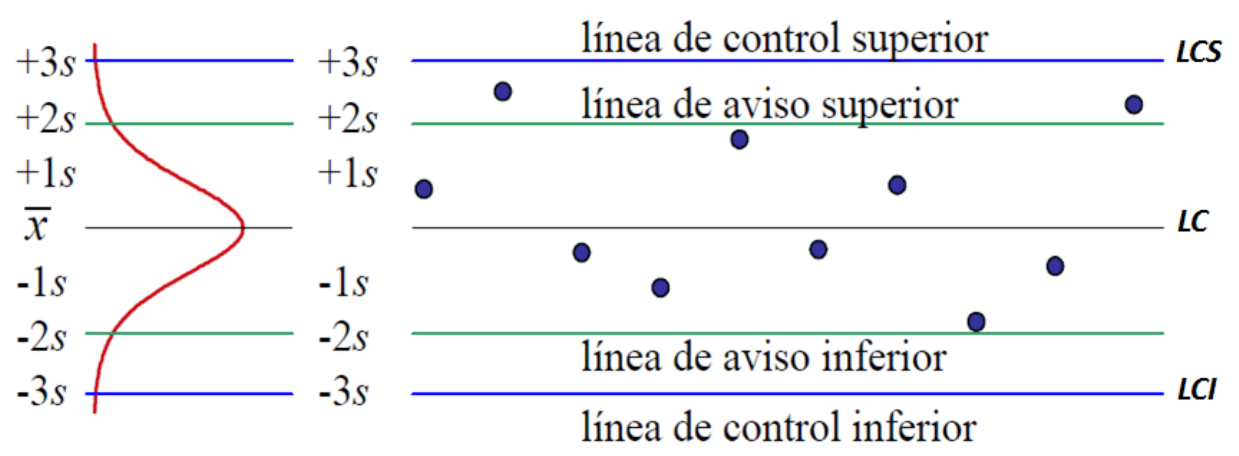

Figura 1.6: Límites control y su relación con la distribución normal.

La gráfica de control consiste en: una línea central $L C$ y dos pares de líneas límite espaciadas por encima y por debajo de la línea central, que se denominan Límite de Control Inferior LCI y Límite de Control Superior LCS. Éstos se establecen de tal manera que los valores situados entre los límites puedan atribuirse a la dispersión inherente del proceso, mientras que los que caigan fuera puedan interpretarse como una carencia de control del proceso. Si todos los puntos caen dentro de los límites de control, pero se observan tendencias, entonces también el proceso está fuera de control.

En el siguiente ejemplo se presenta la construcción de una gráfica de control para medias $\bar{X}$ y rangos $R$, el cual se utiliza para controlar los dos parámetros básicos de un proceso: la media y la variación. El rango $R$, se define como la diferencia entre los valores máximo y mínimo de un conjunto de datos. 
Para construir la gráfica se deben estimar la media y la desviación estándar. En la práctica el rango se utiliza como medida de dispersión.

Los límites de control, que se establecen a 3 desviaciones estándar, y la línea central son:

Para gráfica de medias:

- $L C I=\overline{\bar{X}}+A_{2} \bar{R}$

- $L C=\overline{\bar{X}}$

- $L C S=\overline{\bar{X}}-A_{2} \bar{R}$

Para la gráfica de rangos:

- $\mathrm{LCI}=D_{4} \bar{R}$

- $\mathrm{LC}=\bar{R}$

- $\mathrm{LCS}=D_{4} \bar{R}$

Donde $\overline{\bar{X}}=\sum_{i=1}^{n} \bar{X}_{i}, \bar{R}=\sum_{i=1}^{n} R_{i}$ y $n$ es el número de muestras. Los valores $A_{2}$ y $D_{4}$ [1] son constantes que dependen del tamaño de la muestra. Se recomienda utilizar un tamaño de muestra igual a 5 y un número de muestras no menor a 25.

Ejemplo 1.3.1. Una empresa de alimentos se dedica a la fabricación de mermeladas. La mermelada se vende en frascos de 200 gramos. El equipo de control de calidad supervisa el estado de control del proceso, para ello, se extraen cinco frascos de la línea de producción en intervalos de 10 minutos registrando el peso.

En el siguiente cuadro se muestran los valores obtenidos para la media y rango de cada una de las 25 muestras que se observaron. 


\begin{tabular}{|c|c|c|c|c|c|}
\hline No. muestra & $\bar{X}$ & $\bar{R}$ & No. muestra & $\bar{X}$ & $\bar{R}$ \\
\hline 1 & 200.80 & 6.00 & 14 & 208.80 & 10.00 \\
2 & 202.00 & 6.00 & 15 & 198.60 & 11.00 \\
3 & 203.00 & 7.00 & 17 & 206.40 & 4.00 \\
4 & 200.60 & 2.00 & 17 & 203.20 & 5.00 \\
5 & 200.20 & 11.00 & 18 & 200.80 & 8.00 \\
6 & 202.80 & 8.00 & 19 & 203.80 & 4.00 \\
7 & 200.40 & 4.00 & 20 & 204.80 & 6.00 \\
8 & 204.40 & 4.00 & 21 & 204.20 & 9.00 \\
9 & 203.40 & 6.00 & 22 & 205.60 & 2.00 \\
10 & 202.60 & 5.00 & 22 & 204.60 & 5.00 \\
11 & 201.00 & 4.00 & 24 & 203.60 & 5.00 \\
12 & 203.00 & 7.00 & 25 & 203.00 & 4.00 \\
13 & 203.60 & 1.00 & - & - & - \\
\hline
\end{tabular}

Para $n=5 A_{2}=0.58$ y $D_{4}=2.11$

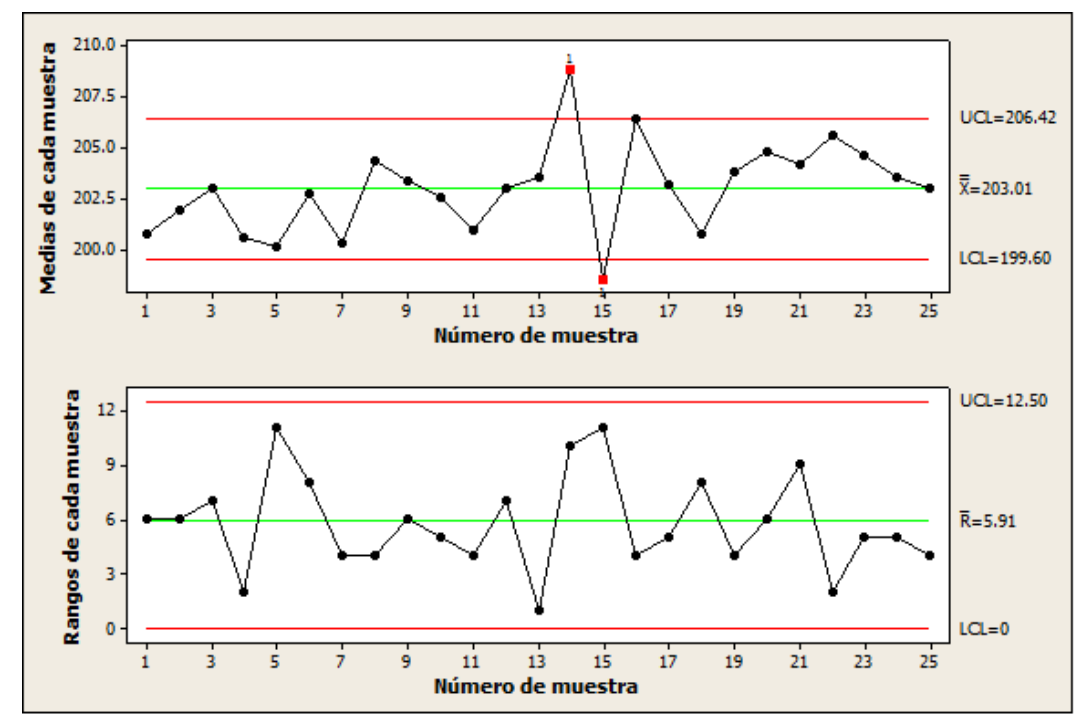

Figura 1.7: Gráfica de Control $\bar{X}-R$

En la Figura 1.7 se observan las gráficas de control tanto para medias como para los rangos. En la primera gráfica, la gráfica de medias, se puede observar que dos valores se encuentran fuera de los límites de control, uno por encima del límite superior y otro por debajo del límite inferior, los cuales corresponden a las muestras 14 y 15. Esto es indicio de que el proceso se encuentra fuera de control, es decir, que además de la variación natural, existe variación causada. En lo que se refiere a la gráfica de rangos, se puede observar que la variabilidad del proceso permanece 
estable, pues ninguno de los puntos de encuentra fuera de los límites de control.

En lo que sigue se presentan las diferentes funciones que caracterizan la distribución Normal.

\section{Función de densidad}

$$
f(t)=\frac{1}{\sigma \sqrt{2 \pi}} \exp \left(-\frac{1}{2}\left(\frac{t-\mu}{\sigma}\right)^{2}\right), \quad-\infty<t<\infty, \mu \geq 0, \sigma>0
$$

\section{Función de distribución}

$$
F(t)=\int_{-\infty}^{t} f(x) d x=\Phi\left(\frac{t-\mu}{\sigma}\right)
$$

Donde $\Phi$ es la función de distribución normal estándar, cuyos parámetro son $\mu=0$ y $\sigma=1$.

Función de confiabilidad

$$
R(t)=\int_{t}^{\infty} \frac{1}{\sigma \sqrt{2 \pi}} \exp \left(-\frac{1}{2}\left(\frac{t-\mu}{\sigma}\right)^{2}\right) d x=1-\Phi\left(\frac{t-\mu}{\sigma}\right)
$$

\section{Tiempo medio entre falla (MTBF)}

$$
M T B F=\mu
$$

\section{Función p-cuantil}

$$
t_{p}=\mu+\sigma \Phi^{-1}(p)
$$

donde $\Phi^{-1}$ es la función inversa de la distribución normal estándar acumulada. 


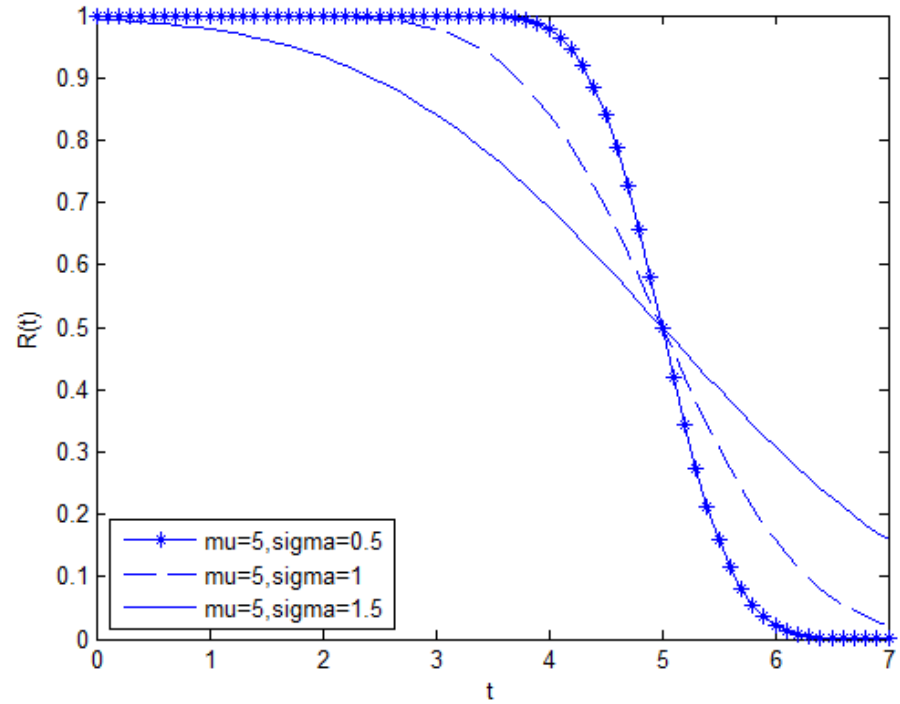

Figura 1.8: Función de confiabilidad de la distribución Normal, $\mu=5$, $\sigma=0.5,1,1.5$

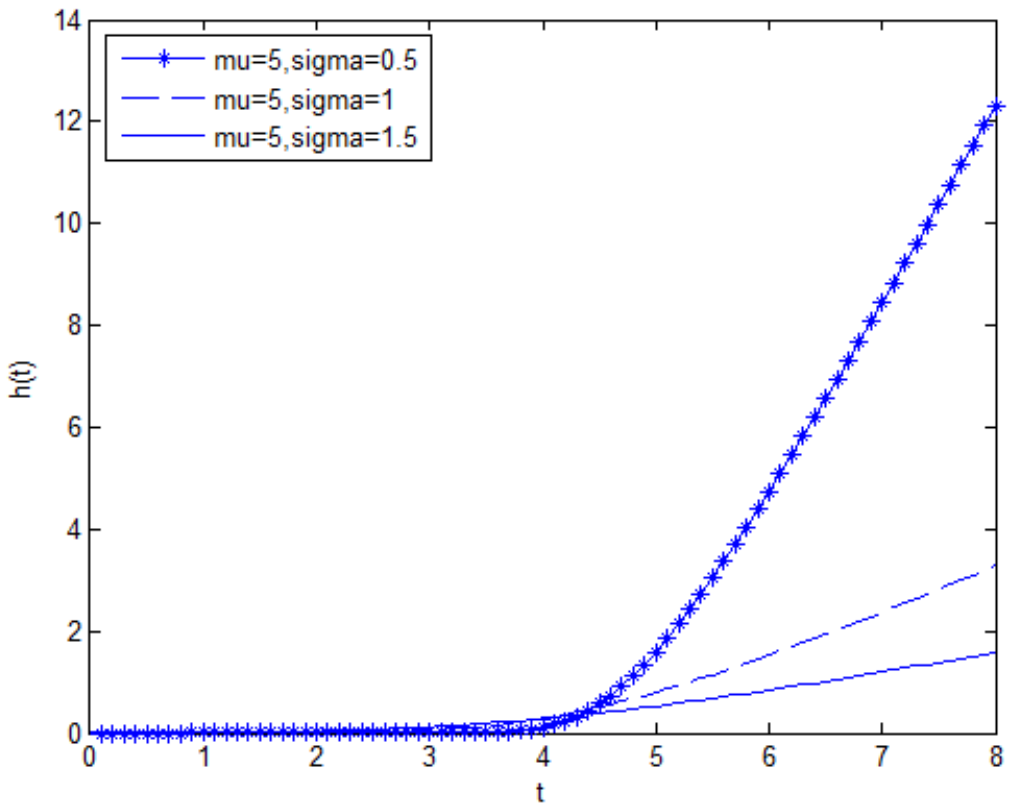

Figura 1.9: Tasa de falla de la distribución Normal, $\mu=5, \sigma=0.5,1,1.5$ 


\subsubsection{Distribución Lognormal}

La distribución Lognormal se relaciona con la distribución normal de la siguiente forma: si $X$ es una variable aleatoria normal con media $\mu$ y desviación estándar $\sigma$, entonces la variable aleatoria $Y=e^{X}$ tiene una distribución lognormal con parámetros $T_{50}=e^{\mu}$ y $\sigma$. Es decir, el logaritmo de una variable aleatoria lognormal cuyos parámetros son la mediana $T_{50}=e^{\mu}$ y $\sigma$, tiene distribución $N(\mu, \sigma)$. La variable aleatoria $T$ no asume valores iguales a cero ya que $\ln T$ no está definida para $T=0$.

La distribución Lognormal es útil es diversas áreas: en Confiabilidad se utiliza para modelar tiempos de reparación; en Medicina se utiliza para modelar el tiempo de supervivencia en pacientes con alguna enfermedad como cáncer y en Economía se usa para modelar la distribución personal de la renta y la distribución de ventas.

\section{Función de densidad}

$$
f(t)=\frac{1}{\sigma \sqrt{2 \pi}} \exp \left(-\frac{1}{2}\left(\frac{\ln t-\ln T_{50}}{\sigma}\right)\right)^{2}, \quad t>0, \mu \geq 0, \sigma>0
$$

Función de distribución

$$
F(t)=\int_{-\infty}^{t} f(x) d x=\Phi\left(\frac{\ln t-\ln T_{50}}{\sigma}\right)
$$

Función de confiabilidad

$$
R(t)=\int_{t}^{\infty} \frac{1}{\sigma \sqrt{2 \pi}} \exp \left(-\frac{1}{2} \frac{(x-\mu)^{2}}{\sigma}\right) d x=1-\Phi\left(\frac{\ln t-\ln T_{50}}{\sigma}\right)
$$

\section{Tiempo medio entre falla(MTBF)}

$$
M T B F=\exp \left(T_{50}+\frac{\sigma^{2}}{2}\right)=T_{50} \exp \left(\frac{\sigma^{2}}{2}\right)
$$




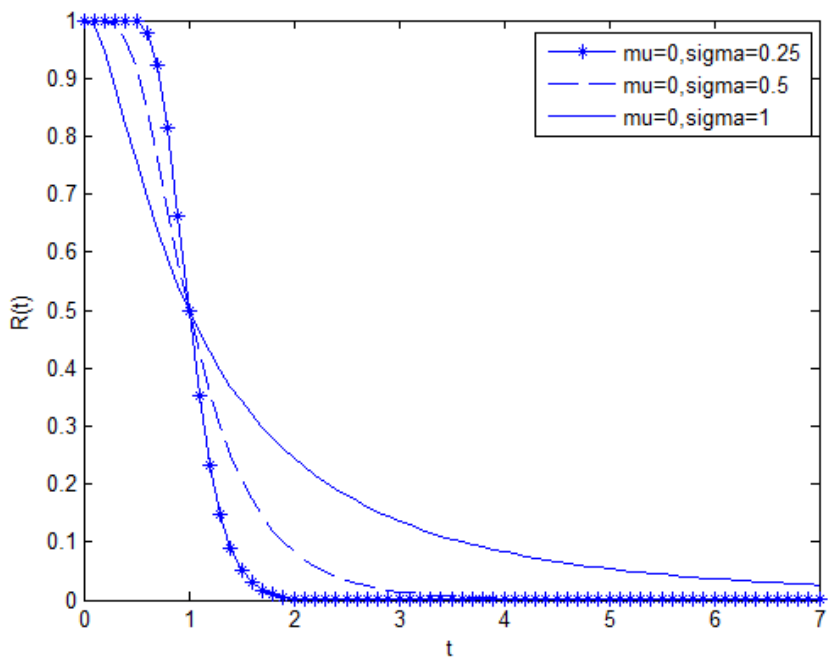

Figura 1.10: Función de confiabilidad de la distribución Lognormal, $\mu=0$, $\sigma=0.5,1,1.5$

\section{Función p-cuantil}

$$
t_{p}=T_{50} \exp \left(\sigma \Phi^{-1}(p)\right)
$$

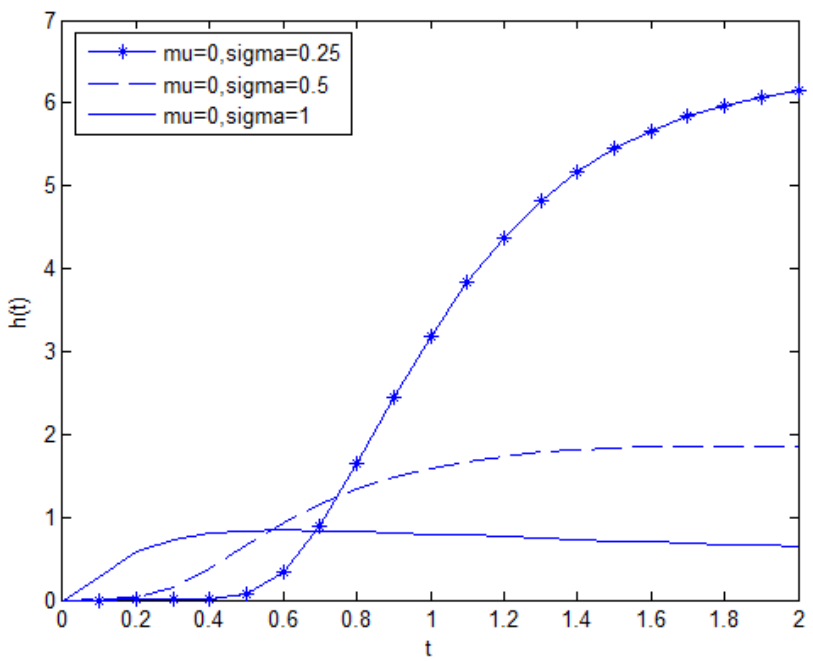

Figura 1.11: Tasa de falla de la distribución Lognormal, $\mu=0, \sigma=0.25,0.5,1$ 


\subsection{Censura de datos}

Con frecuencia ocurre que los datos asociados a tiempos de falla presentan observaciones incompletas, es decir, no se conocen los tiempos de falla de algunas de las unidades de manera exacta. Para el análisis de los datos se incluye toda la información de las observaciones, aún de aquellas que pudieron perderse durante el estudio o cuya falla sucede después de haber finalizado el estudio. Por esta razón, los datos se pueden clasificar en dos categorías: completos y censurados. Una observación en el tiempo $t$ es completa si representa el tiempo exacto en el que ocurrió la falla de la unidad. Si no se conocen los tiempos de falla de las unidades de manera exacta, sino sólo los intervalos de tiempo donde ocurrieron o pidieron haber ocurrido las fallas, se tienen datos censurados.

Existen tres posibles casos de datos censurados: censurados por la derecha, por la izquierda y por intervalo.

\section{Censura por la derecha.}

Se presenta cuando sabemos que la falla ocurre en algún punto posterior al tiempo de duración del estudio u observación.

Algunos tipos de censura por la derecha son:

- Censura tipo I (por tiempos). Se fija un tiempo determinado $C$ para la duración del estudio, y sobre ese intervalo de tiempo se observan las fallas ocurridas. En este caso, $C$ es una constante de censura prefijada por el analista para todas las unidades muestrales.

- Censura tipo II (por número de fallas). Se determina una cantidad $r<n$ de fallas con las cuales concluirá el estudio, $n$ es el número de unidades en observación.

Es importante señalar que los valores de censura $C$ y $r$ se fijan antes de iniciar el estudio y no durante el transcurso del mismo.

\section{Censura por la izquierda}

Este tipo de censura resulta cuando se sabe que el tiempo exacto de la falla de una unidad ocurrió antes de un cierto tiempo. Por ejemplo, se puede conocer que cierta unidad falló antes de las 100 horas pero no se conoce exactamente cuándo. En otras palabras, tal unidad podría haber fallado en algún tiempo entre 0 y 100 horas.

\section{Censura por intervalo}

Este tipo de censura ocurre cuando se sabe que el tiempo de falla ocurre dentro de un intervalo. Aquí el seguimiento de las unidades se realiza periódicamente y por tanto, 
la falla sólo puede conocerse entre dos periodos de revisión. Por ejemplo, supongamos que una unidad se inspecciona cada 100 horas; si se inspecciona a las 100 horas y encontramos que está operando y luego al realizar otra inspección a las 200 horas encontramos que ésta ya no se encuentra funcionando, entonces solamente se sabe que la unidad falló en el intervalo 100 y 200 horas.

\subsection{Estimación de parámetros por método de Máxima Verosimilitud (MV)}

Cuando se conoce la forma de la función de distribución del tiempo de falla es posible estimar los parámetros que la definen a partir de una muestra dada. Los estimadores más frecuentes son los de máxima verosimilitud. Un estimador de máxima verosimilitud del parámetro de una distribución se obtiene a partir de una muestra y es el valor del parámetro que da a la muestra la máxima probabilidad de ocurrencia.

Supóngase que $T_{1}, T_{2}, T_{3}, \ldots, T_{n}$ son variables aleatorias independientes e idénticamente distribuidas, las cuales representan los tiempos de falla $t_{1}, t_{2}, \ldots, t_{n}$ de $n$ unidades. Sean $f$ la función de densidad y $\theta=\left(\theta_{1}, \theta_{2}, \ldots, \theta_{n}\right)$ los parámetros a estimar. La función de verosimilitud se define como la función de probabilidad conjunta de las variables $T_{1}, T_{2}, T_{3}, \ldots, T_{n}$, evaluada en $t_{1}, t_{2}, \ldots, t_{n}$.

Si todas las observaciones $t_{i}$ son completas, la función de verosimilitud $L$ va a estar dada por el producto de las densidades individuales evaluadas en cada observación, como a continuación se indica:

$$
L(\theta)=\prod_{i: n} f\left(t_{i}\right)
$$

No obstante, hemos visto que las observaciones pueden estar incompletas, es decir, que pueden presentar algún tipo de censura. El caso más común es el de censura por la derecha, en este caso se supone que en el tiempo en el que se inician las observaciones, $t=0$, los componentes se encuentran funcionando .

Para poder caracterizar la función de verosimilitud para observaciones censuradas por la derecha, se define para cada unidad la función indicadora de censura en el tiempo $v_{i}$ como

$$
\delta_{i}= \begin{cases}1, & \text { si } t_{i} \leq v_{i} \\ 0, & \text { si } t_{i}>v_{i}\end{cases}
$$


Es decir, si $t_{i}$ es una observación de tiempo de falla, $\delta_{i}=1$, y es una observación censurada si $\delta_{i}=0$ y en este caso se toma $t_{i}=v_{i}$ como el último momento en que la unidad fue observada y seguía funcionando. De esta forma, se tiene que las observaciones estarán representadas por la parejas $\left(t_{i}, \delta_{i}\right)$.

A partir de lo anterior, la función de verosimilitud para observaciones censuradas por la derecha va a estar dada por

$$
L(\theta)=\prod_{i: n} f\left(t_{i}\right)^{\delta_{i}} R\left(t_{i}\right)^{1-\delta_{i}}
$$

Entonces, si la observación es completa contribuye a la función de verosimilitud con su función de densidad, y si es censurada, contribuye con su función de confiabilidad. Cuando las observaciones son censuradas por la izquierda la función de densidad se reemplaza por la función de distribución y el caso en el que existen observaciones censuradas por intervalo, la función de densidad se sustituye por la probabilidad de falla en ese intervalo.

Finalmente, las estimaciones de los parámetros de la distribución se obtienen maximizando la función $L(\theta)$ [3].

\subsubsection{Estimación del parámetro de la distribución Ex- ponencial}

Supongamos que se tienen $t_{1}, t_{2}, t_{3}, \ldots, t_{n}$ observaciones independientes de tiempos de falla con distribución exponencial, correspondientes a $n$ componentes puestos a prueba. Supongamos además que de las $n$ observaciones, $n-r$ son observaciones censuradas por la derecha.

Recordemos que la función de densidad de la distribución Exponencial está dada por $f(t)=\lambda \exp (-\lambda t)$, siendo $\lambda$ el parámetro a estimar. Dado que existen observaciones censuradas por la derecha, la función de verosimilitud queda expresada como:

$$
L(\lambda)=\prod_{i}^{n} f\left(t_{i}\right)^{\delta_{i}} R(T)^{1-\delta_{i}}=\prod_{i}^{n}\left(\lambda \exp \left(-\lambda t_{i}\right)\right)^{\delta_{i}}\left(\exp \left(-\lambda t_{i}\right)\right)^{1-\delta_{i}}
$$


Si el valor de censura es el mismo para las $n$ unidades, digamos $v$ y las primeras $r$ observaciones son completas y las últimas $n-r$ son censuradas en $v$ entonces

$$
r=\sum_{i=1}^{r} 1
$$

Luego

$$
L(\lambda)=\prod_{i}^{r} \lambda \exp \left(-\lambda t_{i}\right)(\exp (-\lambda v))^{n-r}
$$

Para hacer los cálculos más sencillos, tomamos el logaritmo natural de la función de verosimilitud $L(\lambda)$. Recordemos que el logaritmo es una función monótona creciente, así que, el máximo de $L(\lambda)$ y de $\ln (L(\lambda))$ se alcanzan en el mismo punto.

$$
\ln (L(\lambda))=r \ln \lambda-\lambda \sum_{i=1}^{r} t_{i}-(n-r)(\lambda v)
$$

Si derivamos respecto a $\lambda$ obtenemos

$$
\frac{\partial}{\partial \lambda} \ln (L(\lambda))=\frac{r}{\lambda}-\sum_{i=1}^{r} t_{i}-(n-r) v
$$

Finalmente si igualamos la derivada a cero tenemos

$$
\frac{r}{\lambda}=\sum_{i=1}^{r} t_{i}-(n-r) v
$$

Por lo que un estimador para el parámetro $\lambda$ es

$$
\hat{\lambda}=\frac{r}{\sum_{i=1}^{r} t_{i}-(n-r) v} .
$$

En esta última ecuación, si consideramos censura de tipo I, $t_{1}, t_{2}, \ldots, t_{r}$ son los tiempos de falla de las $r$ unidades que fallaron antes de que finalizara la prueba, en el tiempo $v$. Por otro lado, si consideramos censura de tipo II, es decir, que la prueba termina hasta que se presenta el r-ésimo tiempo de falla, entonces $v=t_{r} \mathrm{y}$ el estimador queda expresado como:

$$
\hat{\lambda}=\frac{r}{\sum_{i=1}^{r} t_{i}-(n-r) t_{r}}
$$




\subsection{Estimación no paramétrica}

Ahora se abordarán algunas técnicas no paramétricas que permitirán estimar ya sea la función de distribución $F(t)$ o alternativamente la función de confiabilidad $R(t)=1-F(t))$ asociada a las observaciones, tanto para observaciones completas como para observaciones censuradas.

\subsubsection{Estimación de la función de distribución en caso de observaciones completas}

Primero vamos a presentar la función que permite estimar la función de distribución para el caso en que ninguna de las observaciones es censurada.

Sea $T$ una variable aleatoria que representa el tiempo a la falla. Considérese una muestra de $n$ dispositivos de los cuales se han observado los tiempos de falla $t_{1}, t_{2}, \ldots, t_{n}$. A partir de los estadísticos de orden de la muestra $t_{(1)}<t_{(2)}<\ldots<t_{(n)}$ se define la función de distribución empírica de $F$ como:

$$
\hat{F}(t)= \begin{cases}0, & \text { si } 0<t<t_{(1)} \\ \frac{i}{n}, & \text { si } t_{(i)} \leq t<t_{(i+1)}, i=1,2, \ldots, n-1 \\ 1, & \text { si } t_{(n)} \leq t\end{cases}
$$

A partir de la expresión anterior podemos ver que el estimador para la función de distribución $\hat{F}(t)$ se caracteriza por

$$
\hat{F}(t)=\frac{\text { num.observaciones } \leq t}{n}
$$

Es decir, $\hat{F}(t)$ representa la fracción de dispositivos que han fallado hasta antes del instante t. Una vez que se ha estimado la función $F(t)$ podemos obtener el estimador para $R(t): \hat{R}(t)=1-\hat{F(t)}$.

Ejemplo 1.6.1. Se realizó una prueba de vida sobre diez dispositivos idénticos, y se obtuvieron los siguientes tiempos de falla: 89, 132, 202, 263, 321, 362, 421, 473, 575 y 633 horas. Se obtendrá el estimador no paramétrico de la función de distribución 
para $t=350$ horas.

\section{Solución:}

Usando la expresión de la función de distribución empírica, y puesto que en este caso $n=10$ y hay sólo 5 observaciones cuyo tiempo de falla es inferior o igual a 350 horas, se tiene que $\hat{F}(350)=\frac{5}{10}=\frac{1}{2}$

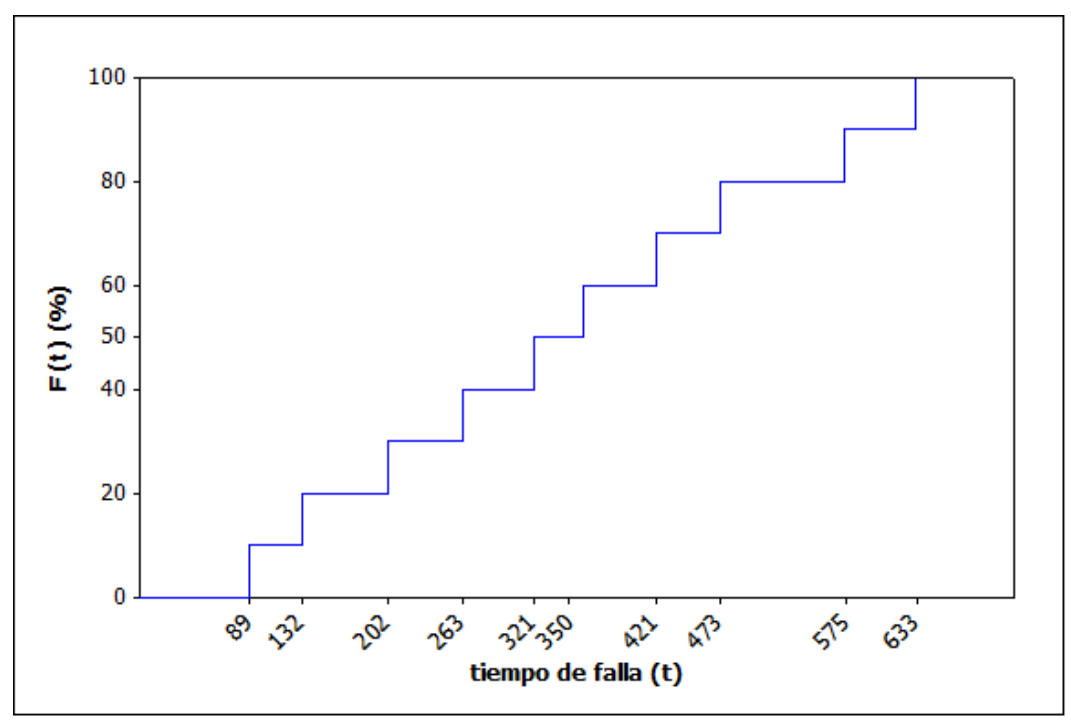

Figura 1.12: Función de distribución empírica

En la Figura 1.12 se muestra la representación gráfica de la función de distribución empírica de los tiempos de falla. Observe que la gráfica es una función escalonada creciente con saltos discontinuos en cada uno de los tiempos de falla, y dado que todos éstos son distintos, la función crece en proporción $\frac{1}{10}$ en cada uno. Note también que $\hat{F}(t)=0$ para los valores de $t$ anteriores a la primera falla y $F(t)=1$ después del último tiempo de falla.

\subsubsection{Estimación de la función de confiabilidad en ca- so de observaciones censuradas.}

Ahora vamos a presentar el estimador de Kaplan Meier, el cual permite obtener un estimador no paramétrico para la función de confiabilidad cuando las observaciones presentan algún tipo de censura. Se define el estimador de Kaplan Meier para 
el caso en que se tienen observaciones censuradas por la derecha.

Sea $T$ una variable aleatoria que representa el tiempo a la falla de una unidad. Considérese una muestra formada por $n$ unidades de las cuales se observan $t_{1}, t_{2}, \ldots, t_{n}$ tiempos de falla (incluyendo censuras) y sean $t_{(1)}<t_{(2)}<\ldots<t_{(k)}$ los estadísticos de orden de los $k$ tiempos de falla distintos que fueron observados.

Sean $n_{i}$ el número de unidades bajo observación que están funcionando antes del instante $t_{i}, d_{i}$ el número de dispositivos que fallan justo antes del instante $t_{i}$ y $c_{i}$ el número de censuras que ocurren en el intervalo $\left[t_{i}, t_{i+1}\right)$. Se puede ver que $n_{0}=0$, $t_{0}=0, d_{0}=0$ y $n_{i+1}=n_{i}-d_{i}-c_{i}, \quad i=1,2, \ldots, k-1$.

Se define el estimador de Kaplan Meier para la función de confiabilidad $R(t)$ como

$$
\hat{R}(t)=\prod_{i: t_{(i)} \leq t}\left(1-\frac{d_{i}}{n_{i}}\right)
$$

Ejemplo 1.6.2. Se realizó una prueba de vida con 19 unidades idénticas y se obtuvieron los siguientes tiempos a la falla. Los tiempos censurados están marcados con un asterisco *

$5,5,6,6^{*}, 7,8^{*}, 8,9^{*}, 9^{*}, 12,13,13^{*}, 15^{*}, 16^{*}, 17,24,25,27^{*}, 30^{*}$

Vamos a calcular el estimador de Kaplan-Meier para estimar la función de confiabilidad $R(t)$.

Solución:

En este caso, se tienen 10 observaciones completas y 9 observaciones censuradas. Como son 10 tiempos de falla distintos, se consideran nueve intervalos durante los cuales ocurrieron las fallas. En la siguiente tabla, se muestra un resumen de los cálculos realizados para obtener el estimador de Kaplan-Meier para $R(t)$. 


\begin{tabular}{|c|c|c|c|c|c|}
\hline$i$ & {$\left[t_{i}, t_{i+1}\right)$} & $n_{i}$ & $d_{i}$ & $1-\frac{d_{i}}{n_{i}}$ & $\widehat{R}(t)$ \\
\hline 0 & {$[0,5)$} & 19 & - & - & 1 \\
1 & {$[5,6)$} & 19 & 2 & 0.894737 & 0.894737 \\
2 & {$[6,7)$} & 17 & 1 & 0.941176 & 0.842105 \\
3 & {$[7,8)$} & 15 & 1 & 0.933333 & 0.785965 \\
4 & {$[8,12)$} & 14 & 1 & 0.928571 & 0.729825 \\
5 & {$[12,13)$} & 10 & 1 & 0.9 & 0.656842 \\
6 & {$[13,17)$} & 9 & 1 & 0.88888 & 0.583860 \\
7 & {$[17,24)$} & 5 & 1 & 0.8 & 0.467088 \\
8 & {$[24,25)$} & 4 & 1 & 0.75 & 0.350316 \\
9 & {$[25,+\infty)$} & 3 & 1 & 0.66666 & 0.233544 \\
\hline
\end{tabular}

Note que en el intervalo $[0,5)$ se observan las 19 unidades y ninguna falla antes de 5 , en el intervalo $[5,6)$ se observan nuevamente las 19 unidades y ahora fallan 2 en 5 , en el intervalo $[6,7)$ se observan 17 unidades, hay una falla en 6 y una unidad censurada que afecta sólo al número de unidades observadas.

En la Figura 1.13 se muestra la representación gráfica de la estimación no paramétrica para la función de confiabilidad $R(t)$. Observe que esta representación es una función escalonada que decrece. Cada discontinuidad se produce en los instantes donde hay fallos y la altura de esta discontinuidad es 1 menos la proporción de fallos en ese instante.

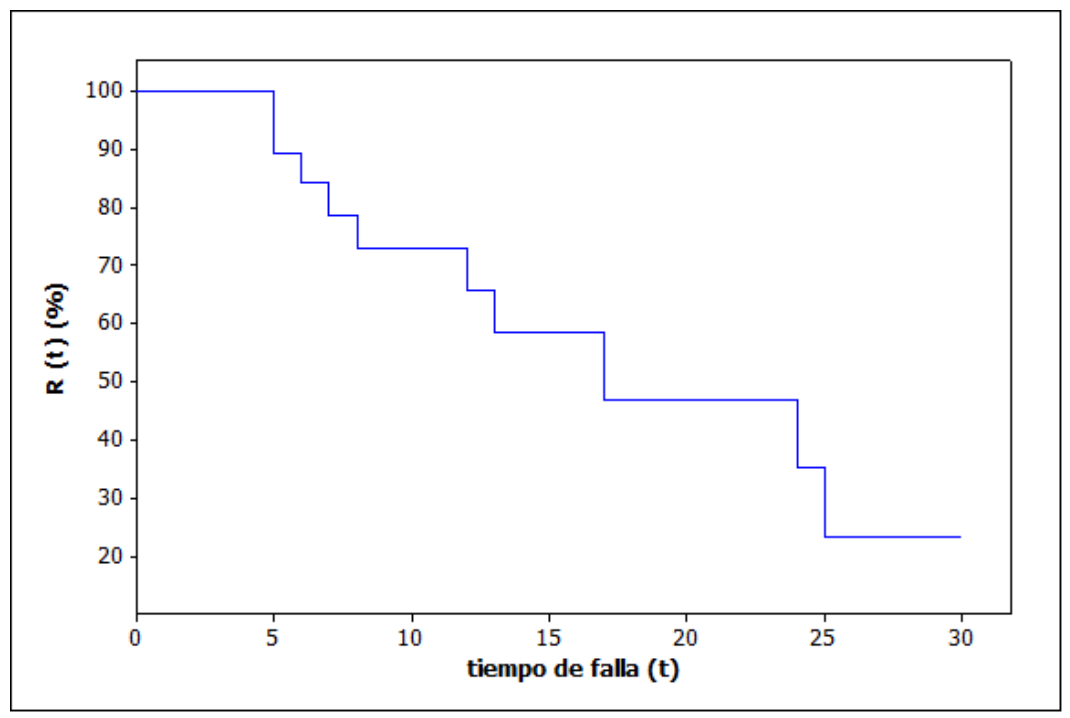

Figura 1.13: Estimación no paramétrica de $R(t)$ 


\subsection{Estimación de parámetros mediante linealización de la función de distribución.}

En esta sección veremos como realizar la estimación de los parámetros de las distribuciones de tiempos de falla, linealizando la función de distribución y luego resolviendo con regresión lineal.

Primero vamos a linealizar el modelo de distribución, para enseguida mostrar en un gráfico la línea recta que describe este modelo junto con el conjunto de observaciones, que pueden ser completas o censuradas. En este gráfico vamos a juzgar el ajuste de las observaciones a la línea recta. Si el ajuste es bueno podemos concluir que las observaciones se caracterizan por tener dicha distribución. Finalmente, las estimaciones de los parámetros de la distribución se obtienen a partir de los coeficientes de la ecuación de línea recta: pendiente y ordenada al origen.

Enseguida se muestran las ecuaciones linealizadas para cada uno de lo modelos que se mencionaron anteriormente. Éstas están dadas en la forma $y=a x+b$.

\section{Distribución Exponencial}

Para esta distribución la ecuación linealizada, a partir de la función de distribución, se escribe como:

$$
-\ln (1-(F(t)))=\lambda t
$$

En este caso el valor estimado del parámetro $\lambda$ de la distribución exponencial, estará dado por la pendiente de la recta que pasa por el origen, es decir, $\hat{\lambda}=a$.

\section{Distribución Weibull}

De la función de distribución Weibull la ecuación linealizada resultantes es

$$
\ln (-\ln (1-(F(t))))=\beta \ln t+\beta \ln (\lambda)
$$

Para esta distribución, las estimaciones de cada uno de sus dos parámetros serán

$$
\hat{\beta}=a
$$


$\mathrm{y}$

$$
\hat{\lambda}=e^{\frac{b}{\hat{\beta}}}
$$

\section{Distribución Normal}

Para la distribución Normal se obtiene la siguiente ecuación

$$
\Phi^{-1}(F(t))=\frac{t}{\sigma}-\frac{\mu}{\sigma}
$$

Los valores de los parámetros estimados son $\hat{\sigma}=\frac{1}{a}$ y $\hat{\mu}=-b \hat{\sigma}$.

\section{Distribución Lognormal}

La ecuación linealizada que se obtiene para esta distribución es

$$
\Phi^{-1}(F(t))=\frac{\ln t}{\sigma}-\frac{\mu}{\sigma}
$$

Aquí nuevamente $\hat{\sigma}=\frac{1}{a}$ y $\hat{\mu}=-b \hat{\sigma}$.

Hasta este momento sólo conocemos la función de distribución empírica, la cual nos permite estimar $F(t)$; sin embargo, en la práctica se utilizan algunas otras definiciones, las más usada debido a los buenos resultados que presenta es

$$
\hat{F}(t)=\frac{i-0.3}{n+0.4}
$$

Esta fórmula es conocida como el estimador de Bernard [4],5] donde $i$ es el número de fallas que han ocurrido antes o en el instante $t$. No se debe olvidar que los tiempos de falla deben estar ordenados.

El siguiente ejemplo ilustra el uso del estimador de Bernard para estimar la función de distribución. 
Ejemplo 1.7.1. Considérense 5 modelos de camiones idénticos comprados en las mismas fechas para realizar la mismas operaciones. Después de 3 meses de operación se realiza el análisis de fallas de los camiones. Se encontró que ciertas correas $\square^{1}$ de alguna toma de fuerza del sistema de diesel han experimentado un importante número de fracasos. Después de una investigación se obtuvo la información correspondiente al tiempo de falla de las correas, contando a partir de $t=0$. Ver Cuadro 1.1

Cuadro 1.1: Periodo de falla

\begin{tabular}{|c|c|c|c|c|c|}
\hline Período (mes) & Camión 1 & Camión 2 & Camión 3 & Camión 4 & Camión 5 \\
\hline 1 & - & Falla & - & Falla & - \\
2 & - & - & Falla & Falla & - \\
3 & - & - & - & - & Falla \\
\hline
\end{tabular}

A partir del método de linealización vamos a estimar los parámetros de la distribución Weibull, para modelar el tiempo de falla de las correas.

Se sabe que cada camión trabaja aproximadamente 300 horas por mes. El registro termina al final del tercer mes (hay censura tipo I, pues el final de la prueba se fija en $t=900$ hrs.). Se supone que cada vez que falla una correa, ésta es reemplazada por una nueva y la toma de fuerza funciona como nueva.

En el Cuadro 1.2 se muestran los tiempos de operación de cada correa antes de que ésta falle. Entre paréntesis se ubica el tiempo de operación en caso de que la correa haya sido reemplazada anteriormente.

Cuadro 1.2: Tiempos de falla (horas) de las correas.

\begin{tabular}{|c|c|c|c|c|c|}
\hline Período (mes) & Camión 1 & Camión 2 & Camión 3 & Camión 4 & Camión 5 \\
\hline 1 & - & 100 & - & 250 & - \\
2 & - & - & 350 & $450(200)$ & - \\
3 & - & - & - & - & 850 \\
\hline
\end{tabular}

En Cuadro 1.3 se muestra el número de horas de operación que lleva la correa de cada camión al final de la prueba ( $t=900 \mathrm{hrs})$.

Ahora vamos a obtener una aproximación de la función de distribución $F(t)$ con los tiempos de falla mostrados en el Cuadro 1.2. Para ello utilizamos el estimador de Bernard (Ecuación 1.14), para el cual se requiere que los tiempos de operación estén

\footnotetext{
${ }^{1}$ Una correa es una transmisión flexible que se usa para generar movimiento o transmitir potencia. Las correas se conocen también como bandas.
} 
Cuadro 1.3: Tiempo de operación (horas) de las correas al finalizar la prueba.

\begin{tabular}{|c|c|c|c|c|}
\hline Camión 1 & Camión 2 & Camión 3 & Camión 4 & Camión 5 \\
\hline 900 & 800 & 550 & 450 & 50 \\
\hline
\end{tabular}

ordenados de forma creciente como en el Cuadro 1.4. Se tienen 5 camiones; cada uno representa una muestra, entonces $n=5$. Note que la segunda falla del camión 4 ocurre en el tiempo $t=450$, pero ésta llevaba 200 hrs. funcionando.

Cuadro 1.4: Tiempos de falla ordenado en orden creciente.

\begin{tabular}{|c|c|c|}
\hline Orden de la falla & Camión & Tiempo de operación \\
\hline 1 & 2 & 100 \\
2 & 4 & 200 \\
3 & 4 & 250 \\
4 & 3 & 350 \\
5 & 5 & 850 \\
\hline
\end{tabular}

En el Cuadro 1.5 se muestran los valores de la estimación para $F(t)$.

Cuadro 1.5: Estimación para $F(t)$ utilizando datos completos sin censura.

\begin{tabular}{|c|c|}
\hline$t_{i}$ & $\widehat{F}_{i}$ \\
\hline 100 & 0.13 \\
200 & 0.31 \\
250 & 0.5 \\
350 & 0.69 \\
850 & 0.87 \\
\hline
\end{tabular}

En la segunda parte, además de considerar los tiempos de falla del Cuadro 1.2, vamos a considerar los tiempos de falla que resultan de la censura al final del tercer mes, los cuales se presentan el el Cuadro 1.3. A partir de esta estimación vamos a estimar los parámetros de la distribución Weibull.

El objetivo de incluir los datos censurados en el análisis es obtener una mejor estimación de la función de distribución, pues de esta forma evitamos subestimar la función de confiabilidad. El subestimar la confiabilidad de las correas nos llevaría a pensar que los autobuses requieren de un mantenimiento innecesario.

En el Cuadro 1.6 se muestran todos los tiempos de falla de las correas de cada camión. Si al finalizar la prueba, la correa seguía funcionando, el tiempo de falla registrado para ésta se considera como un dato censurado. 
Cuadro 1.6: Tiempos de falla (horas), considerando datos censurados

\begin{tabular}{|c|c|c|c|c|}
\hline$i$ & Falla-F, Censurada-C & Hrs. de operación & Camión & No. de correa \\
\hline 1 & $\mathrm{C}$ & 50 & 5 & 2 \\
2 & $\mathrm{~F}$ & 100 & 2 & 1 \\
3 & $\mathrm{~F}$ & 200 & 4 & 2 \\
4 & $\mathrm{~F}$ & 250 & 4 & 1 \\
5 & $\mathrm{~F}$ & 350 & 3 & 1 \\
6 & $\mathrm{C}$ & 450 & 4 & 3 \\
7 & $\mathrm{C}$ & 550 & 3 & 2 \\
8 & $\mathrm{C}$ & 800 & 2 & 2 \\
9 & $\mathrm{~F}$ & 850 & 5 & 1 \\
10 & $\mathrm{C}$ & 900 & 1 & 1 \\
\hline
\end{tabular}

Como se tienen datos censurados vamos a utilizar el estimador de Kaplan Meier (Ecuación 1.8) para obtener la estimación de la función de confiabilidad, $\tilde{R}(t)$, a partir de la cual podemos obtener la nueva estimación de la función de distribución, $\tilde{F}(t)$. En el Cuadro 1.7 se muestran los resultados obtenidos para cada estimación.

Cuadro 1.7: Estimación para $F(t)$ incluyendo datos censurados

\begin{tabular}{|c|c|c|}
\hline$i$ & $\tilde{R}_{i}$ & $\tilde{F}_{i}$ \\
\hline 100 & 0.89 & 0.11 \\
200 & 0.78 & 0.22 \\
250 & 0.67 & 0.33 \\
350 & 0.56 & 0.44 \\
850 & 0.28 & 0.72 \\
\hline
\end{tabular}

Para terminar, procedemos a calcular los parámetros de la distribución Weibull haciendo uso de esta última estimación para $F(t)$. En la Ecuación (1.10), la cual representa la ecuación linealizada para la distribución Weibull hacemos

$$
x=\ln t \quad y \quad y=\ln (-\ln (1-(\tilde{F}(t))))
$$

Utilizamos el método de minimos cuadrados para encontrar la ecuación de la línea recta que mejor se ajuste a las parejas de puntos $(x, y)$. La ecuación de la recta ajustada es $y=-7.30+1.13 x$. Finalmente por Ecuaciones (1.11) y (1.13) los estimadores de los parámetros de la distribución Weibull son

$$
\widehat{\beta}=1.13 \quad y \quad \widehat{\lambda}=627.96
$$




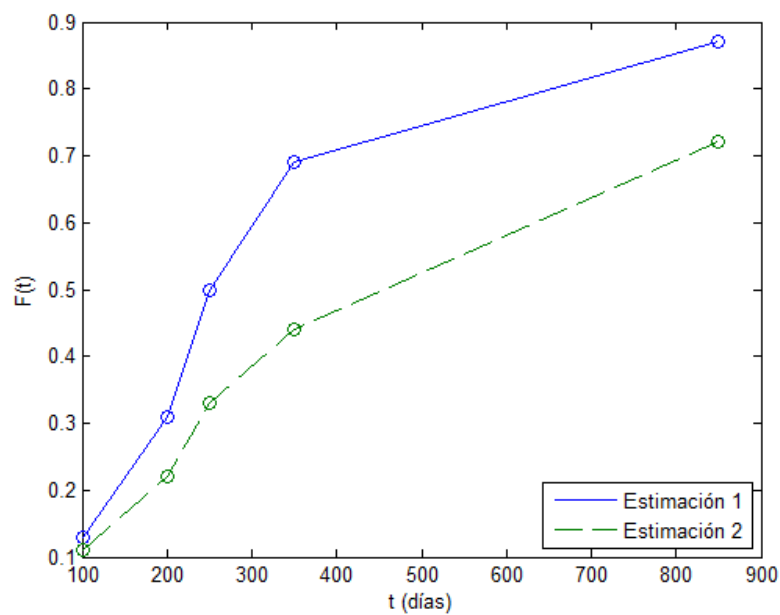

Figura 1.14: Estimaciones de la función de distribución acumulada.

\subsection{Modelos de vida acelerada}

\subsubsection{Distribución del tiempo de falla, bajo vida acelerada}

Cuando se quiere medir la confiabilidad de un componente es necesario tener una gran cantidad de información correspondiente a los tiempos de falla del componente, y se requiere que esta información esté disponible en un corto espacio de tiempo.

Para poder obtener esta información de manera más rápida se utilizan pruebas de vida acelerada en las cuales los componentes son sometidos a condiciones de operación más extremas de lo normal (llamadas también condiciones de estrés), con lo que se conseguirá acelerar el proceso de desgaste y, en consecuencia, se reducirá la duración del estudio. Los resultados así obtenidos sobre los tiempos de falla se podrán extrapolar al caso en el que los dispositivos se encuentran operando en condiciones normales.

Normalmente lo que se hace es incrementar alguna de las variables aceleradoras del componente o unidad (temperatura, voltaje, presión, humedad, etc.), esto causa tiempos de falla menores a los tiempos de fallo en condiciones normales. 
Una suposición importante que se hace en estos modelos es que cuando el componente se encuentra operando en condiciones de estrés, tendrá el mismo mecanismo de falla que cuando se encuentra operando en condiciones normales, con la única diferencia de que la falla aparece más rápido.

El incremento de la variable aceleradora puede ser constante o escalonado. En la práctica el método más usado es el de aceleración constante. En este método cada unidad o componente se somete a esfuerzo constante durante el estudio.

Cuando se supone aceleración constante se tiene la siguiente relación para los tiempos de falla $t_{o}=\epsilon t_{\epsilon}$, donde $\epsilon$ es el factor de aceleración constante, $t_{\epsilon}$ es el tiempo de falla bajo condiciones de estrés y $t_{o}$ es el tiempo de falla en condiciones normales.

La función de distribución acumulada del tiempo de falla en condiciones normales $F_{o}\left(t_{o}\right)$ para un cierto tiempo $t_{o}$ está dada por

$$
\begin{aligned}
F_{o}\left(t_{o}\right) & =P\left(T_{o}<t_{o}\right) \\
& =P\left(\frac{T_{o}}{\epsilon}<\frac{t_{o}}{\epsilon}\right) \\
& =P\left(T_{\epsilon}<t_{\epsilon}\right) \\
& =F_{\epsilon}\left(\frac{t_{o}}{\epsilon}\right)
\end{aligned}
$$

De modo que $F_{o}\left(t_{o}\right)=F_{\epsilon}\left(\frac{t_{o}}{\epsilon}\right)$.

La función de densidad en condiciones normales de operación para un cierto instantes $t$ es

$$
\begin{aligned}
f_{o}(t) & =\frac{d}{d t} F_{\epsilon}\left(\frac{t}{\epsilon}\right) \\
& =\frac{1}{\epsilon} f_{\epsilon}\left(\frac{t}{\epsilon}\right)
\end{aligned}
$$

La función de confiabilidad en condiciones normales de operación para un cierto tiempo $t$ es

$$
R_{o}(t)=1-F_{o}(t)=1-F_{\epsilon}\left(\frac{t}{\epsilon}\right)=R\left(\frac{t}{\epsilon}\right)
$$

La tasa de falla bajo condiciones normales de operación para un cierto tiempo $t$ 
es

$$
\begin{aligned}
h_{o}(t) & =\frac{f_{o}(t)}{R_{o}(t)} \\
& =\frac{\frac{1}{\epsilon} f_{\epsilon}\left(\frac{t}{\epsilon}\right)}{R_{\epsilon}\left(\frac{t}{\epsilon}\right)} \\
& =\frac{1}{\epsilon} h_{\epsilon}\left(\frac{t}{\epsilon}\right)
\end{aligned}
$$

Enseguida se muestra la función de confiabilidad de los modelos de distribución de confiabilidad bajo condiciones de operación normales, a partir de la estimación bajo condiciones aceleradas con factor de aceleración $\epsilon$.

- Distribución Weibull

$$
R(t)=e^{-\left(\frac{\lambda}{\epsilon} t\right)^{\beta}}
$$

- Distribución exponencial

$$
R(t)=e^{-\left(\frac{\lambda}{\epsilon} t\right)}
$$

- Distribución Normal

$$
R(t)=1-\Phi\left(\frac{t-\epsilon \mu}{\epsilon \sigma}\right)
$$

- Distribución Lognormal

$$
R(t)=1-\Phi\left(\frac{\ln t-\ln \epsilon-\mu}{\sigma}\right)
$$

Ejemplo 1.8.1. Un componente se probó en $180^{\circ} \mathrm{F}$, se encontró que el tiempo de falla sigue una distribución Weibull con parámetro de forma $\beta=3.5$ y parámetro de escala $\lambda=\frac{1}{19.3}$. La temperatura con la cual opera el componente en condiciones normales es $110^{\circ} \mathrm{F}$ y el factor de aceleración entre estas dos temperaturas es 12. Determinaremos la confiabilidad del componente bajo condiciones de operación normales en $t=200$.

Sabemos que la confiabilidad del componente en cualquier instante de tiempo está dada por

$$
R(t)=\exp \left[-\left(\frac{t}{12(19.3)}\right)^{3.5}\right]
$$


Entonces en $t=200$ la confiabilidad del componente es

$$
R(t)=\exp \left[-\left(\frac{200}{12(19.3)}\right)^{3.5}\right]=0.550
$$

Para determinar el factor de aceleración se utilizan los modelos de aceleración, que usan datos de falla que fueron obtenidos en condiciones aceleradas (o de estrés). Éstos suponen que el tiempo de falla es una función del estrés aplicado y otros valores constantes. Como los tiempos de falla son completamente aleatorios, un modelo de aceleración se interpreta como una ecuación que calcula el parámetro de escala de una distribución o cualquier $p$-cuantil como una función de las variables de estrés aplicado.

Los modelos de aceleración más utilizados son: Modelo de Arrhenius, Modelo de Eyring y Ley de la Potencia Inversa.

\subsubsection{Modelo de Arrhenius}

El modelo de Arrhenius se utiliza en pruebas aceleradas de componentes en los que el mecanismo de fallo analizado se puede acelerar por temperatura. Resulta muy útil en ensayos acelerados de componentes electrónicos, porque muchos de los mecanismos de fallos de estos componentes se aceleran por temperatura.

El modelo de Arrhenuis se representa por la siguiente ecuación

$$
t_{p}=k e^{\frac{c}{t e m p}}
$$

Donde:

$t_{p}$ es el tiempo en el cual una proporción $p$ de la población falla.

temp es la temperatura absoluta (medida en grados Kelvin, ${ }^{\circ} K={ }^{o} C+273.16$ ). $k$ y $c$ son constantes.

En el siguiente ejemplo vamos a ver cómo determinar las constantes del modelo de Arrhenius, a partir del décimo cuantil de la distribución del tiempo de falla. La estimación de las constantes del modelo es independiente del $p$-cuantil que se considere; no obstante $t_{10}$ y $t_{50}$ son los que más se utilizan en la práctica.

Ejemplo 1.8.2. Un componente fue probado en cuatro diferentes temperaturas. Los parámetros estimados de la distribución Weibull fueron los siguientes: 


\begin{tabular}{|c|c|c|c|c|}
\hline Temperatura & $30^{\circ} \mathrm{C}$ & $50^{\circ} \mathrm{C}$ & $100^{\circ} \mathrm{C}$ & $150^{\circ} \mathrm{C}$ \\
\hline$\beta$ & 3.16 & 3.08 & 3.14 & 3.10 \\
$\lambda$ & 0.0011 & 0.0024 & 0.0051 & 0.0096 \\
\hline
\end{tabular}

Vamos a determinar la constantes del modelo de Arrhenius y estimaremos el factor de aceleración para una temperatura de $70^{\circ} \mathrm{C}$. Se supone que $20^{\circ} \mathrm{C}$ es la temperatura a la cual el componente opera en condiciones normales.

Solución:

Primero vamos a determinar el cuantil t $t_{10}$ para cada una de las distribuciones de Weibull. Para esta distribución se tiene que

$$
t_{p}=\frac{1}{\lambda}(-\ln (1-p))^{\frac{1}{\beta}}
$$

Entonces, los cuantiles obtenidos son

\begin{tabular}{|c|c|c|c|c|}
\hline Temperatura & $30^{\circ} \mathrm{C}$ & $50^{\circ} \mathrm{C}$ & $100^{\circ} \mathrm{C}$ & $150^{\circ} \mathrm{C}$ \\
\hline$t_{10}$ & 447.52 & 196.93 & 96.34 & 50.61 \\
\hline
\end{tabular}

Para poder determinar las constantes vamos a linealizar el modelo de Arrhenius, con lo que nos queda

$$
\ln t_{p}=\frac{c}{t e m p}+\ln k
$$

Podemos observar que esta última ecuación es de la forma $y=a x+b$, con $a=c y$ $b=\ln k$. Estimamos los valores de las dos constantes usando el método de regresión lineal simple, considerando $\frac{1}{\text { temp }}$ como variable independiente $y \ln t_{p}$ como variable dependiente. En la siguiente tabla se muestra la información requerida para usar el método de regresión lineal.

\begin{tabular}{|c|c|c|c|c|}
\hline$t_{10}$ & $\ln \left(t_{10}\right)$ & Temperatura $^{\circ} \mathrm{C}$ & Temperatura $^{\circ} \mathrm{K}$ & $\frac{1}{\text { temp }}$ \\
\hline 447.52 & 6.1037 & 30 & 303.16 & 0.0032986 \\
196.93 & 5.2828 & 50 & 323.16 & 0.0030944 \\
96.34 & 4.5679 & 100 & 373.16 & 0.0026798 \\
50.61 & 3.9241 & 150 & 423.16 & 0.00023632 \\
\hline
\end{tabular}

Los parámetros de la línea recta que mejor se ajustan a los datos son a $=2207.8$ y $b=-1.3425$. Entonces las constantes del modelo de Arrhenius son $k=e^{b}=e^{-1.3425}=0.2615$ y $c=2207.8$ 
Para terminar, calculamos el factor de aceleración para una temperatura de $70^{\circ} \mathrm{C}$. Sabemos que $t_{o}=\epsilon t_{\epsilon}$. Se usan los cuantiles $t_{10}$ en $20^{\circ} \mathrm{C}$ y $70^{\circ} \mathrm{C}$ y se tiene

$$
\epsilon=\frac{t_{10,20}}{t_{10,70}}
$$

El estimador para $t_{10}$ en $20^{\circ} \mathrm{C}$ es

$$
t_{10,20}=0.2612 e^{\frac{2207.8}{20+273.16}}=487.15
$$

El estimador para $t_{10}$ en $70^{\circ} \mathrm{C}$ es

$$
t_{10,70}=0.2612 e^{\frac{2207.8}{70+273.16}}=162.20
$$

Finalmente el factor de aceleración en $70^{\circ} \mathrm{C}$ es

$$
\epsilon=\frac{487.15}{162.20}=2.996
$$

\subsubsection{Modelo de Eyring}

Este modelo supone que existen varios factores que provocan estrés en el componente y aceleran el fallo. Uno de estos factores es siempre la temperatura, mientras que los otros pueden ser el campo eléctrico, el voltaje, la humedad, el estrés mecánico, la corriente eléctrica, ciclos de temperatura, etc. Dos factores comúnmente usados son la temperatura y la humedad.

La forma simple del modelo de Eyring, donde sólo se consideran dos factores, temperatura y voltaje, se caracteriza por la siguiente ecuación

$$
t_{p}=a e^{\frac{b}{t e m p}} V^{-c}
$$

Donde:

$t_{p}$ es el tiempo en el cual una proporción $p$ de la población falla.

temp es la temperatura absoluta (medida en grados Kelvin, ${ }^{\circ} K={ }^{o} C+273.16$ ).

$V$ es el voltaje.

$a, b$ y $c$ son constantes.

Para poder encontrar los valores de las tres constantes a, b y c se linealiza la ecuación del modelo (Ec. 1.17). Se obtiene la siguiente ecuación

$$
\ln t=\ln a+\frac{b}{t e m p}+c(-\ln V)
$$


Observe que esta última ecuación es de la forma $y=a+b x_{1}+c x_{2}$. Como ahora se tienen dos variables independientes utilizamos el método de regresión lineal múltiple para obtener los parámetros del plano que mejor se ajuste a un conjunto de datos, suponiendo $\ln t$ como la variable dependiente y $\frac{1}{t e m p} \mathrm{y}-\ln V$ como las variables independientes $x_{1}$ y $x_{2}$ respectivamente.

Ejemplo 1.8.3. Se realizan pruebas a un componente en tres niveles de temperatura y voltaje diferentes, los cuales se muestran en la siguiente tabla junto con los valores de $t_{50}$. Vamos a determinar los valores de las constantes del modelo de Eyring.

\begin{tabular}{|c|c|c|}
\hline$t_{50}$ & Temperatura $^{\circ} \mathrm{C}$ & Voltaje \\
\hline 45 & 30 & 20 \\
38 & 60 & 20 \\
35 & 30 & 40 \\
29 & 60 & 40 \\
23 & 45 & 30 \\
\hline
\end{tabular}

En la siguiente tabla se muestran los datos necesarios para realizar el análisis de regresión lineal múltiple.

\begin{tabular}{|c|c|c|c|c|c|c|}
\hline$t_{50}$ & $\ln (t)$ & $T\left(^{\circ} \mathrm{C}\right)$ & $T\left(^{\circ} \mathrm{K}\right)$ & $V$ & $1 / T\left(^{\circ} \mathrm{K}\right)$ & $-\ln V$ \\
\hline 45 & 3.8066 & 30 & 303.16 & 20 & 0.00329 & -2.9957 \\
38 & 3.6376 & 60 & 333.16 & 20 & 0.00300 & -2.9957 \\
35 & 3.5553 & 30 & 303.16 & 40 & 0.00329 & -3.6889 \\
29 & 3.3673 & 60 & 333.16 & 40 & 0.00300 & -3.6889 \\
23 & 3.4965 & 45 & 318.16 & 30 & 0.00314 & -3.4012 \\
\hline
\end{tabular}

Después de realizar el análisis de regresión lineal múltiple en el paquete Minitab se obtienen los siguientes valores para la ecuación del plano que mejor se ajusta a los datos: $a=2.95, b=601.52$ y $c=0.383$. Con estos valores se procede como en el ejemplo anterior para obtener el factor de aceleración $\epsilon$.

\subsubsection{Ley de la Potencia Inversa}

Este modelo resulta de utilidad cuando la vida del componente es inversamente proporcional al estrés aplicado. Se utiliza frecuentemente con estrés de tensión y estrés de fatiga por estrés cíclico, como por ejemplo vibración mecánica. La ecuación que caracteriza este modelo es 


$$
t_{p}=\frac{A}{S^{c}}
$$

Donde:

$t_{p}$ es el tiempo en el cual una proporción $p$ de la población falla.

$S$ es el estrés aplicado.

$A$ y $c$ son constantes.

Esta ecuación es una simplificación del modelo de Eyring, sólo que en éste no se considera la temperatura.

La forma linealizada del modelo que nos permitirá encontrar los valores de las constantes $A$ y $c$ es

$$
\ln t_{p}=\ln A-\ln S
$$




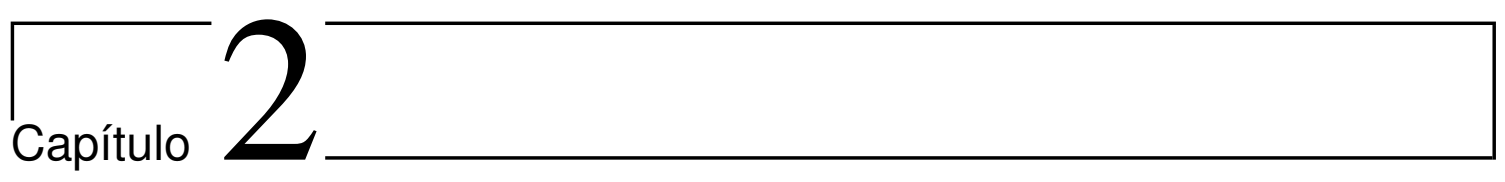

\section{Mantenimiento}

Hoy en día las empresas buscan asegurar y mejorar su competitividad por medio de esfuerzos, acciones y decisiones orientadas que garanticen equipos o sistemas que operen de manera eficiente y eficaz; clientes y usuarios satisfechos; riesgos reducidos; mínimos incidentes ambientales y costos óptimos.

El análisis RAMS incluye todas las consideraciones que deben tomarse en cuenta para poder desarrollar un nuevo producto o servicio. RAMS agrupa cuatro conceptos: Confiabilidad (Reliability), Disponibilidad (Availability), Mantenibilidad (Maintainability) y Seguridad (Safety). La confiabilidad se define como la capacidad de que un equipo o sistema opere sin fallar durante un periodo determinado y bajo ciertas condiciones previamente establecidas; en consecuencia, si estas condiciones cambian, la confiabilidad del sistema también cambiará. La Disponibilidad es la capacidad del sistema para funcionar en un determinado instante. La Mantenibilidad es la capacidad de ser mantenido o reparado preventiva y correctivamente con objeto de mejorar su disponibilidad. Durante el proceso de diseño se debe considerar la mantenibilidad como un criterio fundamental para conseguir calidad de servicio y ahorro de costes en el futuro. La experiencia ha demostrado que un ahorro en la inversión inicial puede suponer un gasto mucho mayor en mantenimiento. Por último se considera la Seguridad, que se define como la capacidad de operar sin producir daños al usuario o a su entono.

El usuario considera que un componente o sistema es confiable si realiza adecuadamente la tarea para la que ha sido diseñado a lo largo de su vida útil. En realidad no existen equipos totalmente confiables, pues siempre hay una probabilidad de falla, la cual debe reducirse hasta valores suficientemente pequeños los cuales permitan la aceptación de los dispositivos por parte de la usuarios.

Las fallas de un componente pueden clasificarse según diferentes criterios. Por 
la forma en que aparecen pueden ser catastróficas o por degradación. Las fallas catastróficas son consecuencia de un cambio brusco en un parámetro o característica operativa del componente, mientras que las fallas por degradación se deben al desgaste de los materiales. Según el momento en que aparecen pueden ser: fallas infantiles, fallas por azar o aleatorias y fallas por desgaste u obsolescencia. Las fallas infantiles se producen en productos recién salidos de fábrica, y son la consecuencia de los defectos de fabricación no detectados en la inspección. Este tipo de fallas pueden disminuirse con un buen control de calidad y son las que normalmente cubre la garantía. Las fallas por azar se deben a circunstancias desfavorables para el componente o sistema y se pueden presentar durante toda su vida útil. Las fallas por desgaste se presentan después de que el sistema ha tenido un largo periodo de uso. Si se considera la relación entre las fallas, pueden ser primarias o secundarias. Son primarias cuando se producen sin que ninguna otra falla las haya provocado, y secundarias cuando fueron originadas por una primera falla.

Para disminuir el impacto negativo de los procesos de desgaste de un componente, se aplican tareas de mantenimiento preventivo, las cuales tienen por objetivo anticiparse a la falla para evitar problemas y al mismo tiempo asegurar que el componente o sistema funcione de manera continua y en las mejores condiciones.

\subsection{Tipos de Mantenimiento}

El mantenimiento se define como la combinación de actividades mediante las cuales un equipo o sistema se mantiene, o se restablece a un estado en el que puede realizar las funciones para la cual fue diseñado o asignado. El objetivo del mantenimiento es mantener la confiabilidad del sistema, es decir, conservarlo de la mejor manera posible.

Hay dos categorías básicas de mantenimiento: mantenimiento correctivo y mantenimiento preventivo o planificado.

\subsection{Mantenimiento Correctivo}

El mantenimiento correctivo se realiza después que ocurre una falla, es decir, cuando el equipo es incapaz de seguir operando o no está cumpliendo la función para 
cual fue diseñado o asignado. El objetivo de este tipo de mantenimiento consiste en llevar los equipos después de una falla, a sus condiciones operativas, por medio de restauración o reemplazo de componentes o partes [6]. En este tipo de mantenimiento se incluyen las tareas no programadas, que son consecuencia de una falla repentina en el sistema o producto, como son:

- Identificación de la falla

- Localización del elemento en falla

- Desensamble

- Reemplazo o arreglo

- Ensamble

- Pruebas

- Verificación

\subsection{Mantenimiento Preventivo}

El mantenimiento preventivo se define como una serie de tareas planeadas y programadas previamente con base en el tiempo, el uso o la condición del equipo, y que se realizan a fin de evitar la ocurrencia de las fallas. Su objetivo es mantener los equipos bajo condiciones específicas de operación [6]. Las tareas que se generan con este tipo de mantenimiento incluyen las siguientes actividades:

- Desensamble

- Reemplazo o arreglo

- Ensamble

- Pruebas

- Verificación

Se ha mencionado antes que el comportamiento de la tasa de falla de un componente se puede dividir en tres etapas: infantil, de vida útil y de desgaste. ¿Qué beneficios se obtienen si se realiza el mantenimiento preventivo en cada una de estas etapas? Para la etapa infantil en donde la ocurrencia de fallas se debe principalmente a defectos de fabricación, las actividades de mantenimiento preventivo incrementarían la 
tasa de falla, puesto que el mantenimiento restaura el componente o sistema al estado inicial, entonces el sistema se restaura a un estado en el cual se tiene una tasa de falla alta y por ende, aumentaría el número de fallas. Respecto a la etapa de vida útil, el mantenimiento preventivo únicamente originaría pérdida de tiempo y dinero, pues en esta etapa las fallas son consecuencia de averías accidentales debidas a factores externos. Las fallas que si se pueden prevenir con mantenimiento son las de la etapa de desgaste, ya que en esta etapa la tasa de fallas es creciente, es decir, depende de la edad del componente; por lo tanto el mantenimiento preventivo restaura el sistema a un estado en el cual la tasa de fallas es mas baja.

De acuerdo a lo dicho en el párrafo anterior, se establece una diferencia entre los equipos electrónicos, cuyos componentes básicamente responden a fallas por causas completamente aleatorias, y los equipos mecánicos, en los cuales domina la falla por desgaste. En los equipos electrónicos el mantenimiento preventivo normalmente no sólo no aporta ventaja alguna, sino que llevaría a desperdiciar gran parte de la vida útil de los componentes, agregando el costo de la acción preventiva.

\subsection{Función de densidad de fallo que resulta cuando se practican acciones de mante- nimiento preventivo}

Sea $f(t)$ una función de densidad de fallas que da origen a una tasa de fallas creciente con el tiempo. Se quiere hallar la función de densidad de fallas $f_{M P}(t)$ que resulta cuando se practican acciones de mantenimiento preventivo.

Las acciones de mantenimiento preventivo, que se realizan a intervalos de tiempo $t_{p}$, determinan una nueva función de densidad de fallas, pues los componentes sólo pueden fallar dentro del intervalo $\left[0, t_{p}\right]$ después del cual se lleva a cabo el mantenimiento preventivo.

Sea

$$
f_{1}(t)= \begin{cases}f(t), & \text { si } 0 \leq t \leq t_{p} \\ 0, & \text { si } t>t_{p}\end{cases}
$$

Vamos a describir la nueva función de densidad de fallos, $f_{k}(t)$, para cualquier instante $t>t_{p}$. 
Observe que la función $f_{1}(t)$ nos permite calcular la probabilidad de fallo en el intervalo $\left[0, t_{p}\right]$. De esta forma, la función de confiabilidad obtenida con base en $f(t)$ sólo tiene validez en el intervalo $\left[0, t_{p}\right]$; denotemos ésta como $R_{0}(t)$.

Debido a que después de una intervención (correctiva o preventiva) el equipo queda como nuevo, si consideramos entonces la intervención $k$ tendremos

$$
\lim _{\Delta t \rightarrow 0} R\left(k t_{p}+\Delta t\right)=1, \quad k=0,1,2, \cdots, n
$$

Por tanto, dentro de cualquier intervalo $\left(k t_{p},(k+1) t_{p}\right)$, a partir de una intervención, la función de densidad de fallos será una réplica de la densidad de fallos entre 0 y $t_{p}$.

Sabemos que $R\left(t_{p}\right)$ es la probabilidad de que el componente no falle durante un intervalo cualquiera $\left[0, t_{p}\right]$. Entonces, ¿cuál será la probabilidad de que el componente siga en funcionamiento en un instante $t$ cualquiera posterior a $k t_{p}$ ?

$$
t=k t_{p}+t_{m}, \quad k=1,2,3, \ldots, n, \quad 0<t_{m}<t_{p}, \quad t_{m}=t-k t_{p}
$$

Ésta será la probabilidad de que el sistema haya funcionado desde el instante $t=0$ hasta el instante $t=k t_{p} \mathrm{y}$ de que no se produzca un fallo en el intervalo

$$
k t_{p}<t<k t_{p}+t_{m}
$$

Como la confiabilidad en el intervalo $\left(0, t_{p}\right)$ es $R\left(t_{p}\right)$, la probabilidad de que no haya fallado en ninguno de los $k$ intervalos anteriores es

$$
R\left(k t_{p}\right)=\left[R\left(t_{p}\right)\right]^{k}
$$

Por otro lado,

$$
R\left(t_{m}\right)=1-F\left(t_{m}\right)=1-\int_{0}^{t_{m}} f_{1}(t) d t
$$

Entonces la función de confiabilidad en $t$ con mantenimiento preventivo $R_{M P}$ es

$$
\begin{aligned}
R_{M P}(t) & =R\left(k t_{p}+t_{m}\right) \\
& =R\left(k t_{p}\right) R\left(t_{m}\right) \\
& =\left[R_{o}\left(t_{p}\right)\right]^{k} R_{o}\left(t-k t_{p}\right)
\end{aligned}
$$

Por lo tanto, la función de densidad de fallo $f_{k}(t)$ dentro del intervalo 
$\left(k t_{p},(k+1) t_{p}\right)$ estará dada por

$$
\begin{aligned}
f_{k}(t) & =-\frac{d R_{M P}(t)}{d t} \\
& =-\frac{d\left[R_{o}\left(t_{p}\right)\right]^{k} R_{o}\left(t-k t_{p}\right)}{d t} \\
& =-\left[R_{o}\left(t_{p}\right)\right]^{k} \frac{d R_{o}\left(t-k t_{p}\right)}{d t} \\
& =\left[R_{o}\left(t_{p}\right)\right]^{k} f_{1}\left(t-k t_{p}\right)
\end{aligned}
$$

Finalmente, la función de densidad del tiempo de falla cuando se efectúa mantenimiento preventivo, se puede escribir como:

$$
f_{M P}(t)=\sum_{k=0}^{\infty} f_{k}(t)=\sum_{k=0}^{\infty}\left[R_{o}\left(t_{p}\right)\right]^{k} f_{1}\left(t-k t_{p}\right), \quad t \geq 0
$$

Es decir, la probabilidad instantánea de que ocurra una falla en un instante $t=k t_{p}+t_{m}$ con $0<t_{m}<t_{p}$ y $k=1,2,3, \ldots$, siendo que en los instantes $t=k t_{p}$ se realiza mantenimiento preventivo, está dada por la suma de las densidades de fallo en cada uno de los intervalos $\left(k t_{p},(k+1) t_{p}\right)$.

Se puede observar que $f_{M P}(t) \geq 0 \quad \forall t \in[0,+\infty)$ y además

$$
\begin{aligned}
\int_{0}^{\infty} f_{M P}(t) d t & = \\
& =\int_{0}^{\infty} \sum_{k=0}^{\infty}\left[R\left(t_{p}\right)\right]^{k} f_{1}\left(t-k t_{p}\right) d t \\
& =\sum_{k=0}^{\infty}\left[R\left(t_{p}\right)\right]^{k} \int_{k}^{(k+1)} f_{1}\left(t-k t_{p}\right) d t \\
& =\frac{1}{1-R\left(t_{p}\right)} \int_{0}^{\left(t_{p}\right)} f(t) d t \\
& =\frac{1}{F\left(t_{p}\right)} F\left(t_{p}\right) \\
& =1
\end{aligned}
$$

Hay que notar que el efecto del mantenimiento preventivo periódico es alterar la función de densidad de falla de su forma original a una de tipo exponencial. Por tanto, la tasa de falla que en un principio era creciente, con el tiempo se puede aproximar a una tasa de falla constante, que no crece. Esto es, el mantenimiento preventivo periódico elimina las fallas por desgaste u obsolescencia del dispositivo, dejando la posibilidad de que sólo ocurran fallas que puedan ser originadas por algún fenómeno 
imprevisto.

Ejemplo 2.4.1. Un componente tiene una función de densidad de falla $f(t)$ que tiene una distribución uniforme entre [0,10] años, es decir,

$$
f(t)= \begin{cases}\frac{1}{10}, & \text { si } 0 \leq t \leq 10 \\ 0, & \text { si } t>10\end{cases}
$$

El componente es sometido a mantenimiento preventivo 1 vez al año. Vamos a calcular la función de densidad resultante cuando se considera el efecto del mantenimiento, para después comparar el MTBF original y el MTBF $F_{M P}$ que resulta cuando se efectúa el mantenimiento preventivo.

A partir de la función de densidad de fallo se tiene que la función de distribución de fallo es

$$
F(t)= \begin{cases}\frac{t}{10}, & \text { si } 0 \leq t \leq 10 \\ 1, & \text { si } t>10\end{cases}
$$
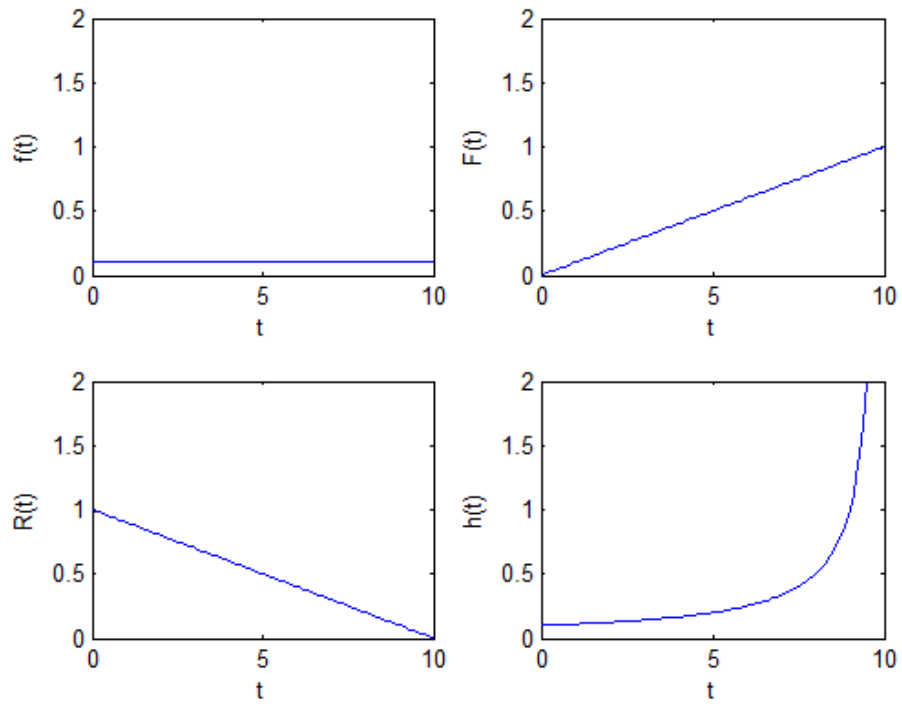

Figura 2.1: Funciones estadísticas sin efecto del mantenimiento

La función de confiabilidad es

$$
R(t)=1-\frac{t}{10}, \quad 0 \leq t \leq 10
$$


Y la tasa de fallo queda como sigue:

$$
h(t)=\frac{0.1}{1-0.1 t}=\frac{1}{10-t}
$$

En la Figura 2.1 se muestran las funciones de densidad, distribución, confiabilidad y tasa de falla del componente, sin considerar el efecto del mantenimiento.

Para calcular el MTBF hacemos

$$
\begin{aligned}
\int_{0}^{10} R(t) d t & =\int_{0}^{10}(1-0.1 t) d t \\
& =\int_{0}^{10} d t-0.1 \int_{0}^{10} t d t \\
& =10-\left.0.1\left(\frac{t^{2}}{2}\right)\right|_{0} ^{10} \\
& =10-0.1\left(\frac{10^{2}}{2}\right) \\
& =5
\end{aligned}
$$

Es decir, el tiempo medio entre dos fallos consecutivos es de 5 años.

Considerando que el mantenimiento preventivo se efectúa cada año, $t_{p}=1$ y entonces $R(1)=0.9$.

De ahí, que la función de densidad de fallo que estamos buscando es

$$
f_{M P}(t)=\sum_{k=0}^{\infty}(0.9)^{k}(0.1), \quad k t_{p}<t<(k+1) t_{p}
$$

Nótese que debido al mantenimiento preventivo, el componente recupera su distribución uniforme en $[0,10]$ cada año.

Ahora vamos a calcular el MTBF $F_{P P}$ como $\frac{1}{h^{*}(t)}$. La nueva tasa de falla, $h^{*}(t)$ se puede aproximar con el valor medio de $h(t)$ en el intervalo [0,1]. Es decir

$$
h^{*}(t)=\int_{0}^{1} \frac{d t}{10-t}
$$

Si hacemos el cambio de variable

$$
u=10-t \quad d u=-d t
$$




$$
\begin{aligned}
h^{*}(t) & =\int_{9}^{10} \frac{d u}{u} \\
& =\ln 10-1 n 9=0.1053
\end{aligned}
$$

Por lo tanto, la aproximación que se tiene para el valor del MTBF $F_{M P}$ que se obtiene cuando se considera el efecto del mantenimiento preventivo es

$$
\frac{1}{0.1053}=9.491
$$

El valor exacto de $M T B F_{M P}$ es la media de la variable $t$ con densidad $f_{M P}(t)$, es decir,

$$
\begin{aligned}
\int_{0}^{\infty} t f_{M P}(t) d t & = \\
& =\int_{0}^{\infty} t \sum_{k=0}^{\infty}(0.1)(0.9)^{k} d t \\
& =\frac{1}{10} \sum_{k=0}^{\infty}(0.9)^{k} \int_{k}^{k+1} t d t \\
& =\frac{1}{20} \sum_{k=0}^{\infty}(0.9)^{k}(2 k+1) \\
& =9.5
\end{aligned}
$$

Este valor 9.5 años representa un gran incremento de tiempo, respecto de $M T B F=5$ años que se obtienen cuando no se tiene en cuenta el mantenimiento preventivo.

\subsection{Función de Mantenibilidad}

El tiempo empleado para la reparación del dispositivo averiado depende de las características del sistema en falla y de factores tales como la accesibilidad, facilidades para el diagnóstico, habilidad de los reparadores, etc. Como las fallas son aleatorias, y es diferente el tiempo de reparación involucrado en cada dispositivo, los tiempos necesarios para reparar el dispositivo también serán aleatorios. 
Considérese la variable aleatoria $T T R$ que representa el tiempo de ejecución de las tareas de mantenimiento, ya sea preventivo o correctivo. Esta variable toma valores en el intervalo $[0,+\infty)$.

La función de distribución de la variable aleatoria $T T R$ se denomina función de mantenibilidad y se denota por $M(t)$, es decir

$$
M(t)=P(T T R<t)=\int_{0}^{t} m(x) d x
$$

donde $m(x)$ es la función de densidad de la variable aleatoria TTR. La función de mantenibilidad indica la probabilidad de que un sistema que entró en proceso de reparación en el instante $t=0$ este reparado al cabo de un cierto tiempo $t$. La experiencia indica que la distribución lognormal representa a $M(t)$; la asimetría de la función indica que tan rápido se llevan a cabo las actividades de mantenimiento.

Ejemplo 2.5.1. El tiempo de reparación de un sistema sigue una distribución lognormal con parámetro de escala $\sigma=1.2$ y parámetro de localización $\mu=2.2$ horas. El parámetro $\mu$ es la media del logaritmo natural de los tiempos de reparación y $\sigma$ es la desviación estándar del logaritmo natural de los tiempos de reparación alrededor de $\mu$.

La probabilidad de completar la reparación del sistema en un tiempo menor a 5 horas está dada por $M(5)$ como se muestra a continuación.

\section{Entonces}

Sabemos que $m(x)=\frac{1}{\sigma \sqrt{2 \pi} x} e^{-\frac{1}{2}\left(\frac{\ln x-\mu}{\sigma}\right)^{2}} \quad x>0$

$$
\begin{aligned}
M(5) & =\int_{0}^{5} \frac{1}{1.2 \sqrt{2 \pi} x} e^{-\frac{1}{2}\left(\frac{\ln x-2.2}{1.2}\right)^{2}} d x \\
& =\Phi\left(\frac{\ln 5-2.2}{1.2}\right) \\
& =\Phi(-0.4921) \\
& =0.3113
\end{aligned}
$$

Por lo tanto, la probabilidad de efectuar las tareas de mantenimiento en menos de 5 horas es 0.3113 . 


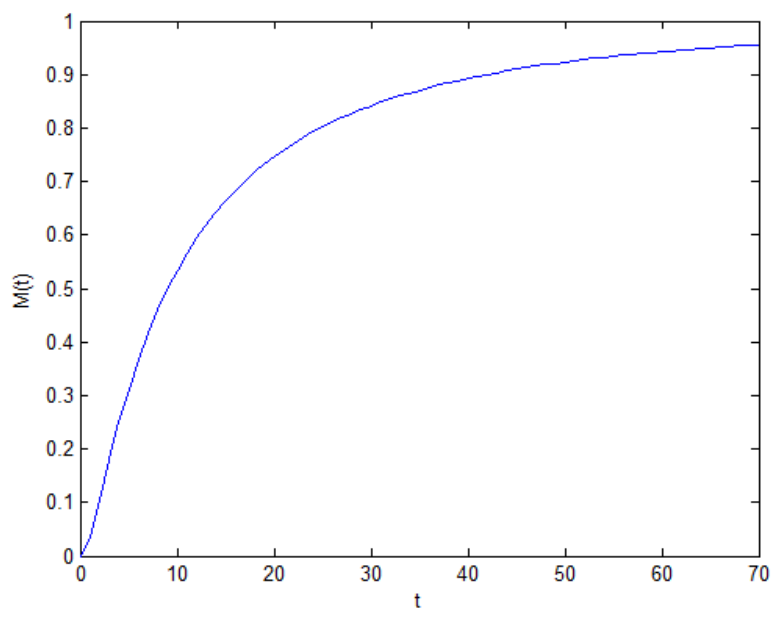

Figura 2.2: Función de Mantenibilidad

En la Figura 2.2 se muestra el comportamiento de la función de mantenibilidad para este sistema.

Al igual que en el área de confiabilidad, se denominará tasa de mantenibilidad $\gamma(t)$ a la probabilidad condicional de completar las acciones de mantenimiento durante el intervalo de tiempo $(t+\Delta t)$, con $\Delta t \rightarrow 0$, suponiendo que la acción comenzada en el instante $t=0$ no haya sido completada antes del tiempo $t$. Ésta se representa por

$$
\gamma(t)=\frac{m(t)}{1-M(t)}
$$

Otra de las medidas importantes es el tiempo medio de la duración de las acciones de mantenimiento MTTR. Analíticamente se expresa por

$$
M T T R=E(T T R)=\int_{0}^{\infty} t m(t) d t
$$

Ejemplo 2.5.2. Consideremos que la tasa de mantenibilidad $\gamma(t)$ de un sistema es constante. Entonces se tienen las siguientes ecuaciones:

$$
\begin{gathered}
M(t)=1-e^{-\gamma t} \\
m(t)=\gamma e^{-\gamma t}
\end{gathered}
$$




$$
\operatorname{MTTR}=\frac{1}{\gamma}
$$

Para esta distribución, la mejor estimación de mantenibilidad del sistema se puede obtener mediante el cociente entre la suma de los tiempos de reparación $t_{r}$ y el número total de fallos $n$, es decir,

$$
\operatorname{MTTR}=\frac{\sum_{r=0}^{n} t_{r}}{n}
$$

Ejemplo 2.5.3. Se sabe que durante un periodo de un mes hubo 15 acciones de mantenimiento no programadas y que se requirieron 1200 minutos para efectuar las reparaciones. Por datos históricos, el analista de mantenimiento sabe que los tiempos de mantenimiento se distribuyen de manera exponencial. ¿Cuál es el valor medio de los tiempos de reparación?

Se tiene que la suma de los tiempos de reparación es 1200 y como fueron 15 intervenciones no programadas, entonces $n=15$. Por lo tanto

$$
\operatorname{MTTR}=\frac{1200}{15}=80
$$

Es decir, las actividades de mantenimiento no programado requirieron en promedio 80 minutos.

Ejemplo 2.5.4. Consideremos ahora que $m(t)$, la función de densidad de los tiempos de reparación es lognormal. Se tienen las siguientes ecuaciones:

$$
\begin{gathered}
M(t)=\int_{0}^{t} \frac{1}{\sigma \sqrt{2 \pi} x} e^{-\frac{1}{2}\left(\frac{\ln x-\mu}{\sigma}\right)^{2}} d x \\
m(t)=\frac{1}{\sigma \sqrt{2 \pi} t} e^{-\frac{1}{2}\left(\frac{\ln t-\mu}{\sigma}\right)^{2}}
\end{gathered}
$$

Recordemos que

$$
\gamma(t)=\frac{m(t)}{1-M(t)}
$$




$$
\operatorname{MTTR}=e^{-\left(\mu+\frac{\sigma^{2}}{2}\right)}
$$

En esta distribución

$$
\hat{\mu}=\frac{\sum_{r=0}^{n} \ln t_{r}}{n}
$$

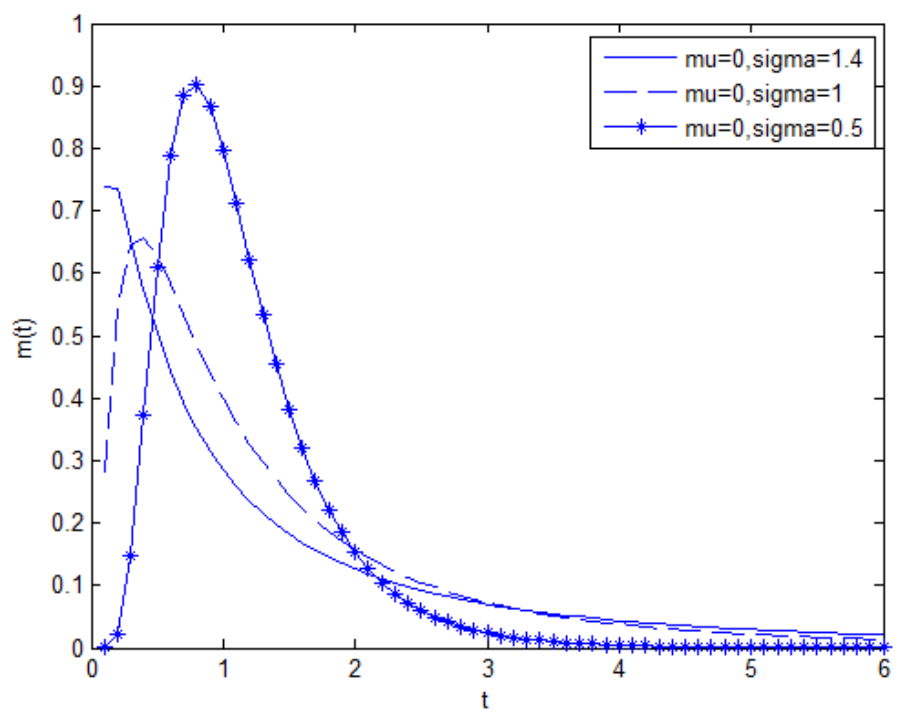

Figura 2.3: Función de densidad lognormal

En la Figura 2.3 se muestran las gráficas de la función de densidad $m(t)$ con diferentes parámetros. Se observa que la probabilidad se concentra cerca de cero y que después de la moda, la probabilidad decrece. También se puede observar que a medida que decrece el valor del parámetro $\sigma$ la distribución se vuelve más simétrica. 


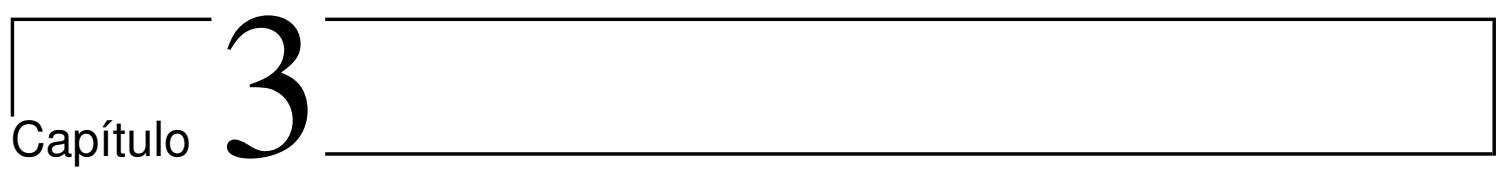

\section{Modelos Matemáticos de Políticas Óptimas de Mantenimiento con tiempos de reparación instantáneos}

Como el fenómeno de aparición de fallas se comporta como un proceso aleatorio, está claro que no podemos llegar a predecir cuando ocurrirán las fallas, pero sí podemos determinar con base en la mejor información los tiempos de mantenimiento preventivo y las políticas de mantenimiento más adecuadas a largo plazo.

Los propietarios de un sistema deben decidir acerca de sus políticas de mantenimiento o realizarlas utilizando datos proporcionados por su sistema, no basados en intuiciones, pues esto puede ocasionar pérdida de confiabilidad del sistema.

En las siguientes secciones se estudian dos de las políticas de mantenimiento preventivo introducidas por Richard E. Barlow y Proschan (1965) [7], las cuales tienen por objetivo lograr un balance entre la disminución del riesgo de llegar a tener una falla y el aumento de los costos de mantenimiento preventivo; de ahí que estos modelos se construyen para determinar el intervalo óptimo entre dos reemplazos sucesivos de componentes. Usaremos reemplazo para referirnos a mantenimiento preventivo o mantenimiento por falla. 


\subsection{Política I. Mantenimiento Basado en la Edad}

La falla de un sistema durante su operación puede ser de poca o mucha importancia dependiendo del tipo de falla, llegando a ser muy costoso y peligroso.Una de las áreas importantes de la teoría de confiabilidad es el estudio de las políticas de mantenimiento para reducir los costos de operación.

En esta política de mantenimiento por edad, ya sea que el sistema es reemplazado en el tiempo de falla $t$, si $t<t_{p}$, o una vez que el sistema ha alcanzado una edad de operación $t_{p}$, lo que ocurra primero. Se supone que en ambos casos, luego del reemplazo, el sistema queda en el estado "as good as new". Es decir, cada reemplazo, que puede ser mantenimiento preventivo o mantenimiento por falla constituye una renovación del sistema.

La edad a la cual el sistema es reemplazado depende, entre otros, de los siguientes factores:

- Distribución del tiempo de falla.

- Costos de reemplazo por falla.

- Costos de reemplazo preventivo.

- Tiempo de inactividad por falla y reemplazo preventivo.

- Medida de efectividad: minimizar costos, maximizar disponibilidad, o lograr cierta confiabilidad.

Para este modelo vamos a suponer que los reemplazos preventivos son menos costosos que reemplazos por falla, pues además del costo de mantenimiento, se tiene el costo de reparar los daños ocasionados al sistema. También se considera que el costo del tiempo de inactividad asociado con las acciones de mantenimiento preventivo y reparaciones mínimas, las cuales se efectúan cuando el sistema falla, es insignificante, es decir, los reemplazos son instantáneos.

Es importante señalar que el modelo sólo será de utilidad en la etapa de desgaste u obsolescencia del componente o sistema, pues en esta etapa el sistema presenta una tasa de falla creciente.

Diremos que un ciclo se completa cada vez que se realiza un reemplazo ya sea por mantenimiento preventivo o por falla. Entonces para este modelo existen dos ciclos 
posibles: en el primer ciclo se realiza mantenimiento a la edad especificada $t_{p} \mathrm{y}$ en el segundo ciclo el sistema falla antes del mantenimiento preventivo.

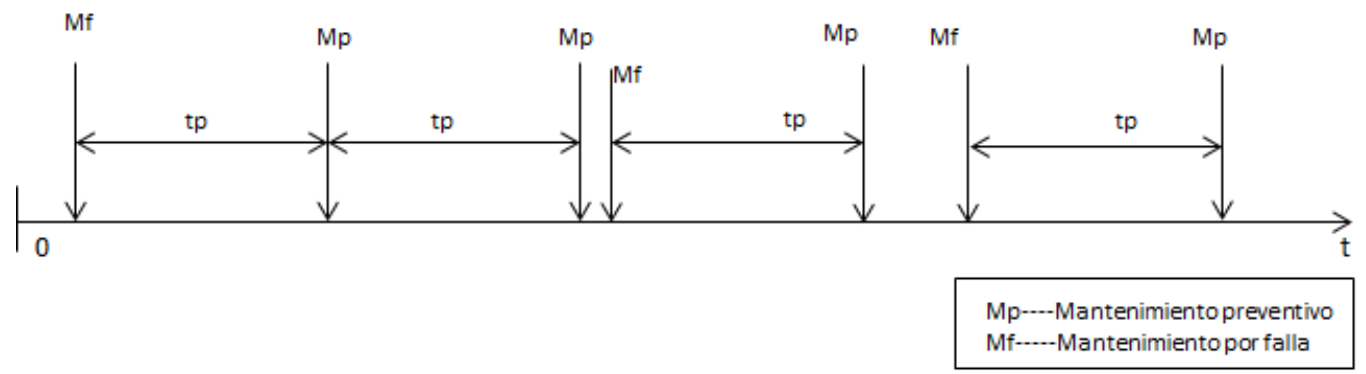

Figura 3.1: Reemplazo por edad

El objetivo de esta política es encontrar el valor de $t_{p}$ que minimize el costo total esperado por unidad de tiempo, el cual queda definido por la siguiente ecuación:

$$
C\left(t_{p}\right)=\frac{E(C)}{E(D)}
$$

Donde:

$C$ es el costo incurrido durante un ciclo.

$D$ es el largo de un ciclo.

$E(C)$ es el costo esperado de un ciclo.

$E(D)$ es el largo esperado de un ciclo.

Vamos a considerar

$$
\begin{gathered}
C= \begin{cases}C_{p}, & \text { si } T>t_{p} \\
C_{f}, & \text { si } T \leq t_{p}\end{cases} \\
D= \begin{cases}d_{p}+t_{p}, & \text { si } T>t_{p} \\
d_{f}+T, & \text { si } T \leq t_{p}\end{cases}
\end{gathered}
$$

Donde:

$C_{p}$ es el costo de mantenimiento preventivo.

$C_{f}$ es el costo de mantenimiento por falla.

$d_{p}$ es la duración del mantenimiento preventivo.

$d_{f}$ es la duración del mantenimiento por falla.

$T$ es el tiempo de falla.

$t_{p}$ es el tiempo de operación del componente sin falla después del cual se efectúa un 
mantenimiento preventivo.

Si suponemos que los tiempos requeridos para llevar a cabo tanto las actividades de mantenimiento preventivo como correctivo son nulos, es decir, que $d_{f}=d_{p}=0$ entonces

$$
D= \begin{cases}t_{p}, & \text { si } T>t_{p} \\ T, & \text { si } T \leq t_{p}\end{cases}
$$

Recordemos además que $C_{p}<C_{f}$.

Por lo dicho anteriormente, el costo total esperado por ciclo está dado por las dos probabilidades, que $T>t_{p}$, lo cual significa que no hay falla en el intervalo de mantenimiento preventivo, y que $T \leq t_{p}$, es decir, que ocurre una falla antes de efectuar el mantenimiento preventivo.

Entonces el costo total esperado por ciclo se expresa como sigue:

$$
C_{p} P\left(T>t_{p}\right)+C_{f} P\left(T \leq t_{p}\right)=C_{p} R\left(t_{p}\right)+C_{f}\left[1-R\left(t_{p}\right)\right]
$$

De la misma forma, el largo esperado de un ciclo está dado por las dos probabilidades, que $T>t_{p}$ y que $T \leq t_{p}$.

En el caso de que se realice una mantenimiento preventivo en $t_{p}$, el largo esperado del ciclo es $t_{p}$. En cambio, si se realiza mantenimiento preventivo antes de $t_{p}$, el largo esperado del ciclo es el tiempo medio entre falla, pero en el intervalo $\left[0, t_{p}\right]$. Se busca entonces la media de la distribución truncada en $t_{p}$.

Sabemos que para la distribución completa, el $M T B F=\int_{0}^{\infty} t f(t) d t$, pero como la función de densidad se trunca en $t_{p}$, el valor medio queda expresado de la siguiente forma

$$
M\left(t_{p}\right)=\int_{0}^{t_{p}} t \frac{f(t)}{1-R\left(t_{p}\right)} d t
$$

Por lo tanto, el largo esperada del ciclo, $E(D)$ está dado por

$$
t_{p} P\left(T>t_{p}\right)+M\left(t_{p}\right) P\left(T \leq t_{p}\right)=t_{p} R\left(t_{p}\right)+M\left(t_{p}\right)\left[1-R\left(t_{p}\right)\right]
$$

Hasta aquí, el costo total esperado por unidad de tiempo queda expresado como

$$
\begin{aligned}
C\left(t_{p}\right) & =\frac{C_{p} R\left(t_{p}\right)+C_{f}\left[1-R\left(t_{p}\right)\right]}{t_{p} R\left(t_{p}\right)+M\left(t_{p}\right)\left[1-R\left(t_{p}\right)\right]} \\
& =\frac{C_{p} R\left(t_{p}\right)+C_{f}\left[1-R\left(t_{p}\right)\right]}{t_{p} R\left(t_{p}\right)+\int_{0}^{t_{p}} t f(t) d t}
\end{aligned}
$$


La expresión anterior se puede simplificar aún más si realizamos integración por partes en

$$
\begin{gathered}
\quad \int_{0}^{t_{p}} t f(t) d t . \\
\begin{aligned}
u=t \quad d v=f(t) d t \\
d u=d t \quad v=F(t)=1-R(t)
\end{aligned} \\
\int_{0}^{t_{p}} t f(t) d t=\left.t\left[1-R\left(t_{p}\right)\right]\right|_{0} ^{t_{p}}-\int_{0}^{t_{p}}[1-R(t)] d t \\
=t_{p}-t_{p} R\left(t_{p}\right)-t_{p}+\int_{0}^{t_{p}} R(t) d t
\end{gathered}
$$

Entonces el largo esperado por ciclo queda definido por

$$
\int_{0}^{t_{p}} R(t) d t
$$

Finalmente, el costo esperado por unidad de tiempo para un intervalo de reemplazo $t_{p}$ es definido por la siguiente función de costo.

$$
C\left(t_{p}\right)=\frac{C_{p} R\left(t_{p}\right)+C_{f}\left[1-R\left(t_{p}\right)\right]}{\int_{0}^{t_{p}} R(t) d t}, t_{p} \in[0,+\infty)
$$

Es decir, el costo esperado por unidad de tiempo para un intervalo de reemplazo $t_{p}$ va a estar dado por la razón entre la suma del costo de mantenimiento preventivo multiplicado por la probabilidad de que no ocurra una falla es este intervalo más la probabilidad de que ocurra la falla multiplicada por el costo de mantenimiento por falla, y la confiabilidad acumulada hasta el instante $t_{p}$.

Como ya sabemos, el valor de $t_{p}$ que minimiza la ecuación anterior es aquel que satisface $\frac{d C\left(t_{p}\right)}{d t_{p}}=0$

Tenemos que

$$
\frac{d C\left(t_{p}\right)}{d t_{p}}=\frac{\left(\int_{0}^{t_{p}} R(t) d t\right)\left[C_{p} R^{\prime}\left(t_{p}\right)-C_{f} R^{\prime}\left(t_{p}\right)\right]-\left[C_{p} R\left(t_{p}\right)+C_{f}\left(1-R\left(t_{p}\right)\right)\right] R\left(t_{p}\right)}{\left(\int_{0}^{t_{p}} R(t) d t\right)^{2}}
$$

Si igualamos las derivada a cero nos queda que

$$
\left(\int_{0}^{t_{p}} R(t) d t\right)\left(-C_{p} f\left(t_{p}\right)+C_{f} f\left(t_{p}\right)\right)=C_{f} R\left(t_{p}\right)
$$




$$
\begin{aligned}
& \Rightarrow \\
& C_{f} f\left(t_{p}\right) \int_{0}^{t_{p}} R(t) d t-C_{p} f\left(t_{p}\right) \int_{0}^{t_{p}} R(t) d t=C_{p}\left[1-F\left(t_{p}\right)\right] \\
& \Rightarrow \\
& C_{f} h\left(t_{p}\right) \int_{0}^{t_{p}} R(t) d t-C_{p} h\left(t_{p}\right) \int_{0}^{t_{p}} R(t) d t=C_{p} \\
& \Rightarrow \\
& h\left(t_{p}\right) \int_{0}^{t_{p}} R(t) d t=\frac{C_{p}}{C_{f}-C_{p}}
\end{aligned}
$$

De esta última ecuación se puede observar que el valor de $t_{p}$ para el cual el costo total esperado por unidad de tiempo es mínimo, depende de la relación que existe entre los costos de mantenimiento preventivo y mantenimiento por falla.

Ejemplo 3.1.1. Considere un componente cuyos tiempos de falla son modelados con la distribución Weibull con parámetros $\lambda=0.0025$ y $\beta=2.93$. Vamos a analizar el comportamiento del intervalo óptimo de mantenimiento preventivo, a partir de la relación que existe entre los costos de mantenimiento preventivo y por falla.

Enseguida vamos a describir la ecuación que describe el costo total esperado por unidad de tiempo para un intervalo de reemplazo $t_{p}$.

Sabemos que $R(t)=e^{-(\lambda t)^{\beta}}$ es la función de confiabilidad para cualquier instante $t \geq 0$.

Entonces por (3.5)

$$
C\left(t_{p}\right)=\frac{C_{p} e^{-\left(\lambda t_{p}\right)^{\beta}}+C_{f}\left(1-e^{-\left(\lambda t_{p}\right)^{\beta}}\right)}{\int_{0}^{t_{p}} e^{-(\lambda t)^{\beta}} d t}
$$

En la Figura 3.2 se muestran las gráficas de la función del costo esperado por unidad de tiempo considerando $C_{p}=1$ y $C_{f}=k C_{p}$. Los valores de $k$ se asignan de tal forma que los costos de mantenimiento por falla aumenten, pero además que $C_{p}<k C_{f}$. En las gráficas podemos observar que la función del costo presenta un mínimo una vez que $k$ toma valores por arriba de 1.4 . De no ser asi entonces $t_{p}=\infty$, lo cual indica que conviene el reemplazo sólo en la falla.

En la Figura 3.3 se puede observar la relación entre el valor de la constante de proporcionalidad $k$ y el $t_{p}$ óptimo, que indica la frecuencia con la que se debe efectuar el mantenimiento preventivo. Observamos que a medida que aumenta la constante de proporcionalidad, disminuye el valor de $t_{p}$, es decir, del intervalo de mantenimiento preventivo a un costo mínimo. 


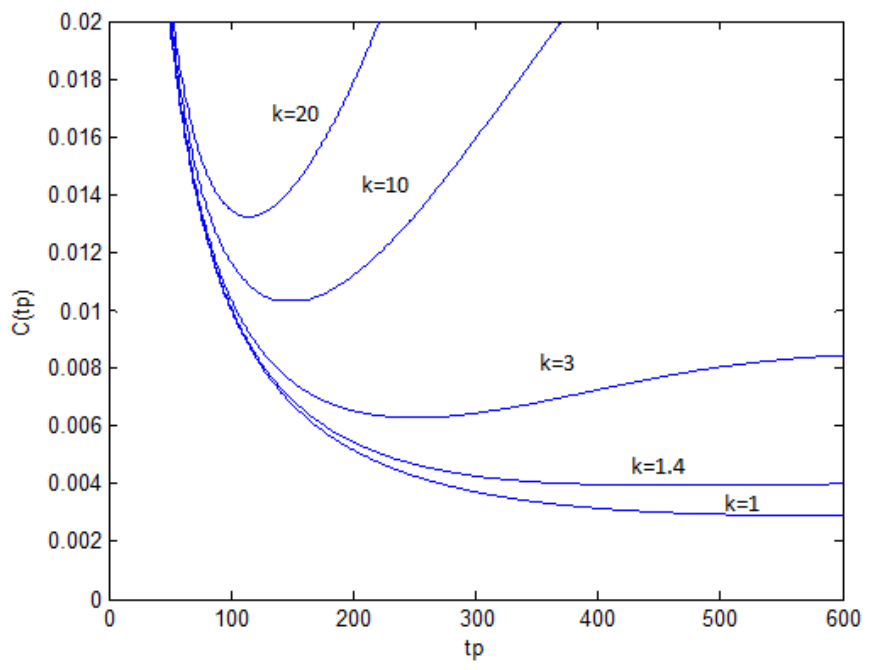

Figura 3.2: Costo esperado por unidad de tiempo

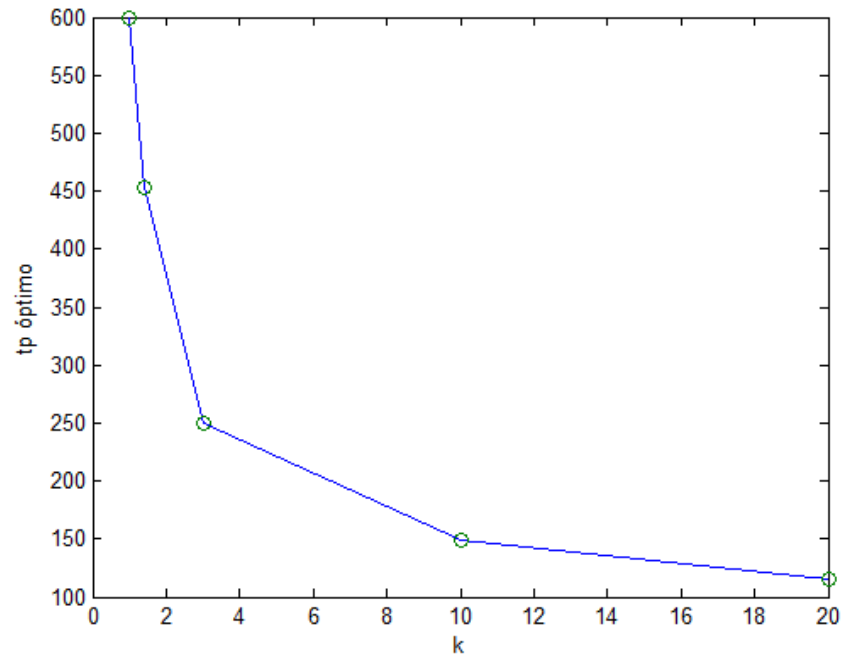

Figura 3.3: Relación entre el valor de k y el intervalo de mantenimiento $t_{p}$ óptimo.

En resumen, la política de mantenimiento preventivo basado en la edad, sugiere que se efectúe el reemplazo del componente cuando ocurre la falla o una vez que el componente haya alcanzado una edad de operación $t_{p}$, lo que ocurra primero. 


\subsection{Política II. Mantenimiento preventivo a intervalos constantes}

Esta política considera que se realizan reemplazos preventivos a intervalos constantes, independientemente del número de fallas intermedias (que también tienen un costo). En caso de que ocurra una falla antes de $t_{p}$, se realiza mantenimiento por falla.

De la misma forma que en la política anterior, la longitud del intervalo de tiempo (o edad) en el cual el sistema es reemplazado depende, entre otros, de los siguientes factores:

- Distribución del tiempo de falla.

- Costos de reemplazo por falla.

- Costos de reemplazo preventivo.

- Tiempo de inactividad por falla y reemplazo preventivo.

- Medida de efectividad: minimizar costos, maximizar disponibilidad, o lograr cierta confiabilidad.

Se supone que el tiempo considerado para realizar reemplazos preventivos y por falla es nulo y que los costos de mantenimiento preventivo son menores que los costos de mantenimiento por falla. Este modelo podrá implementarse sólo en la etapa de desgaste del componente, en la cual la tasa de falla es creciente.

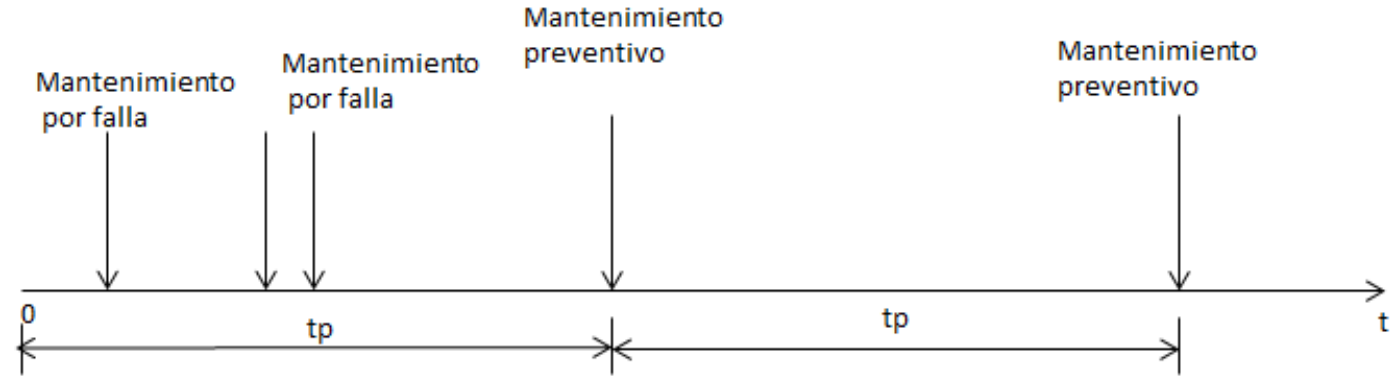

Figura 3.4: Reemplazo a intervalos constantes.

Nuevamente, el objetivo es encontrar el intervalo óptimo entre reemplazos preventivos, el cual minimiza el costo total esperado por unidad de tiempo. 
El costo total, generado durante un ciclo, está dado por

$$
C=\sum_{i=1}^{N\left(t_{p}\right)} C_{f i}+C_{p}
$$

$N\left(t_{p}\right)$ es una variable aleatoria que mide el número de fallas que ocurren en el intervalo $\left(0, t_{p}\right]$. De ahí que el conjunto de variables aleatorias $\left\{N\left(t_{p}\right), t_{p} \geq 0\right\}$ es un proceso de conteo que describe un Proceso no homogéneo de Poisson (NHPP) con función de intensidad $h\left(t_{p}\right), t_{p} \geq 0$ [8].

Por definición se tiene que el proceso de conteo $\{N(t), t \geq 0\}$ describe un NHPP con función de intensidad $h(t), \quad t \geq 0$ si satisface las siguientes propiedades:

a) $\mathrm{N}(0)=0$

b) El proceso tiene incrementos independientes, es decir, si $0 \leq t_{1}<t_{2}<t_{3}<t_{4}$, entonces las variables aleatorias $\left.\left[N\left(t_{2}\right)-N\left(t_{1}\right)\right)\right] \mathrm{y}$ $\left.\left[N\left(t_{4}\right)-N\left(t_{3}\right)\right)\right]$ son independientes.

c) $P\{N(t+\delta t)-N(t)=1\}=h(t) \delta t+o(\delta t), \quad \delta t \rightarrow 0$

d) $P\{N(t+\delta t)-N(t) \geq 2\}=o(\delta t), \quad \delta t \rightarrow 0$

En un Proceso de Poisson Homogéneo (NPP) la función de intensidad $h(t)$, a diferencia de la función de intensidad del NHPP que depende del tiempo, ahora es una función constante. Esto significa que si el Proceso de Poisson es homogéneo, con función de intensidad constante $\rho$, la distribución del número de fallas que ocurren en un intervalo de longitud $t$ es Poisson con media $\rho t$.

De las propiedades $c$ ) $y d$ ) se tiene que

$$
E[N(t, t+\delta t)]=\sum_{n=0}^{\infty} n P\{N(t, t+\delta t)=n\}=h(t) \delta t+o(\delta t), \quad \delta t \rightarrow 0
$$

Entonces, el número esperado de fallas en el intervalo de longitud $t_{p}$ está dado por

$$
\begin{aligned}
E\left[N\left(t_{p}\right)\right] & =E\left[\int_{0}^{t_{p}} N(t, t+d t)\right] \\
& =\int_{0}^{t_{p}} E[N(t, t+d t)] \\
& =\int_{0}^{t_{p}} h(t) d t \\
& =H\left(t_{p}\right)
\end{aligned}
$$


Por lo expuesto anteriormente, el costo total esperado por ciclo es

$$
C_{p}+C_{f} H\left(t_{p}\right)
$$

Inicialmente el largo del ciclo es $D=t_{p}+d_{p}$; pero como nuevamente $d_{p}=0$, el largo esperado del ciclo es simplemente $t_{p}$, el intervalo de mantenimiento preventivo. Por lo tanto, el costo esperado por unidad de tiempo para un intervalo de reemplazo $t_{p}$ es definido por la siguiente función de costo

$$
C\left(t_{p}\right)=\frac{C_{p}+C_{f} H\left(t_{p}\right)}{t_{p}}, t_{p} \in(0, \infty)
$$

Esto significa que el costo esperado por unidad de tiempo para un intervalo de reemplazo $t_{p}$ es la razón entre la suma del costo de mantenimiento preventivo más el costo de mantenimiento por falla multiplicado por el número esperado de fallas en el intervalo de reemplazo, y la longitud del intervalo, que es $t_{p}$.

Sabemos que el valor de $t_{p}$ que minimiza el costo total esperado por unidad de tiempo es aquel que satisface

$$
\begin{gathered}
\frac{d C\left(t_{p}\right)}{d t}=0 \\
\frac{d C\left(t_{p}\right)}{d t}=\frac{t_{p} C_{f} H^{\prime}\left(t_{p}\right)-C_{p}-C_{f} H\left(t_{p}\right)}{t_{p}^{2}}
\end{gathered}
$$

Si igualamos la derivada a cero tenemos

$$
\begin{aligned}
t p C_{f} H^{\prime}\left(t_{p}\right) & =C_{p}+C_{f} H\left(t_{p}\right) \\
t_{p} h\left(t_{p}\right) & =\frac{C_{p}}{C_{f}}+H\left(t_{p}\right) \\
t_{p} h\left(t_{p}\right)-H\left(t_{p}\right) & =\frac{C_{p}}{C_{f}}
\end{aligned}
$$

Esta última ecuación indica que nuevamente el intervalo óptimo de mantenimiento preventivo depende de la relación existente entre los costos de mantenimiento preventivo y por falla.

Ejemplo 3.2.1. En el caso de la distribución exponencial, para la cual la tasa de de fallas es constante, realizar mantenimiento preventivo genera un gasto extra, pues 
en este caso la probabilidad de falla es la misma para cualquier instante independientemente del tiempo que lleve funcionando el componente. Esto se ve en la solución del tiempo de mantenimiento preventivo óptimo, que es $t_{p}=\infty$.

Para esta distribución

$$
H\left(t_{p}\right)=\int_{0}^{t_{p}} \lambda d t=\lambda t_{p}
$$

Entonces el costo total esperado por unidad de tiempo queda como sigue:

$$
C\left(t_{p}\right)=\frac{C_{p}+C_{f} \lambda t_{p}}{t_{p}}, t_{p} \in(0, \infty)
$$

Luego

$$
\begin{aligned}
C^{\prime}\left(t_{p}\right) & =\frac{t_{p} C_{f} \lambda-C_{p}-t_{p} C_{f} \lambda}{t_{p}^{2}} \\
& =\frac{C_{p}}{t_{p}^{2}}
\end{aligned}
$$

Nos interesa el valor de $t_{p}$ para el cual

$$
\frac{C_{p}}{t_{p}^{2}}=0
$$

Esta última ecuación indica que $t_{p}=\infty$, lo cual significa que el mantenimiento preventivo se realiza una vez que ocurre la falla.

Ejemplo 3.2.2. Consideremos nuevamente el componente con tiempos de falla de distribución Weibull, cuyos parámetros son $\lambda=0.0025$ y $\beta=2.93$. Haremos uso de esta nueva política para realizar el análisis del comportamiento del valor que indica el intervalo de mantenimiento para costo mínimo, a partir de la relación que existe entre los costos de mantenimiento preventivo y por falla.

A continuación se describe la función del costo total esperado por unidad de tiempo, para un intervalo de reemplazo $t_{p}$, en el caso de la distribución Weibull.

Recordemos que la tasa de fallas está descrita por

$$
h(t)=\lambda \beta(\lambda t)^{\beta-1}, \quad t \geq 0
$$


Entonces el número esperado de fallas $H\left(t_{p}\right)$ nos queda como

$$
\begin{aligned}
H\left(t_{p}\right) & =\int_{0}^{t_{p}} \lambda \beta(\lambda t)^{\beta-1} d t \\
& =\left(\lambda t_{p}\right)^{\beta}
\end{aligned}
$$

Para la distribución Weibull, el número esperado de fallas en el intervalo $\left(0, t_{p}\right]$ es una potencia de $\lambda$ veces la longitud del intervalo. La potencia viene a ser el parámetro de forma de la distribución.

Según (3.8)

$$
C\left(t_{p}\right)=\frac{C_{p}+C_{f}\left(\lambda t_{p}\right)^{\beta}}{t_{p}}, t_{p} \in(0, \infty)
$$

En la Figura 3.5 se muestran la gráficas de la función del costo esperado por unidad de tiempo considerando los parámetros $C_{p}=1$ y $C_{f}=k C_{p}$. Nuevamente los valores de la constante $k$ se asignan de tal forma que los costos de mantenimiento por falla aumenten y que $C_{p}<k C_{f}$. En las gráficas se puede observar que a diferencia de la política de reemplazo basado en la edad, en este caso sí se tiene un minimo para valores $1 \leq k<1.4$.

En la Figura 3.6 se muestra nuevamente la relación entre la constante de proporcionalidad $k$ y el intervalo de reemplazo $t_{p}$ a costo minimo. Se puede observar que el intervalo óptimo de mantenimiento preventivo disminuye a medida que crece la constante de proporcionalidad $k$.

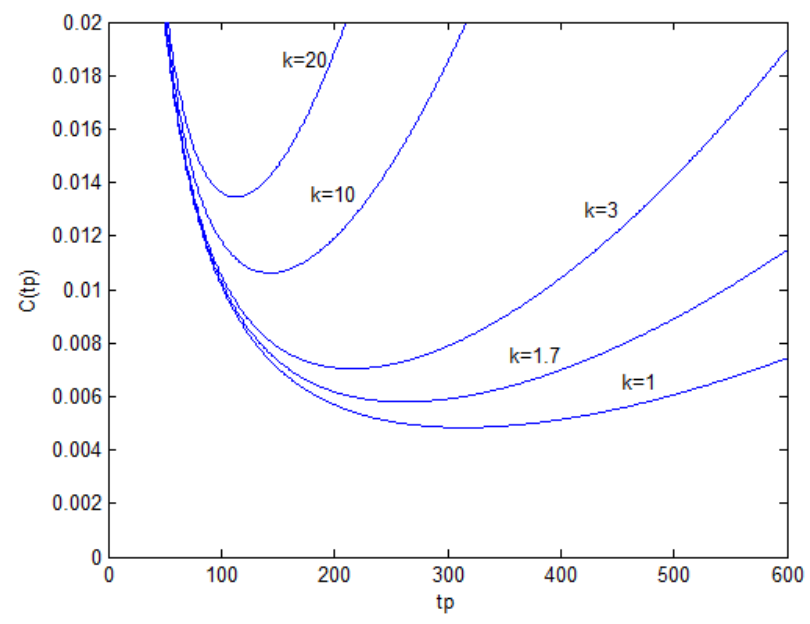

Figura 3.5: Costo esperado por unidad de tiempo 


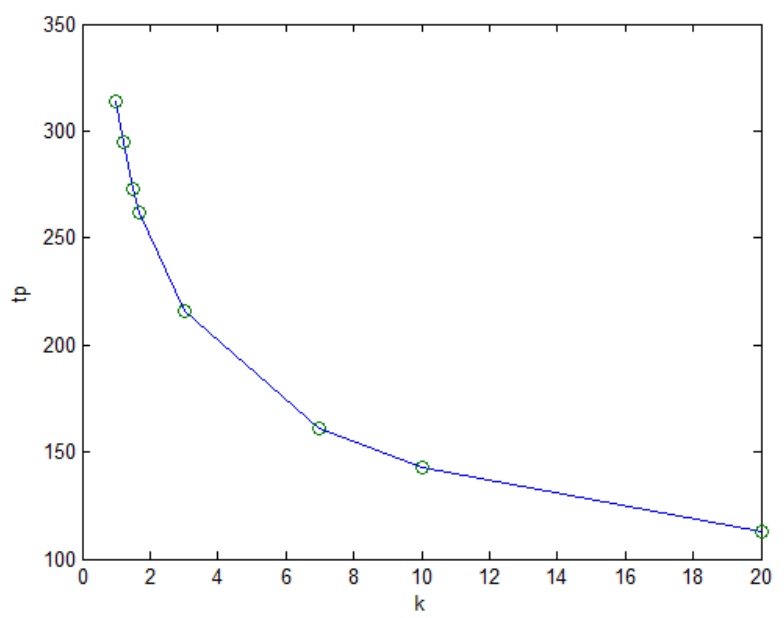

Figura 3.6: Relación entre el valor de k y el intervalo de mantenimiento $t_{p}$ óptimo.

\begin{tabular}{|c|c|c|}
\hline$k$ & Reemplazo a intervalos constantes \\
$C_{f}=k C_{p}$ & $\left(t_{p}, C\left(t_{p}\right)\right)$ & $\begin{array}{c}\text { Reemplazo por edad } \\
\left(t_{p}, C\left(t_{p}\right)\right)\end{array}$ \\
\hline 1 & $(314,0.0048)$ & $(\infty, 0.0029)$ \\
1.2 & $(295,0.0052)$ & $(\infty, 0.0034)$ \\
1.5 & $(273,0.0056)$ & $(416,0.0042)$ \\
1.7 & $(262,0.0058)$ & $(366,0.0046)$ \\
3 & $(216,0.0070)$ & $(250,0.0063)$ \\
7 & $(161,0.0094)$ & $(171,0.0090)$ \\
10 & $(143,0.0106)$ & $(149,0.0103)$ \\
20 & $(113,0.0135)$ & $(115,0.0132)$ \\
\hline
\end{tabular}

En la tabla anterior podemos observar que la política de reemplazo por edad genera menos reemplazos que la política de reemplazo a intervalos constantes. De hecho cuando $k=1.2$ mientras que la política de reemplazo a intervalos constantes presenta un óptimo en $t_{p}=295$, la política de reemplazo por edad sugiere que el reemplazo preventivo se realize hasta que ocurra la falla.

En resumen, la política de mantenimiento preventivo a intervalos constantes indica que el mantenimiento preventivo del componente se debe realizar en los instantes $t_{p}, 2 t_{p}, 3 t_{p}, \ldots$, independientemente del número de fallas intermedias. 


\subsection{Aplicación a un caso real}

En lo que sigue, vamos a realizar un análisis del mantenimiento preventivo del elevador del Edificio AT, ubicado en la Universidad Autónoma Metropolitana-Unidad Iztapalapa, en el cual se tiene programado un mantenimiento preventivo cada 30 días. Se aplicarán las políticas de reemplazo por edad e intervalos constantes para comparar el intervalo de reemplazo preventivo indicado por cada una de estas políticas con el intervalo de mantenimiento preventivo que tiene considerado el departamento de mantenimiento de la universidad.

El paro del funcionamiento del elevador del Edifico AT, es ocasionado principalmente por dos tipos de fallas: falla en los botones de llamada y puertas de cabina trabadas. Independientemente del mantenimiento preventivo que se tiene programado cada 30 días, cuando aparece una falla en el elevador, éste deja de funcionar y entonces se lleva a cabo un mantenimiento correctivo, con la finalidad de poner nuevamente en funcionamiento el elevador. Los costos estimados de mantenimiento preventivo y correctivo son $\$ 400.00$ y $\$ 1050.00$ respectivamente.

En la siguiente tabla se muestran los tiempos de falla del elevador ocurridos durante el periodo Enero 2012- Abril 2013.

Cuadro 3.1: Tiempos entre fallas, en días

\begin{tabular}{|c|c|c|c|c|c|c|c|}
\hline 5 & 7 & 7 & 9 & 13 & 14 & 15 & 16 \\
\hline 19 & 20 & 21 & 22 & 25 & 28 & 29 & 30 \\
\hline
\end{tabular}

Con ayuda del programa estadístico MINITAB, realizamos un ajuste paramétrico de las distribuciones Weibull y Normal a los tiempos de falla, para ver cuál de la dos distribuciones es la que mejor se ajusta a los datos.

En la Figura 3.7 se muestra el gráfico de probabilidad de los tiempos de falla correspondiente a la distribución Weibull. Los parámetros estimados de la distribución son: $\lambda=19.78$ y $\beta=2.4273$. El gráfico de probabilidad de los tiempos de falla correspondiente a la distribución Normal se muestra en la Figura 3.8, los parámetros estimados son: $\mu=17.5$ y $\sigma=7.85$.

Podemos observar que los tiempos de falla pueden ser modelados tanto por la distribución Weibull como por la distribución Normal. De hecho el estadístico AndersonDarling (que se muestra en las Figuras 3.7 y 3.8 como AD*) nos da una medida de lo alejados que se encuentran los tiempos de falla de la recta que representa a cada 


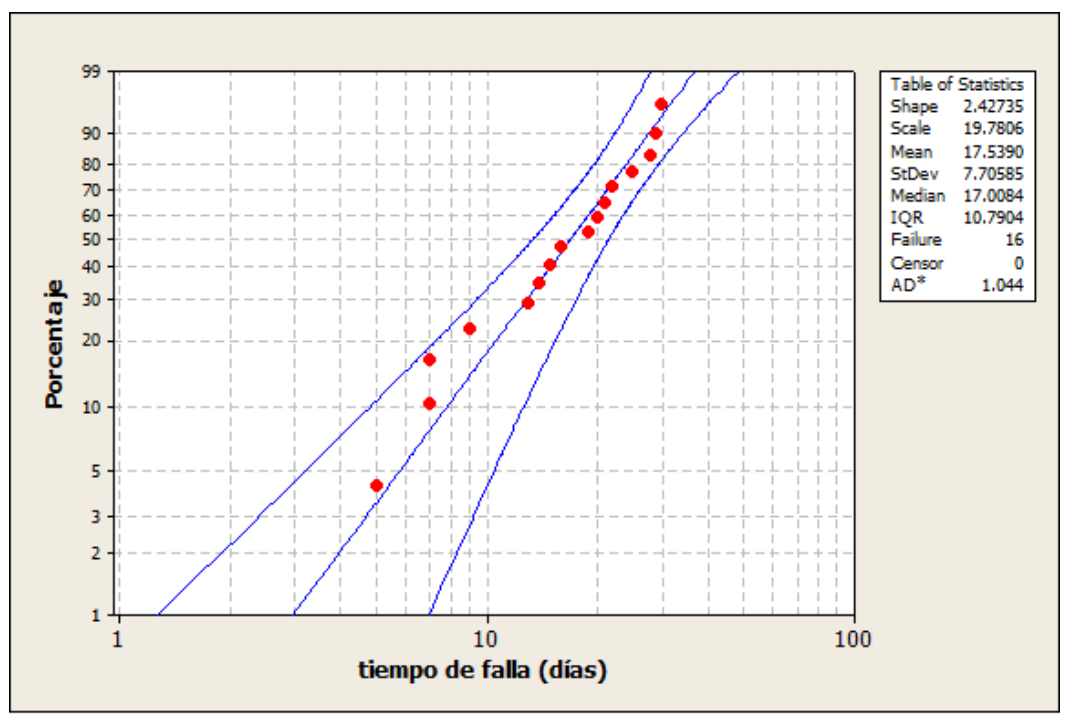

Figura 3.7: Gráfico de probabilidad para tiempos de falla del elevador.Distribución Weibull.

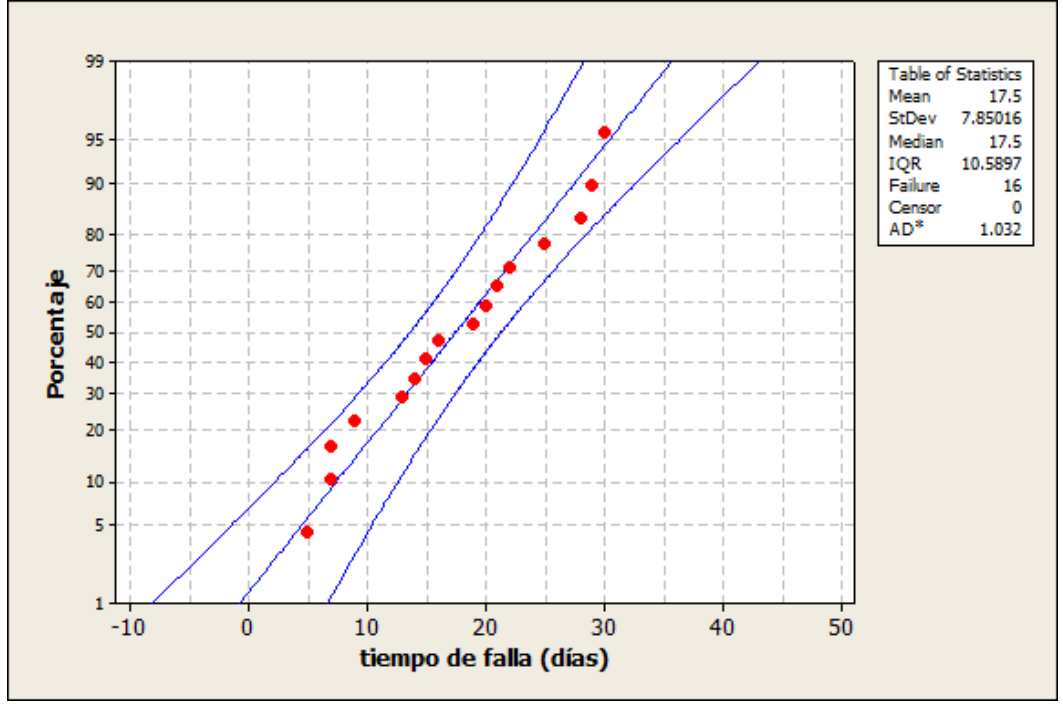

Figura 3.8: Gráfico de probabilidad para tiempos de falla del elevador. Distribución Normal.

distribución. Cuanto mejor sea el ajuste, menor será el valor de dicho estadístico. Los valores del estadístico son 1.044 para la distribución Weibull y 1.032 para la distribución normal.

Ahora veamos como se comporta la tasa de falla para los tiempos de falla del elevador. En la Figura 3.9 se muestra la tasa de falla para cada distribución. Observe 
que para ambos casos la tasa de falla es creciente, pero después de cierto tiempo ésta crece más rápido en el caso de la distribución Normal.

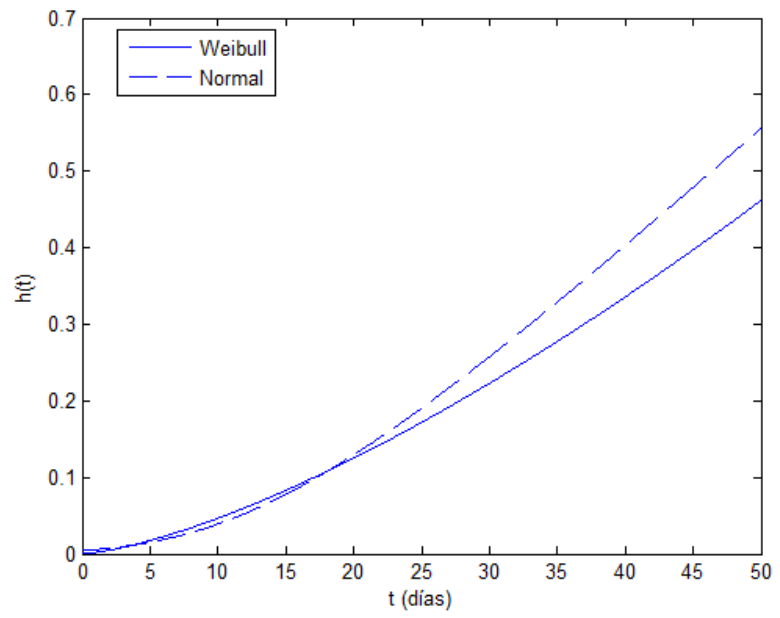

Figura 3.9: Tasa de falla para las distribuciones Normal y Weibull.

Cuadro 3.2: Distribución Weibull

\begin{tabular}{|c|c|c|}
\hline $\begin{array}{c}\text { Política } \\
\text { de reemplazo }\end{array}$ & $\begin{array}{c}\text { Intervalo de reemplazo óptimo } \\
\text { (días) }\end{array}$ & $\begin{array}{c}\text { Costo mínimo } \\
(\$ / \text { día })\end{array}$ \\
\hline Edad & 14 & 50.48 \\
Intervalos constantes & 11 & 59.30 \\
\hline
\end{tabular}

Cuadro 3.3: Distribución Normal

\begin{tabular}{|c|c|c|}
\hline $\begin{array}{c}\text { Política } \\
\text { de reemplazo }\end{array}$ & $\begin{array}{c}\text { Intervalo de reemplazo óptimo } \\
(\text { días })\end{array}$ & $\begin{array}{c}\text { Costo mínimo } \\
(\$ / \text { día })\end{array}$ \\
\hline Edad & 15 & 48.98 \\
Intervalos constantes & 12 & 57.52 \\
\hline
\end{tabular}

En los cuadros 3.2 y 3.3 se muestran los resultados obtenidos para el intervalo óptimo de reemplazo preventivo y el costo mínimo en cada política de reemplazo y para cada distribución. Se puede observar que cuando se supone que los tiempos de falla son modelados por la distribución Weibull, la diferencia entre los intervalos de reemplazo óptimo es 3 días. Como es de esperarse, el costo mínimo es más grande en la política de reemplazo a intervalos constantes. El mismo comportamiento se tiene cuando los tiempos de falla son modelados por la distribución Normal. 
En las Figuras 3.10 y 3.11 se muestran las gráficas del costo esperado por unidad de tiempo para las dos políticas de reemplazo, según sea la distribución Weibull o Normal.

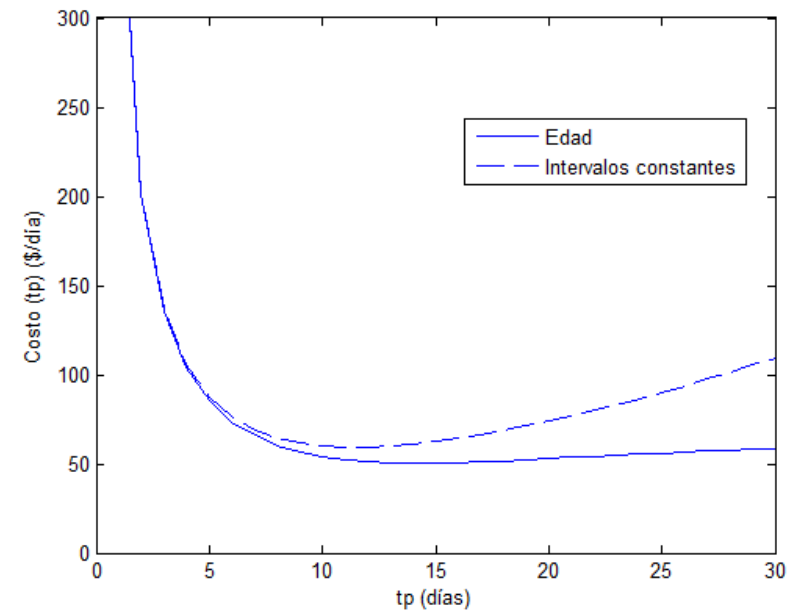

Figura 3.10: Costo esperado por unidad de tiempo. Distribución Weibull

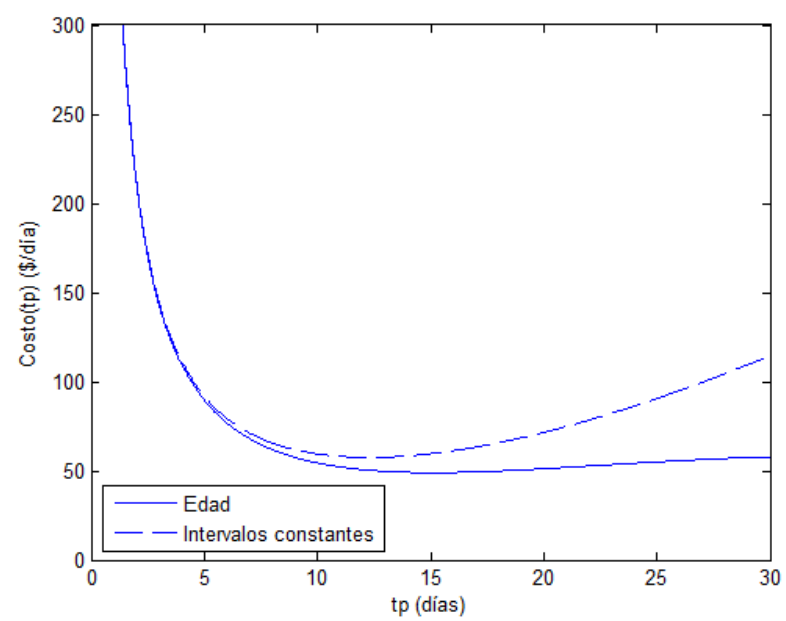

Figura 3.11: Costo esperado por unidad de tiempo. Distribución Normal

En el Cuadro 3.4 se muestran los costos que se derivan del plan actual de mantenimiento del elevador. Estos costos se calcularon evaluando la función del costo esperado por unidad de tiempo correspondiente a la política de mantenimiento basado en la edad, para un valor de $t=30$ días. 
Cuadro 3.4: Plan de mantenimiento actual

\begin{tabular}{|c|c|c|}
\hline $\begin{array}{c}\text { Reemplazo por } \\
\text { edad }\end{array}$ & $\begin{array}{c}\text { Intervalo de reemplazo } \\
\text { (días) }\end{array}$ & $\begin{array}{c}\text { Costo } \\
(\$ / \text { día })\end{array}$ \\
\hline Weibul & 30 & 58.31 \\
Normal & 30 & 57.89 \\
\hline
\end{tabular}

En lo que que se refiere a qué política de reemplazo habría que elegir, nuevamente optamos por elegir la política de reemplazo por edad, que es la que indica el intervalo de reemplazo más grande para cualquiera de las distribuciones. Podemos observar que el intervalo más grande, 15 días, se obtiene cuando los tiempos de falla están normalmente distribuidos. Por otro lado, ¿qué función de distribución es la que vamos a utilizar? Hemos visto que el mejor resultado, 15 días con un costo mínimo de $\$ 48.98$, se obtiene con la distribución Normal; no obstante, computacionalmente con la distribución Weibull se requieren menos cálculos y tiempo para obtener los resultados que con la distribución Normal.

Con la aplicación de estas políticas se ha logrado optimizar, bajo ciertas suposiciones, los costos de mantenimiento preventivo del elevador. ¿Qué sucede con los costos de mantenimiento del plan actual? La política de reemplazo basado en la edad indica que el costo que se genera con el actual intervalo de mantenimiento preventivo, 30 días, es de $\$ 58.31$ en el caso de la distribución Weibull y $\$ 57.89$ para la distribución normal. Nótese que ambos costos son más altos que los que sugiere la política de mantenimiento por edad ( $\$ 50.48$ y $\$ 48.98$ respectivamente).

Por otro lado, hemos hablado de que el mantenimiento preventivo tiene por objetivo aumentar la confiabilidad de un equipo o sistema, y por ende, disminuir la tasa de falla del sistema. En lo que sigue vamos a calcular el $M T B F$ del elevador cuando se consideran actividades de mantenimiento preventivo programadas cada 14 días para después compararlo con el $M T B F$ que se obtiene cuando se lleva a cabo un mantenimiento programado cada 30 días.

En la sección 2.4 estudiamos la función de densidad que resulta cuando se practican actividades de mantenimiento preventivo. Se dedujo que la función resultante es de tipo exponencial.

De manera general, la función de densidad de falla que se tiene después del mantenimiento preventivo está descrita por

$$
f_{M P}(t)= \begin{cases}\sum_{k=0}^{\infty} f_{k}(t), & \text { si } k t_{p}<t<(k+1) t_{p} \\ 0, & \text { de otra forma. }\end{cases}
$$

Si consideramos el caso en el que los tiempos de falla del elevador son modelados 
por la distribución Weibull entonces

$$
f_{k}(t)=\sum_{k=0}^{\infty}\left[e^{-\left(\frac{1}{\lambda} t_{p}\right)^{\beta}}\right]^{k} \frac{\beta}{\lambda}\left(\frac{1}{\lambda}\left(t-k t_{p}\right)\right)^{\beta-1} e^{-\left(\frac{1}{\lambda}\left(t-k t_{p}\right)\right)^{\beta}}
$$

En nuestro caso $t_{p}=14$ días, $\lambda=19.78$ y $\beta=2.4273$. Por tanto

$$
f_{M P}(t)= \begin{cases}\sum_{k=0}^{\infty} 0.1227\left[e^{-(0.7583)^{2.4273}}\right]^{k}(0.05 t)^{1.4273} e^{-(0.05 t)^{2.4273}}, & \text { si } k t_{p}<t<(k+1) t_{p} \\ 0, & \text { de otra forma } .\end{cases}
$$

es la función de densidad de fallo con mantenimiento preventivo. Con esto se tiene que

$$
M T B F=\int_{0}^{\infty} t f_{M P}(t) d t
$$

es el tiempo medio entre fallas con mantenimiento preventivo. Esta expresión se resuelve numéricamente. Una aproximación que ha funcionado en aplicaciones se basa en la tasa de falla sin mantenimiento preventivo. Como $f_{M P}(\cdot)$ es de tipo exponencial, el $M T B F$ con mantenimiento preventivo se puede aproximar usando la expresión $1 / h^{*}(t)$, donde $h^{*}(t)$ es el valor medio de $h(t)$ en el intervalo $\left[0, t_{p}\right]$, siendo $t_{p}$ la longitud del intervalo de mantenimiento preventivo.

La tasa de falla en el caso de la distribución Weibull es

$$
h(t)=\frac{\beta}{\lambda}\left(\frac{t}{\lambda}\right)^{\beta-1}, t \geq 0
$$

Si sustituimos los valores de los parámetros $\lambda$ y $\beta$ tenemos que la tasa de falla del elevador para cada valor de $t$ es

$$
h(t)=0.1227(0.05 t)^{1.4273} .
$$

Ahora calculamos el valor medio de $h(t)$ en el intervalo $[0,14]$

$$
\begin{aligned}
h^{*}(t) & =\frac{1}{14} \int_{0}^{14} 0.1237(0.05 t)^{1.4272} d t \\
& =0.0306
\end{aligned}
$$

De esta última expresión se tiene que $M T B F=32.68$ es el valor del tiempo medio entre fallas con mantenimiento preventivo cada 14 días. Hemos visto entonces como las actividades de mantenimiento preventivo programadas cada 14 días, según la política de reemplazo basado en la edad, mejoran el MTBF del elevador, pasando de 17.54 días, que es la media de la distribución Weibull (ver Figura 3.7) a 32.68 días si se realiza el mantenimiento preventivo. 
Podemos concluir que el tipo de mantenimiento que resulta óptimo es el de edad a 14 días con un costo de $\$ 50.48$ que es el mínimo. El tiempo medio entre fallas es de 32.68 días, el cual es mejor que el que se tenía con mantenimiento programado cada 30 días con un costo de $\$ 58.31$. 
$\overline{C a p i ́ t u l o ~}_{1}$

\section{Políticas óptimas de mantenimiento preventivo para sistemas con varios componentes.}

En el capítulo anterior se estudiaron las políticas de reemplazo para un sólo componente. Sin embargo, los sistemas pueden estar formados por dos o más componentes, los cuales pueden estar organizados en distintas configuraciones: sistema en serie, sistema paralelo o sistema mixto; esto según la complejidad del sistema. En este capítulo se expone la generalización de las políticas de reemplazo por edad e intervalos constantes para sistemas con varios componentes.

Un sistema se define como un conjunto de componentes relacionados entre sí para llevar a cabo una misión conjunta. Se supone que estos componentes fallan de manera independiente unos de otros.

Los sistemas pueden clasificarse en reparables y no reparables. Los sistemas reparables son aquellos que después de fallar en realizar al menos una de sus funciones requeridas pueden ser restaurados para realizar todas sus funciones por cualquier otro método que no sea el reemplazo del sistema completo. Los sistemas no reparables implican un cambio completo; estos últimos sistemas serán el objeto de nuestro estudio. 


\subsection{Confiabilidad de sistemas en serie}

Se considera que un sistema tiene componentes que están colocados en serie cuando la falla de uno o más componentes antes de terminar el proceso origina la falla de todo el sistema. El tiempo de vida de un sistema en serie es igual al tiempo de vida más pequeño entre todos los componentes del sistema, es decir, el sistema en serie sólo puede durar tanto como su componente más frágil. La confiabilidad de un sistema en serie es menor o igual que la confiabilidad del componente menos confiable. El diagrama de bloques de un sistema en serie con $n$ componentes es mostrado en la Fig. 4.1

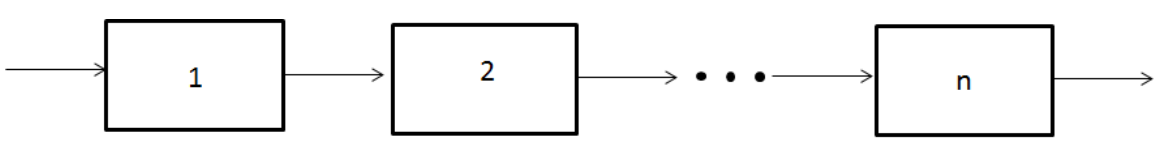

Figura 4.1: Diagrama de bloques de un sistema en serie con $n$ componentes

Supóngase una serie de $n$ componentes que trabajan de forma independiente. Sea $P_{i}$ la probabilidad de que el componente $i$ funcione. Si $P_{s s}$ denota la probabilidad de que el sistema funcione, entonces

$$
P_{\text {ss }}(\text { funcione })=P_{1}(\text { funcione }) P_{2}(\text { funcione }) \cdots P_{n}(\text { funcione })
$$

Sean $F_{i}(t)=P_{i}(T \leq t)$ la función de distribución de la variable aleatoria $T$, que representa la longitud del tiempo que el componente $i$ funciona y falla en $t \mathrm{y}$ $R_{i}(t)=P(T>t)$ la probabilidad de que el componente $i$ sobreviva al tiempo $t$ (no falle en el intervalo $(0, \mathrm{t}])$.

De esta forma, la confiabilidad del sistema para cualquier instante $t$, es la probabilidad de que todos los componentes sobrevivan en $t$, por lo que

$$
R_{s s}(t)=R_{1}(t) R_{2}(t) \cdots R_{n}(t)=\prod_{i=1}^{n} R_{i}(t)
$$

En términos de la función de distribución podemos escribir

$$
F_{s}(t)=1-\prod_{i=1}^{n} R_{i}(t)=1-\prod_{i=1}^{n}\left(1-F_{i}(t)\right)
$$


Si los $n$ componentes que trabajan de manera independiente tienen la misma confiabilidad, es decir, $R_{i}(t)=R(t), \quad i=1,2, \cdots, n$ entonces

$$
R_{s s}(t)=[R(t)]^{n}
$$

Ejemplo 4.1.1. Considérese un sistema con dos componentes $A$ y $B$ conectados en serie, cuyos tiempos de falla son modelados por la distribución exponencial. Las tasas de falla para cada componente son 0.2 y 0.5 por 10,000 hrs. respectivamente. ¿Cuál es la confiabilidad del sistema para $t=20,000$ horas?

Recordemos que para componentes con tasa de falla constante, la función de confiabilidad es

$$
R(t)=e^{-\lambda t}
$$

Entonces para el componente $A, R_{A}(t)=e^{-0.2 t}$. De manera similar $R_{B}(t)=e^{-0.5 t}$.

Por Ecuación 4.1 se tiene que la confiabilidad del sistema en serie para cualquier instante $t$ es

$$
R_{s s}(t)=e^{-0.2 t} e^{-0.5 t}=e^{-0.7 t}
$$

Por lo tanto, la confiabilidad del sistema en $t=20,000$ horas es

$$
R_{s s}(2)=e^{-1.4}=0.2466
$$

Observe que la confiabilidad del sistema es menor que la de cualquiera de sus componentes.

\subsubsection{Tasa de falla del sistema}

La tasa de falla del sistema está dada por

$$
h_{s s}(t)=\sum_{i=1}^{n} h_{i}(t)
$$

siendo $h_{i}(t)$ la tasa de falla del componente $i$.

La ecuación 4.2 se puede obtener utilizando algunas de las relaciones que se mencionaron en el capítulo 1. La definición de la tasa de falla que se dio en la ecuación 1.7 es

$$
h_{i}(t)=-\frac{d}{d t} \ln \left(R_{i}(t)\right)
$$


Luego

$$
h_{s s}(t)=-\frac{d}{d t} \ln \left(R_{s s}(t)\right)
$$

Por lo que acabamos de mencionar, se tiene que

$$
-\ln R_{s s}(t)=-\ln \left(\prod_{i=1}^{n} R_{i}(t)\right)=\sum_{i=1}^{n}-\ln R_{i}(t)
$$

Entonces

$$
\begin{aligned}
-\frac{d}{d t} \ln R_{s s}(t) & =\frac{d}{d t} \sum_{i=1}^{n}-\ln R_{i}(t) \\
& =\sum_{i=1}^{n} \frac{d}{d t}\left(-\ln R_{i}(t)\right) \\
& =\sum_{i=1}^{n} h_{i}(t) .
\end{aligned}
$$

\subsubsection{Sistema en serie con componentes de distribu- ción exponencial}

Consideremos un sistema en serie con $n$ componentes para los cuales el tiempo de falla es modelado por la distribución exponencial. Sea $\lambda_{i}$ la tasa de falla del componente $i$.

La función de confiabilidad del componente $i$ está dada por

$$
R_{i}(t)=e^{-\lambda_{i} t}, \quad t \geq 0
$$

Entonces la función de confiabilidad del sistema en serie con distribución exponencial en cada componente está caracterizada por

$$
R_{s s}(t)=e^{-\lambda_{1} t} e^{-\lambda_{2} t} e^{-\lambda_{3} t} \cdots e^{-\lambda_{n} t}=e^{-\left(\sum_{i=1}^{n} \lambda_{i}\right) t}
$$

donde

$$
\sum_{i=1}^{n} \lambda_{i}=h_{s s}(t)
$$


Observemos que la tasa de falla de todo el sistema también es constante.

Ahora veamos qué sucede con el $M T B F_{s s}$ del sistema. De (1.2) sabemos que

$$
M T B F_{s s}=\int_{0}^{\infty} R_{s s}(t) d t
$$

Para el sistema con componentes con distribución del tiempo de falla exponencial queda que

$$
\begin{aligned}
\operatorname{MTBF}_{s s} & =\int_{0}^{\infty} R_{s s}(t) d t \\
& =\int_{0}^{\infty} e^{-\left(\sum_{i=1}^{n} \lambda_{i}\right) t} d t \\
& =\int_{0}^{\infty} e^{-h_{s s} t} d t \\
& =\frac{1}{h_{s s}(t)}=\frac{1}{\sum_{i=1}^{n} \lambda_{i}}
\end{aligned}
$$

Cuando los componentes son i.i.d. con tasa de falla constante $\lambda$ se tienen las siguientes ecuaciones:

$$
\begin{gathered}
h_{s s}=n \lambda \\
R_{s s}(t)=e^{-n \lambda t}, \quad t \geq 0 \\
M T B F_{s s}=\frac{1}{n \lambda}
\end{gathered}
$$

\subsection{Política de reemplazo por edad para un sistema en serie}

Recordemos que el objetivo de los modelos de reemplazo es determinar el intervalo óptimo entre reemplazos sucesivos, el cual minimiza el costo total esperado por unidad de tiempo.

Ahora vamos a suponer que los $n$ componentes del sistema en serie son reemplazados de manera conjunta. Como en el caso de un sólo componente, los componentes son reemplazados en la falla del sistema o después de un cierto intervalo de tiempo 
$t_{p}$, lo que ocurra primero.

Nuevamente supondremos que después de cada reemplazo el sistema queda como nuevo, que los reemplazos son instantáneos, que los costos de mantenimiento preventivo son menores que los costos de mantenimiento por falla y que los componentes fallan de manera independiente.

La expresión del costo total esperado por unidad de tiempo sigue estando descrita por (3.1).

Ahora el costo esperado del ciclo (3.2) se escribe como

$$
C_{p} \prod_{i=1}^{n} R_{i}\left(t_{p}\right)+C_{f}\left[1-\prod_{i=1}^{n} R_{i}\left(t_{p}\right)\right]
$$

El largo esperado de un ciclo definido por (3.3) queda como

$$
\int_{0}^{t_{p}} R_{s s}(t) d t=\int_{0}^{t_{p}} \prod_{i=1}^{n} R_{i}(t) d t
$$

Finalmente, la expresión para el costo total esperado por unidad de tiempo para un sistema en serie está dado por

$$
C_{s s}\left(t_{p}\right)=\frac{C_{p} \prod_{i=1}^{n} R_{i}\left(t_{p}\right)+C_{f}\left[1-\prod_{i=1}^{n} R_{i}\left(t_{p}\right)\right]}{\int_{0}^{t_{p}} \prod_{i=1}^{n} R_{i}(t) d t}, t_{p} \geq 0
$$

$C_{p}$ y $C_{f}$ son los costos de mantenimiento preventivo y costo de mantenimiento por falla respectivamente.

Ejemplo 4.2.1. Supongamos un sistema en serie formado por 2 componentes idénticos, para las cuales el tiempo de falla es modelado por la distribución Weibull con parámetro de escala $\lambda_{i}=0.01$ y parámetro de forma $\beta_{i}=3.5, i=1,2$. Cada componente puede ser reemplazado con un costo de $\$ 3,000.00$, por lo que el costo de reemplazar el sistema es de $\$ 6,000.00$; pero si un componente falla el costo de mantenimiento por falla es de $\$ 10,000.00$. ¿Qué tan frecuente debería ser reemplazado el sistema?

Primero vamos a verificar que la tasa de falla del sistema es una función creciente.

Recordemos que, en la distribución Weibull, para cada componente i, la tasa de falla está dada por

$$
h_{i}(t)=\lambda_{i} \beta_{i}\left(\lambda_{i} t\right)^{\beta_{i}-1}, \quad t \geq 0, \quad i=1,2 .
$$


Entonces la tasa de falla del sistema es

$$
h_{s s}(t)=\sum_{i=1}^{2} \lambda_{i} \beta_{i}\left(\lambda_{i} t\right)^{\beta_{i}-1}
$$

Si sustituimos $\lambda_{i}=0.001$ y $\beta_{i}=3.5, \quad i=1,2$ nos queda que

$$
h_{s s}(t)=2[0.01(3.5)](0.01 t)^{2.5}=0.07(0.01 t)^{2.5}
$$

Podemos observar claramente que $h_{s s}(\cdot)$ es una función creciente.

Ahora vamos a escribir la expresión del costo total esperado por unidad de tiempo, para cualquier instante $t_{p} \geq 0$

La confiabilidad del sistema es

$$
R_{s s}\left(t_{p}\right)=\prod_{i=1}^{2} e^{-\left(0.01 t_{p}\right)^{3.5}}=e^{-2\left(0.01 t_{p}\right)^{3.5}}
$$

Por tanto

$$
C_{s s}\left(t_{p}\right)=\frac{6000 e^{-2\left(0.01 t_{p}\right)^{3.5}}+10000\left[1-e^{-2\left(0.01 t_{p}\right)^{3.5}}\right]}{\int_{0}^{t_{p}} e^{-2(0.01 t)^{3.5}} d t}
$$

Finalmente, el valor de $t_{p}$ que indica el intervalo de tiempo entre dos reemplazos sucesivos para los componentes del sistema a un costo minimo es $t_{p}=72$ unidades de tiempo, a un costo de $\$ 124.17$ por unidad de tiempo.

En la Figura 4.2 se muestra la curva del costo esperado por unidad de tiempo.

\subsection{Política de reemplazo a intervalos constantes para un sistema en serie}

En esta política se supone que los componentes del sistema son reemplazados de manera conjunta después de que los componentes han alcanzado una edad de operación $t_{p}$ y cuando fallan. Al igual que en la política anterior supondremos que después de cada reemplazo el sistema queda como nuevo, que los reemplazos son 


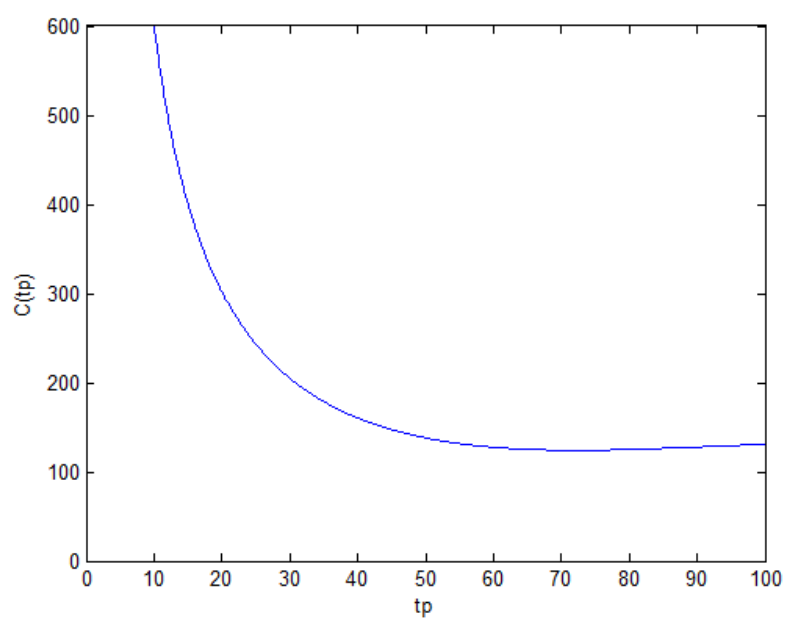

Figura 4.2: Costo esperado por unidad de tiempo para un sistema en serie con dos componentes Weibull, según la política de reemplazo por edad.

instantáneos y que los componentes fallan de manera independiente.

Para esta política, el largo esperado de un ciclo es $t_{p}$.

El costo total, generado durante un ciclo (3.6), está dado por

$$
C_{p}+C_{f} H_{s s}\left(t_{p}\right)
$$

Donde $H_{s s}\left(t_{p}\right)=\int_{0}^{t_{p}} h_{s s}(t) d t$ es el número esperado de fallas en el intervalo $\left(0, t_{p}\right)$.

Finalmente, la expresión para el costo total esperado por unidad de tiempo para un sistema en serie está dado por

$$
C_{s s}\left(t_{p}\right)=\frac{C_{p}+C_{f} H_{s s}\left(t_{p}\right)}{t_{p}}, t_{p}>0
$$

$C_{p}$ y $C_{f}$ son los costos de mantenimiento preventivo y costo de mantenimiento por falla respectivamente.

Ejemplo 4.3.1. Consideremos nuevamente el sistema en serie del ejemplo anterior. Si se considera la política de reemplazo a intervalos constantes, ¿qué tan frecuente deberían ser reemplazados los componentes del sistema?

Vamos a calcular $H_{s s}(t)$, el número esperado de fallas en el intervalo $\left(0, t_{p}\right)$. 
Sabemos que $h_{s s}(t)=0.07(0.01 t)^{2.5}$

Entonces

$$
\begin{aligned}
H_{s s}(t) & =\int_{0}^{t_{p}} h_{s s}(t) d t \\
& =\int_{0}^{t_{p}} 0.07(0.01 t)^{2.5} d t=2 X 10^{-7} t_{p}^{3.5}
\end{aligned}
$$

Por tanto, la expresión del costo total esperado por unidad de tiempo se escribe como

$$
C\left(t_{p}\right)=\frac{6000+0.0020 t_{p}^{3.5}}{t_{p}}, \quad t_{p}>0
$$

En la Figura 4.3 se muestra la curva que representa el costo total esperado por unidad de tiempo para esta nueva política.

El tiempo óptimo de mantenimiento preventivo es $t_{p}=55$ unidades de tiempo, con un costo mínimo de $\$ 153.96$ por unidad de tiempo.

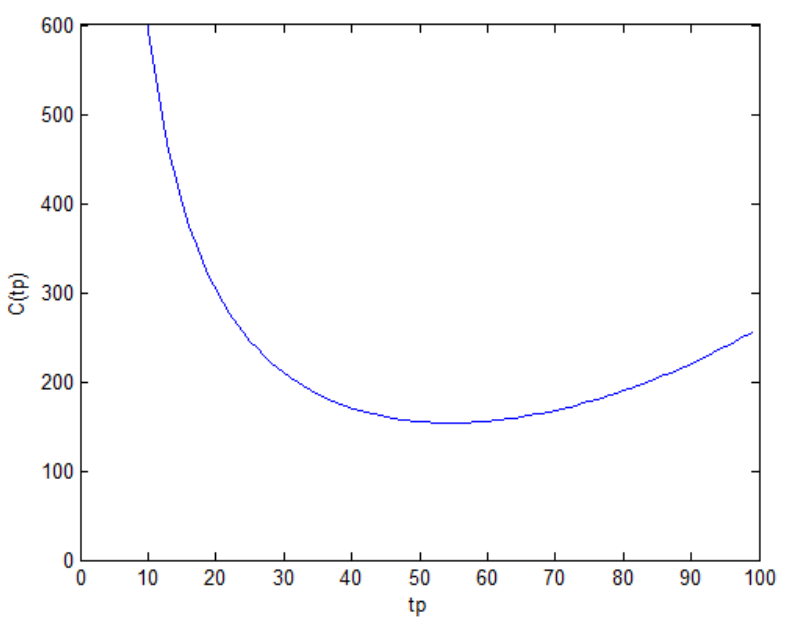

Figura 4.3: Costo esperado por unidad de tiempo para un sistema en serie con dos componentes Weibull, según la política de reemplazo a intervalos constantes

Nótese que la política de reemplazo a intervalos constantes da un óptimo con tiempos de reemplazo menores que la política de reemplazo por edad y por ello, mayor costo en el óptimo. Por esta razón conviene usar la política de reemplazo por edad cuando la naturaleza de los componentes lo permitan. 


\subsection{Confiabilidad de sistemas en paralelo}

Se dice que un sistema tiene componentes que están en paralelo cuando solamente la falla de todas las componentes en el sistema origina la falla del sistema. Es decir, para que un sistema en paralelo funcione, es suficiente que al menos uno de los componentes opere sin falla. El diagrama de bloques para un sistema en paralelo se muestra en la Figura 4.4

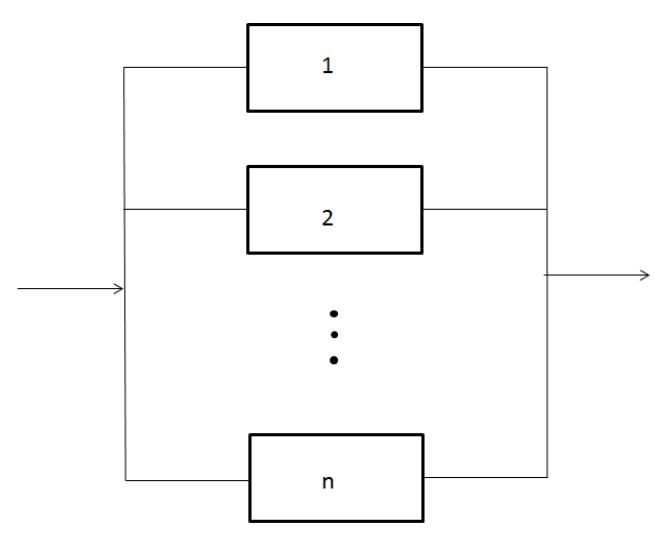

Figura 4.4: Diagrama de bloques de un sistema en paralelo con $n$ componentes

Supóngase $n$ componentes que trabajan de manera independiente, los cuales están colocados en paralelo. En este caso

$$
\begin{aligned}
P_{s p}(\text { nofuncione }) & =P_{1}(\text { nofuncione }) P_{2}(\text { nof uncione }) \cdots P_{n}(\text { nofuncione }) \\
& =\left(1-P_{1}(\text { funcione })\right)\left(1-P_{2}(\text { funcione })\right) \cdots\left(1-P_{n}(\text { funcione })\right)
\end{aligned}
$$

Entonces

$$
P_{s p}(\text { funcione })=1-P_{s p}(\text { nofuncione })
$$

De ahí que la confiabilidad del sistema $R_{s p}(t)$, está dada por

$$
R_{s p}(t)=1-\prod_{i=1}^{n}\left(1-R_{i}(t)\right), \quad t \geq 0
$$

Es decir, la confiabilidad del sistema en paralelo es uno menos el producto de la probabilidad de falla de cada uno de los componentes. 
Ejemplo 4.4.1. Supongamos ahora los dos componentes del Ejemplo 4.1.1, pero colocados en un sistema en paralelo. ¿Cuál es la confiabilidad del sistema para $t=20,000$ horas?

La confiabilidad del sistema en cualquier instante t está dada por

$$
\begin{aligned}
R_{s p}(t) & =1-\left[\left(1-e^{-0.2 t}\right)\left(1-e^{-0.5 t}\right)\right] \\
& =1-\left(1-e^{-0.5 t}-e^{-0.2 t}+e^{-0.7 t}\right) \\
& =e^{-0.5 t}+e^{-0.2 t}-e^{-0.7 t}
\end{aligned}
$$

Por lo tanto, la confiabilidad del sistema en $t=20,000$ horas es

$$
R_{s p}(2)=e^{-1}+e^{-0.4}-e^{-1.4}=0.7916
$$

Observemos que en este caso la confiabilidad del sistema es mayor que la confiabilidad de cualquiera de sus componentes.

Nuevamente, si suponemos que cada uno de los componentes del sistema tienen la misma confiabilidad, es decir, $R_{i}(t)=R(t), \quad i=1,2, \cdots, n$, entonces

$$
R_{s p}(t)=1-\prod_{i=1}^{n}(1-R(t))^{n}
$$

Recordemos también que la función de densidad de falla de acuerdo a (1.5) se escribe como

$$
f_{s p}(t)=-\frac{d R_{s p}}{d t}
$$

La tasa de falla del sistema, según (1.1), se escribe como

$$
h_{s p}(t)=\frac{f_{s p}}{R_{s p}}
$$

Por (1.2) el tiempo medio entre falla del sistema es

$$
M T B F_{s p}=\int_{0}^{\infty} R_{s p}(t) d t
$$

En un sistema en paralelo sólo se requiere que un componente funcione para hacer que el sistema funcione. El resto de los componentes incluidos en el sistema son llamados componentes redundantes. Éstos son incluidos para aumentar la probabilidad de tener al menos un componente trabajando. La redundancia es una técnica usada ampliamente en ingeniería para reforzar la confiabilidad del sistema. En Dodson (2002) 9], se pueden estudiar sistemas más complejos, tales como los sistemas $k$ de $n$, sistemas de $n$ componentes que son capaces de funcionar correctamente si al menos $k$ de los componentes operan correctamente. 


\subsubsection{Sistema en paralelo con componentes de dis- tribución exponencial}

Consideremos un sistema en paralelo con $n$ componentes para los cuales el tiempo de falla es modelado por la distribución exponencial. Sea $\lambda_{i}$ la tasa de falla del componente $i$.

La función de confiabilidad del componente $i$ está dada por

$$
R_{i}(t)=e^{-\int_{0}^{t} \lambda_{i}(t) d t}=e^{-\lambda_{i} \int_{0}^{t} d t}=e^{-\lambda_{i} t}, \quad t \geq 0
$$

Al sustituir el valor de $R_{i}(t)$ en (4.3) se tiene la función de confiabilidad del sistema completo

$$
R_{s p}(t)=1-\prod_{i=1}^{n}\left(1-e^{-\lambda_{i} t}\right), \quad t \geq 0
$$

Ejemplo 4.4.2. Consideremos un sistema en paralelo formado por dos componentes, cuyas tasas de falla son $\lambda_{1} y \lambda_{2}$ respectivamente.

La confiabilidad del sistema es

$$
\begin{aligned}
R_{s p}(t) & =1-\left(1-e^{-\lambda_{1} t}\right)\left(1-e^{-\lambda_{2} t}\right) \\
& =e^{-\lambda_{1} t}+e^{-\lambda_{2} t}-e^{-\left(\lambda_{1}+\lambda_{2}\right) t}
\end{aligned}
$$

La función de densidad de falla es

$$
\begin{aligned}
f_{s p}(t) & =-\frac{d\left[e^{-\lambda_{1} t}+e^{-\lambda_{2} t}-e^{-\left(\lambda_{1}+\lambda_{2}\right) t}\right]}{d t} \\
& =\lambda_{1} e^{-\lambda_{1} t}+\lambda_{2} e^{-\lambda_{2} t}-\left(\lambda_{1}+\lambda_{2}\right) e^{-\left(\lambda_{1}+\lambda_{2}\right) t}
\end{aligned}
$$

Entonces la tasa de falla del sistema es

$$
h_{s p}(t)=\frac{\lambda_{1} e^{-\lambda_{1} t}+\lambda_{2} e^{-\lambda_{2} t}-e^{-\left(\lambda_{1}+\lambda_{2}\right) t}}{e^{-\lambda_{1} t}+e^{-\lambda_{2} t}-e^{-\left(\lambda_{1}+\lambda_{2}\right) t}}
$$

Observemos que ahora la tasa de falla $h_{s p}(t)$ es una función que depende de la edad del sistema, aunque la tasa de falla de cada componentes es constante. En la Figura 4.5 se muestra el comportamiento creciente de la tasa de falla del sistema con $\lambda_{1}=0.02$ y $\lambda_{2}=0.01$. 


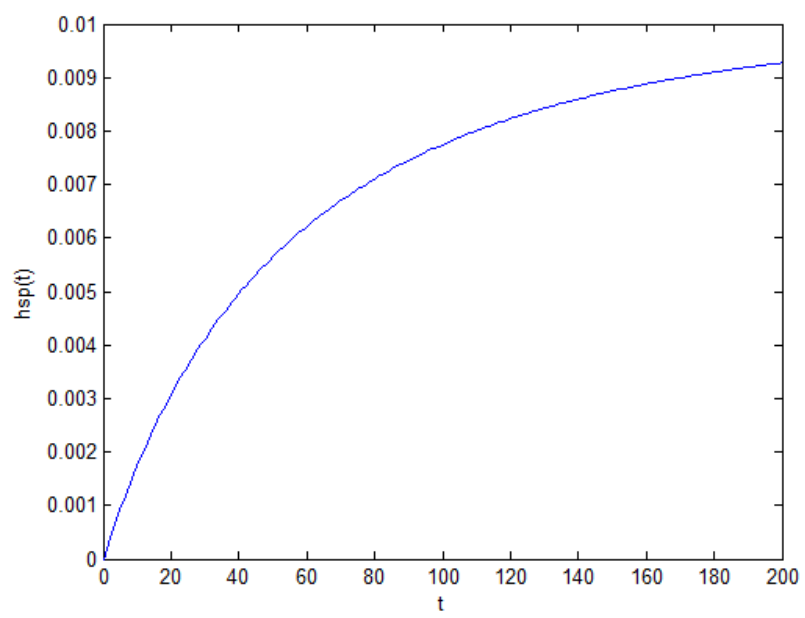

Figura 4.5: Tasa de falla de un sistema en paralelo con dos componentes con distribución exponencial.

¿Cómo se comporta el MTBF del sistema?

$$
\begin{aligned}
\operatorname{MTBF}_{s p} & =\int_{0}^{\infty} R_{s p}(t) d t \\
& =\int_{0}^{\infty} e^{-\lambda_{1} t}+e^{-\lambda_{2} t}-e^{-\left(\lambda_{1}+\lambda_{2}\right)} t d t \\
& =\frac{1}{\lambda_{1}} \frac{1}{\lambda_{2}}-\frac{1}{\lambda_{1}+\lambda_{2}}
\end{aligned}
$$

Ejemplo 4.4.3. Consideremos ahora un sistema con $n$ componentes i.i.d cuya distribución del tiempo de falla es exponencial. Sea $\lambda$ la tasa de falla de cada componente.

$$
R_{s p}(t)=1-\prod_{i=1}^{n}\left(1-e^{-\lambda t}\right)=1-\left(1-e^{-\lambda t}\right)^{n}
$$




$$
\begin{aligned}
\operatorname{MTBF}_{s p} & =\int_{0}^{\infty} R_{s p}(t) d t \\
& =\int_{0}^{\infty} 1-\left(1-e^{-\lambda t}\right)^{n} d t \\
& =\int_{0}^{\infty} 1-\sum_{j=0}^{n}\left(\begin{array}{l}
n \\
j
\end{array}\right) 1^{n-j}\left(-e^{-\lambda t}\right)^{j} d t \\
& =\int_{0}^{\infty} \sum_{j=1}^{n}\left(\begin{array}{c}
n \\
j
\end{array}\right)(-1)^{j+1} e^{-\lambda j t} d t \\
& =\sum_{j=1}^{n}\left(\begin{array}{l}
n \\
j
\end{array}\right)(-1)^{j+1} \int_{0}^{\infty} e^{-\lambda j t} d t \\
& =\left.\sum_{j=1}^{n}\left(\begin{array}{c}
n \\
j
\end{array}\right)(-1)^{j+1}\left(-\frac{1}{\lambda j}\right) e^{-\lambda j t}\right|_{0} ^{\infty} \\
& =\sum_{j=1}^{n}\left(\begin{array}{l}
n \\
j
\end{array}\right)(-1)^{j+1}\left(\frac{1}{\lambda j}\right) \\
& =\frac{1}{\lambda} \sum_{j=1}^{n}\left(\begin{array}{l}
n \\
j
\end{array}\right)(-1)^{j+1} \frac{1}{j} \\
& =\frac{1}{\lambda} \sum_{j=1}^{n} \frac{1}{j}
\end{aligned}
$$

\subsection{Política de reemplazo por edad para un sistema en paralelo}

Al igual que en el caso de los sistemas en serie, vamos a suponer para ambas políticas: reemplazo por edad e intervalos constantes, que los componentes del sistema en paralelo se reemplazan de manera conjunta.

La expresión del costo total esperado por unidad de tiempo para un sistema formado por $n$ componentes en paralelo, según la política de reemplazo por edad (3.5) está dada por 


$$
C_{s p}\left(t_{p}\right)=\frac{C_{p}\left[1-\prod_{i=1}^{n}\left(1-R_{i}\left(t_{p}\right)\right)\right]+C_{f} \prod_{i=1}^{n}\left(1-R_{i}\left(t_{p}\right)\right)}{\int_{0}^{t_{p}}\left[1-\prod_{i=1}^{n}\left(1-R_{i}(t)\right)\right] d t}, t_{p} \geq 0
$$

Donde $C_{p}$ y $C_{f}$ son los costos de mantenimiento preventivo y mantenimiento por falla respectivamente.

Ejemplo 4.5.1. Supongamos un sistema en paralelo formado por 2 componentes idénticos, para las cuales el tiempo de falla es modelado por la distribución Weibull con parámetro de escala $\lambda_{i}=0.01$ y parámetro de forma $\beta_{i}=3.5, i=1,2$. Cada componente puede ser reemplazado con un costo de $\$ 3,000.00$, por lo que el costo de reemplazar el sistema es de \$6,000.00; pero si un componente falla el costo de mantenimiento por falla es de $\$ 10,000.00$. ¿Qué tan frecuente debería ser reemplazado el sistema?

Vamos a calcular la función de confiabilidad del sistema.

$$
\begin{aligned}
R_{s p}(t) & =1-\left(1-R_{i}(t)\right)^{2} \\
& =1-\left(1-e^{-(0.01 t)^{3.5}}\right)^{2} \\
& =2 e^{-(0.01 t)^{3.5}}-e^{-2(0.01 t)^{3.5}}
\end{aligned}
$$

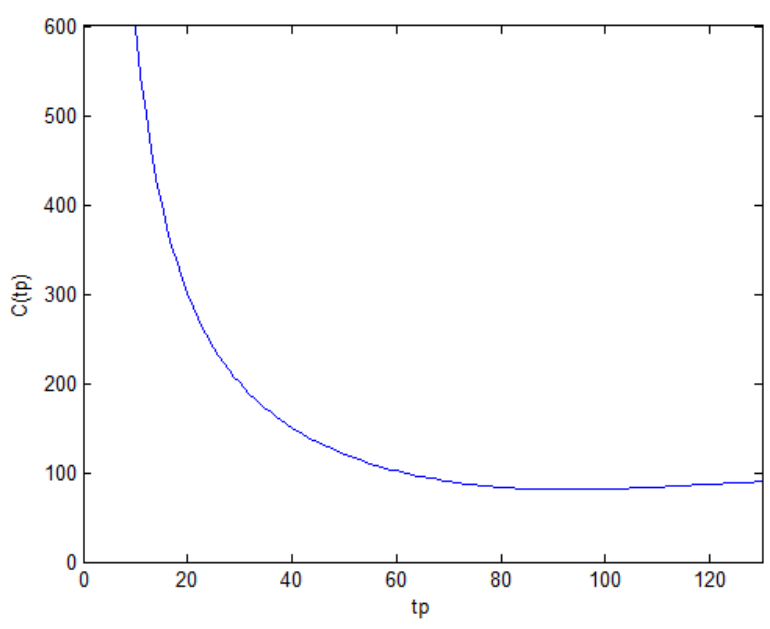

Figura 4.6: Costo esperado por unidad de tiempo para un sistema paralelo con dos componentes Weibull, según la política de reemplazo por edad.

Entonces para cada instante $t_{p} \geq 0$ 


$$
C_{s p}\left(t_{p}\right)=\frac{6000\left[2 e^{-(0.01 t)^{3.5}}-e^{-2(0.01 t)^{3.5}}\right]+10000\left[1-2 e^{-(0.01 t)^{3.5}}+e^{-2(0.01 t)^{3.5}}\right]}{\int_{0}^{t_{p}}\left[2 e^{-(0.01 t)^{3.5}}-e^{-2(0.01 t)^{3.5}}\right] d t}
$$

En la Figura 4.6 se muestra la curva del costo esperado por unidad de tiempo.

Por último, tenemos que el tiempo de reemplazo preventivo a costo mínimo, según la política de reemplazo por edad es $t_{p}=94$ unidades de tiempo con un costo mínimo de $\$ 81$ por unidad de tiempo.

\subsection{Política de reemplazo a intervalos constantes para un sistema en paralelo}

La expresión del costo total esperado por unidad de tiempo para un sistema formado por $n$ componentes en paralelo, según la política de reemplazo a intervalos constantes (3.8) está dada por

$$
C_{s p}\left(t_{p}\right)=\frac{C_{p}+C_{f} H_{s p}\left(t_{p}\right)}{t_{p}}, t_{p}>0
$$

$C_{p}$ y $C_{f}$ son los costos de mantenimiento preventivo y costo de mantenimiento por falla respectivamente y $H_{s p}$ el número esperado de fallas en el intervalo $\left(0, t_{p}\right)$.

Ejemplo 4.6.1. Supongamos un sistema en paralelo formado por 2 componentes idénticos, para las cuales el tiempo de falla es modelado por la distribución Weibull con parámetro de escala $\lambda_{i}=0.01$ y parámetro de forma $\beta_{i}=3.5, i=1,2$. Cada componente puede ser reemplazado con un costo de $\$ 3,000.00$, por lo que el costo de reemplazar el sistema es de $\$ 6,000.00$; pero si un componente falla el costo de mantenimiento por falla es de $\$ 10,000.00$. ¿Qué tan frecuente debería ser reemplazado el sistema según la política de reemplazo a intervalos constantes?

$$
\begin{aligned}
& \text { Primero vamos a calcular } H_{s p}\left(t_{p}\right) \text {. Sabemos que } h_{s p}(t)=\frac{f_{s p}(t)}{R_{s p}(t)} \text { con } \\
& \qquad \begin{aligned}
f_{s p}(t) & =-\frac{d\left[R_{s p}(t)\right]}{d t} \\
& =-\frac{d\left[1-\left(1-e^{-(0.01 t)^{3.5}}\right)^{2}\right]}{d t} \\
& =0.07(0.01 t)^{2.5} e^{-(0.01 t)^{3.5}}-0.07(0.01 t)^{2.5} e^{-2(0.01 t)^{3.5}}
\end{aligned}
\end{aligned}
$$


Asi que

$$
h_{s p}(t)=\frac{0.07(0.01 t)^{2.5} e^{-(0.01 t)^{3.5}}-0.07(0.01 t)^{2.5} e^{-2(0.01 t)^{3.5}}}{2 e^{-(0.01 t)^{3.5}}-e^{-2(0.01 t)^{3.5}}}
$$

En este caso

$$
C_{s p}\left(t_{p}\right)=\frac{6000+10000 \int_{0}^{t_{p}} h_{s p}(t) d t}{t_{p}}, t_{p}>0
$$

En la Figura 4.7 se muestra la curva del costo esperado por unidad de tiempo.

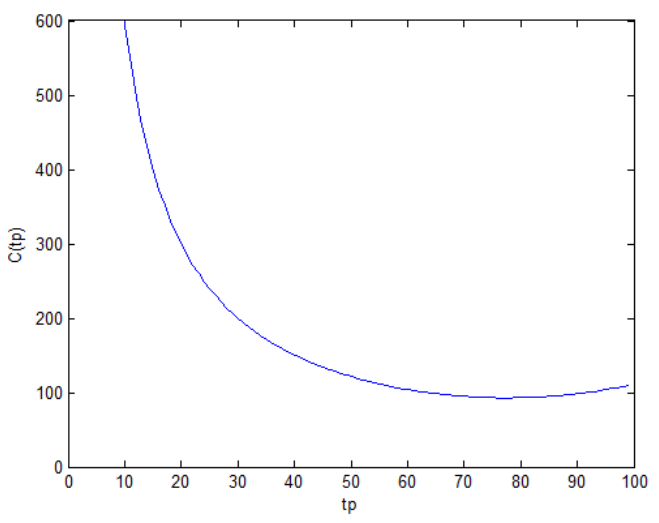

Figura 4.7: Costo esperado por unidad de tiempo para un sistema paralelo con dos componentes Weibull.

Por último, tenemos que el tiempo de reemplazo preventivo a costo mínimo, según la política de reemplazo a intervalos constantes es $t_{p}=78$ unidades de tiempo con un costo mínimo de $\$ 93$ por unidad de tiempo.

Al igual que en el caso de los sistemas en serie, para los sistemas en paralelo, la política de reemplazo por edad sugiere un óptimo con tiempos de reemplazo mayores que la política de reemplazo a intervalos constantes, y por ende, el costo es menor. También hay que observar que en el caso de los sistemas en serie, los intervalos de reemplazo óptimo, para ambas políticas, son menores que los intervalos de reemplazo óptimos para los sistemas en paralelo. Esto se debe a que las configuraciones en paralelo aumentan su confiabilidad al incrementar el número de componentes. 


\subsection{Sistemas Mixtos}

Consideremos ahora el siguiente sistema, en el cual se tiene una combinación de sistemas en serie y en paralelo.

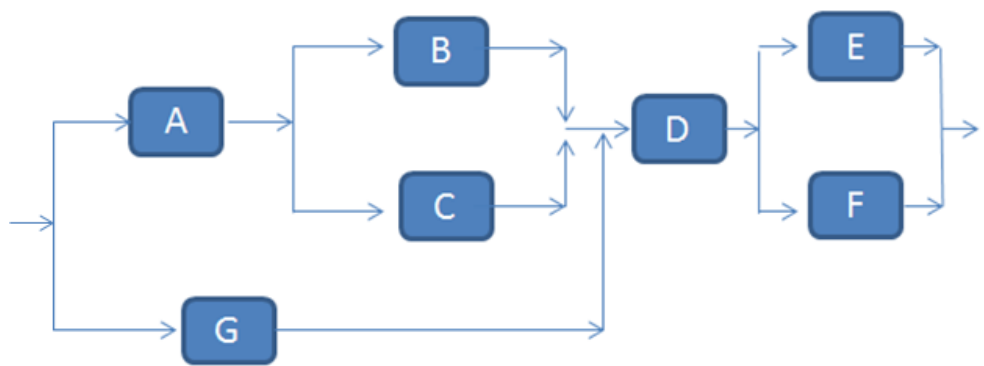

Figura 4.8: Sistema formado por componentes en serie y en paralelo
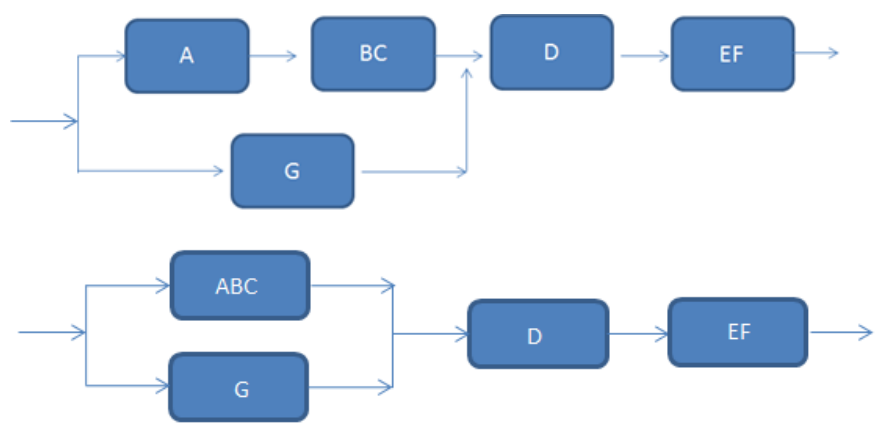

$$
A B C G
$$

D

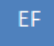

Figura 4.9: Simplificación del sistema mixto

Para poder obtener la confiabilidad de un sistema mixto hay que ir resolviendo por etapas, calculando la confiabilidad de cada uno de los subsistemas en serie o en paralelo formados en cada etapa.

Para calcular la confiabilidad del sistema mostrado en la Figura 4.8 lo primero que podríamos hacer es encontrar la confiabilidad de los dos pares de componentes colocados en paralelo (cada uno de estos pares se ve como un subsistema). Después habría que resolver la confiabilidad del subsistema en serie, cuyos componentes son A y BC. Enseguida se calcula la confiabilidad del subsistema en paralelo con componentes ABC y G. Por último, se calcula la confiabilidad del sistema en serie formado 
por los componentes ABCG, D y EF. En la Figura 4.9 se muestra la representación en diagramas de bloques que habría que ir resolviendo.

Para ilustrar las políticas de reemplazo por edad y reemplazo a intervalos constantes en un sistema mixto se consideran tres componentes A, B y C cuyos tiempos de falla son modelados por la distribución Weibull. Estos tres componentes se organizan en distintas configuraciones: sistema en serie, sistema en paralelo y sistema mixto. Para cada política de reemplazo vamos a realizar un análisis de los intervalos de reemplazo óptimo para cada uno de los componentes por sí solos, y para cada una de las tres configuraciones, en las cuales suponemos que los tres componentes se reemplazan de manera conjunta. En el Cuadro 4.1 se muestran los parámetros de la distribución junto con los valores de $C_{p}$ y $C_{f}$ para cada componente.

Cuadro 4.1: Características de los componentes

\begin{tabular}{|c|c|c|c|c|}
\hline Componente & Parámetro de forma & Parámetro de escala & $C_{p}$ & $C_{f}$ \\
\hline A & 2 & 0.001 & $\$ 5,000$ & $\$ 39,000$ \\
B & 3 & 0.005 & $\$ 7,000$ & $\$ 44,000$ \\
C & 2.5 & 0.008 & $\$ 21,000$ & $\$ 48,000$ \\
\hline
\end{tabular}

Primero vamos a buscar el intervalo de reemplazo óptimo señalado por cada política, para cada uno de los componentes.

Cuadro 4.2: Reemplazo por edad

\begin{tabular}{|c|c|c|}
\hline Componente & Intervalo de reemplazo óptimo & Costo mínimo $(\$ /$ día $)$ \\
\hline A & 388 & 26.40 \\
B & 91 & 116.13 \\
C & 99 & 380.81 \\
\hline
\end{tabular}

Cuadro 4.3: Reemplazo a intervalos constantes

\begin{tabular}{|c|c|c|}
\hline Componente & Intervalo de reemplazo óptimo & Costo mínimo(\$/día $)$ \\
\hline A & 358 & 27.93 \\
B & 86 & 122.07 \\
C & 76 & 458.36 \\
\hline
\end{tabular}




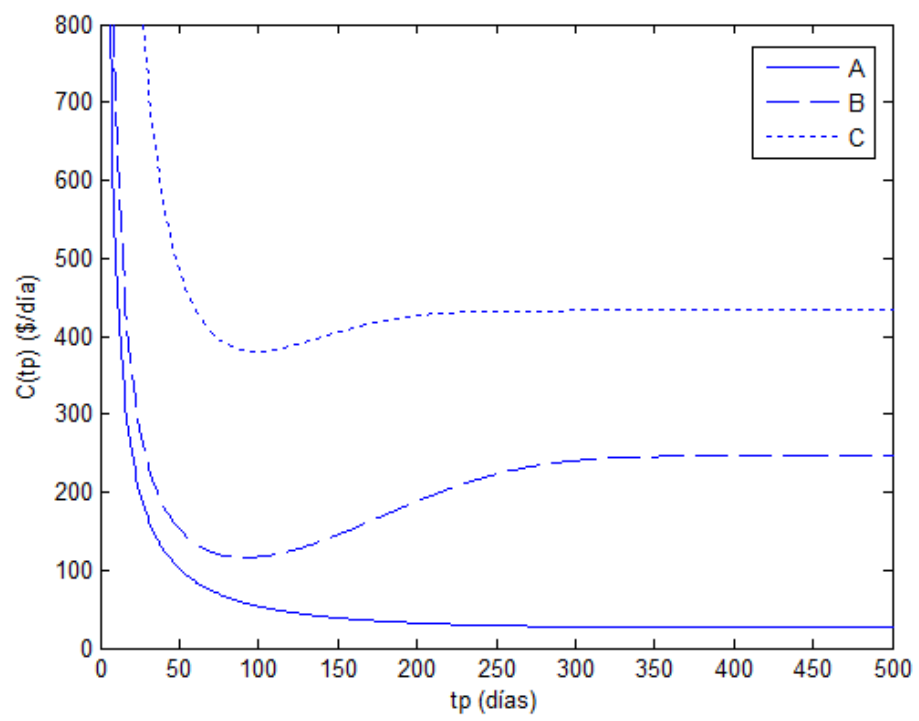

Figura 4.10: Costo esperado por unidad de tiempo/Reemplazo por edad

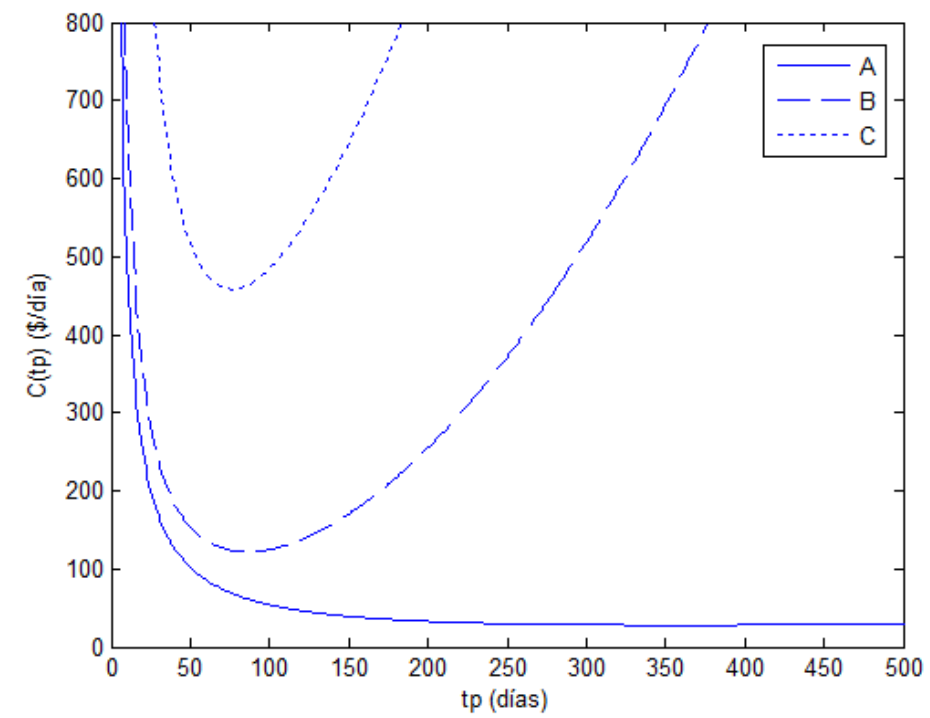

Figura 4.11: Costo esperado por unidad de tiempo/Reemplazo a intervalos constantes

Consideremos ahora la configuración mixta mostrada en la Figura 4.12. Vamos a obtener el intervalo de reemplazo óptimo en ambas políticas, suponiendo que los tres componentes se reemplazan de manera conjunta, es decir, considerando el sistema como un solo componente. 


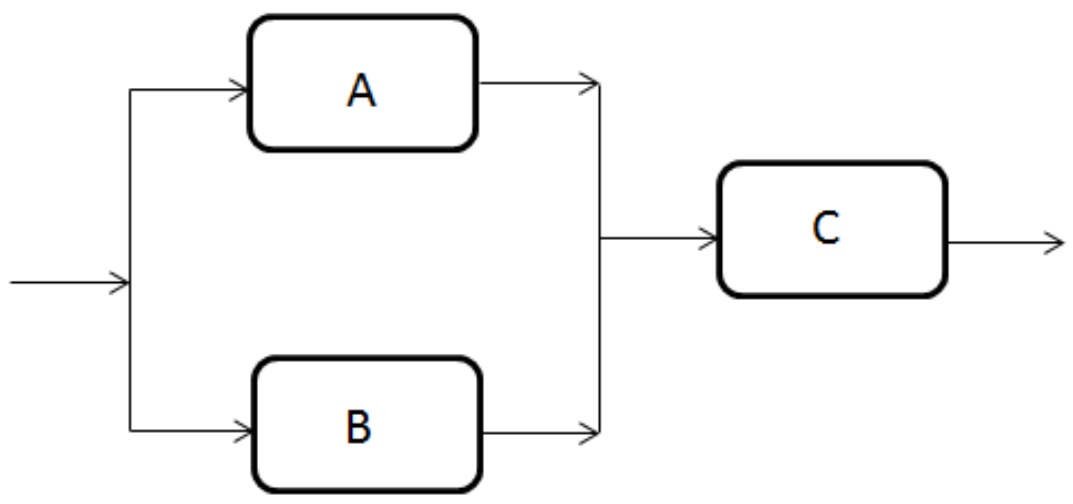

Figura 4.12: Sistema mixto

Cuadro 4.4: Reemplazo de componentes del sistema mixto

\begin{tabular}{|c|c|c|}
\hline $\begin{array}{c}\text { Política } \\
\text { de reemplazo }\end{array}$ & $\begin{array}{c}\text { Intervalo de reemplazo óptimo } \\
\text { (días) }\end{array}$ & $\begin{array}{c}\text { Costo mínimo } \\
(\$ / \text { día })\end{array}$ \\
\hline $\begin{array}{c}\text { Edad } \\
\text { Intervalos constantes }\end{array}$ & 113 & 387.00 \\
105 & 410.76 \\
\hline
\end{tabular}

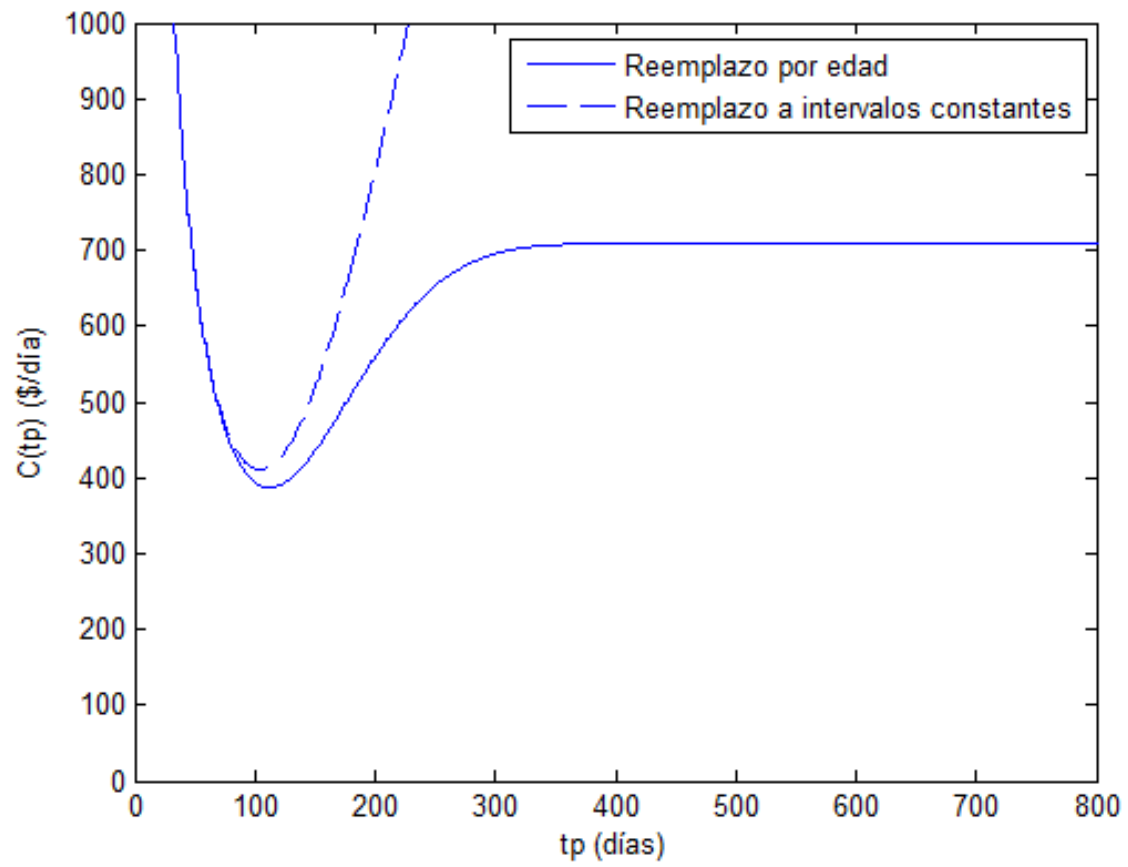

Figura 4.13: Costo esperado por unidad de tiempo/Reemplazo conjunto 


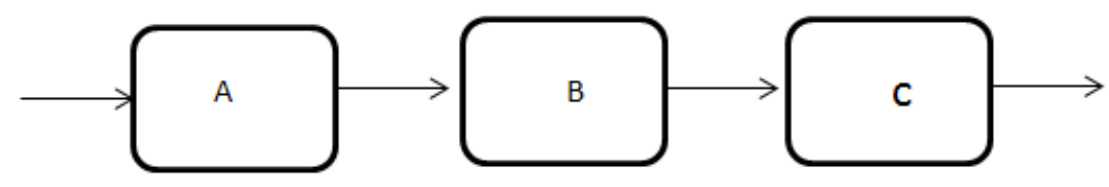

Figura 4.14: Sistema en serie

Ahora vamos a obtener el intervalo de reemplazo óptimo del sistema, visto como uno solo, considerando que los tres componentes están colocados en serie como se muestra en la Figura 4.14.

Cuadro 4.5: Reemplazo conjunto de componentes del sistema en serie

\begin{tabular}{|c|c|c|}
\hline $\begin{array}{c}\text { Política } \\
\text { de reemplazo }\end{array}$ & $\begin{array}{c}\text { Intervalo de reemplazo óptimo } \\
\text { (días) }\end{array}$ & $\begin{array}{c}\text { Costo mínimo } \\
(\$ / \text { día })\end{array}$ \\
\hline Edad & 65 & 875.32 \\
Intervalos constantes & 57 & 962.23 \\
\hline
\end{tabular}

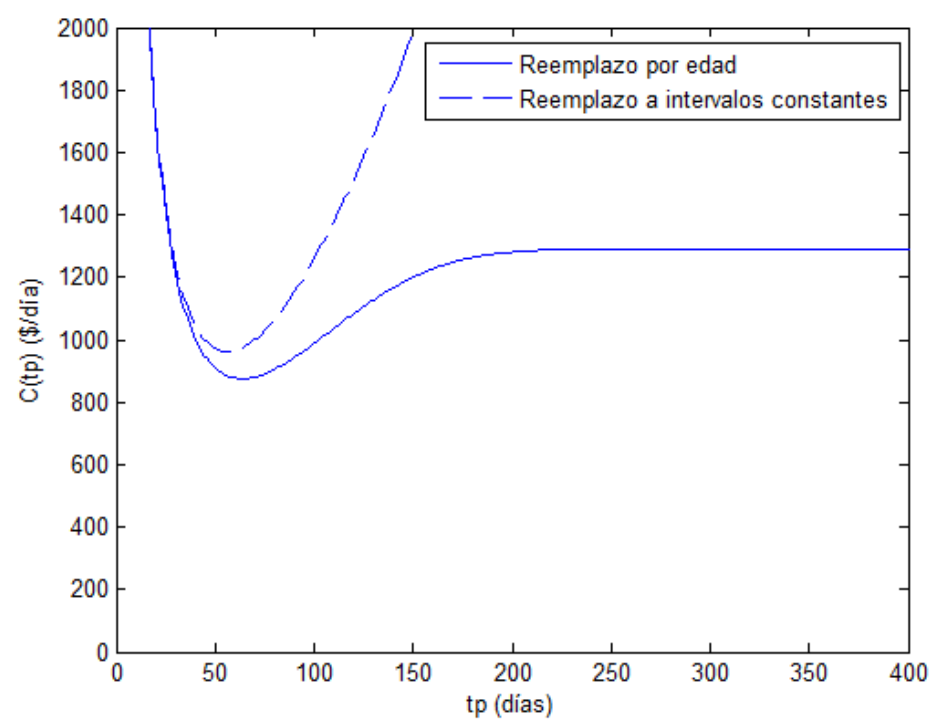

Figura 4.15: Costo esperado por unidad de tiempo/Sistema en serie

Por último, considerando que los tres componentes están colocados en paralelo como se muestra en la Figura 4.16, obtenemos el intervalo de reemplazo óptimo del sistema visto como uno solo. 


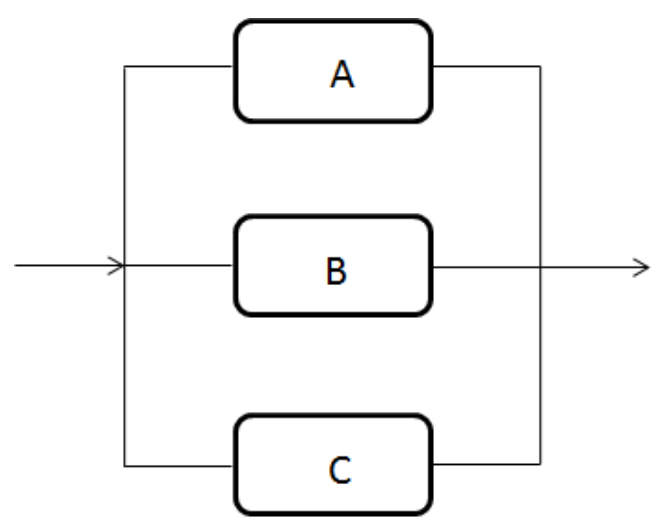

Figura 4.16: Sistema en paralelo

Cuadro 4.6: Reemplazo conjunto de componentes del sistema en paralelo

\begin{tabular}{|c|c|c|}
\hline $\begin{array}{c}\text { Política } \\
\text { de reemplazo }\end{array}$ & $\begin{array}{c}\text { Intervalo de reemplazo óptimo } \\
(\text { días })\end{array}$ & $\begin{array}{c}\text { Costo mínimo } \\
(\$ / \text { día })\end{array}$ \\
\hline Edad & 595 & 116.41 \\
Intervalos constantes & 501 & 131.09 \\
\hline
\end{tabular}

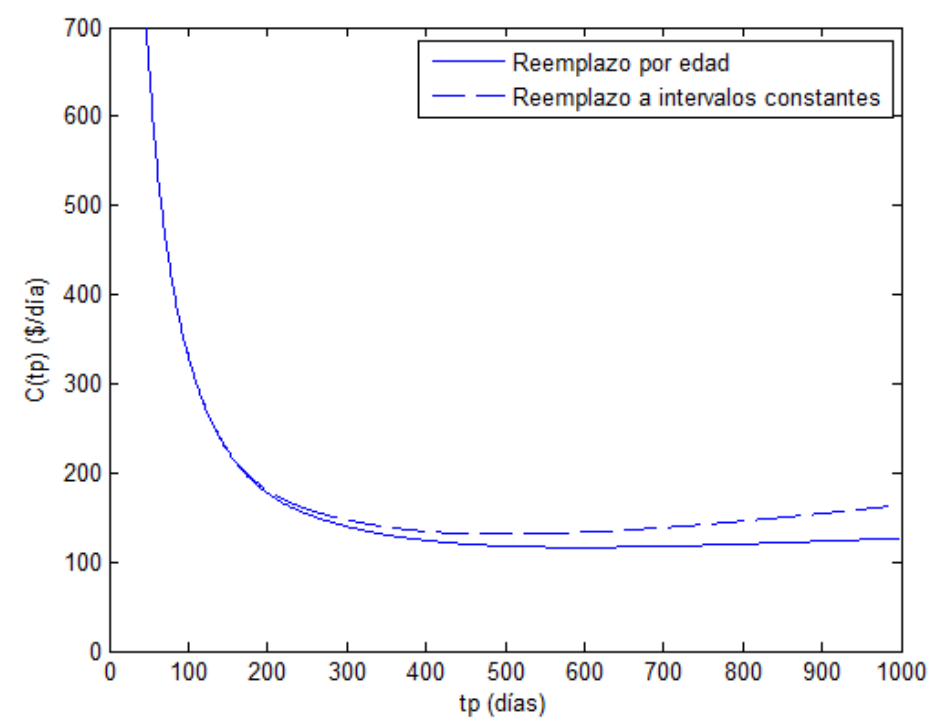

Figura 4.17: Costo esperado por unidad de tiempo/Sistema en paralelo

De los resultados obtenidos para cada una de las tres configuraciones, se puede observar que la configuración en paralelo resulta ser la mejor estrategia, comparada con las configuraciones mixta y en serie, pues en estas el costo es mayor. De estas dos 
últimas, la configuración es serie es la que señala el intervalo de reemplazo óptimo más pequeño, 65 días en el caso de reemplazo por edad y 57 días en el caso de reemplazo a intervalos constantes. Cabe mencionar que con estos enfoques se podría tener un ahorro en los costos de mantenimiento si en lugar de reemplazar los tres componentes, sólo se reemplaza el componente que falló.

\subsection{Mantenimiento Oportunista para Sistemas en Serie}

Los modelos de mantenimiento oportunista, básicamente se refieren a situaciones en las que el mantenimiento preventivo se realiza tomando oportunidades. Una oportunidad puede ser una falla del sistema, inspecciones de calidad y otras situaciones donde el sistema deja de funcionar.

Para la mayoría de los sistemas que operan de manera continua, como por ejemplo, generadores de energía, petroquímicas e instalaciones petrolíferas en alta mar, el costo de una parada de producción puede ser muy costoso, por lo tanto se busca limitar el número de paradas mediante la combinación de varias tareas de mantenimiento con la finalidad de reducir los costos totales de mantenimiento.

El objetivo de la política de mantenimiento oportunista es ahorrar costos cuando conviene realizar el reemplazo de varios componentes en lugar de reemplazarlos por separado.

\subsubsection{Descripción del Sistema}

Considérese un sistema compuesto de $q$ componentes colocados en serie. En éste, la falla de cualquier componente ocasiona la falla del sistema. El objetivo de la política es seleccionar la combinación de los tiempos de reemplazo de los componentes del sistema, junto con el intervalo periódico de reemplazo $\tau$ que generen el costo esperado por unidad de tiempo mínimo.

Sean $\tau_{1}, \tau_{2}, \ldots, \tau_{q}$ los intervalos de reemplazo preventivo óptimos correspondientes a los componentes $1,2, \ldots, q$ respectivamente. Se supone que en cada reemplazo, ya 
sea preventivo o correctivo, el componente queda como nuevo. Durante cada parada del sistema por mantenimiento correctivo, se debe decidir si se puede tomar la oportunidad de reemplazar de manera preventiva algunos de los componentes que no fallaron. La decisión estará basada en la degradación del componente y el riesgo que se toma si estos componentes fallan antes de alcanzar el siguiente reemplazo preventivo programado.

\subsubsection{Estructura de costos}

En esta sección vamos a clasificar los costos de las actividades de mantenimiento en dos categorías: costos fijos y costos variables.

Las actividades que generan los costos fijos del mantenimiento son: la movilización de un equipo de reparación, herramientas, transporte y desmontaje y montaje del sistema. El costo fijo de mantenimiento es generado independientemente del número de componentes que sean reemplazados en el sistema. Cuando se combinan varias acciones de mantenimiento, el costo fijo se comparte entre éstas y de esta forma se reducen los costos totales de mantenimiento.

Sean $C_{o}^{p}$ y $C_{o}^{c}$ los costos fijos de mantenimiento preventivo y correctivo respectivamente. Consideraremos que $C_{o}^{p}<C_{o}^{c}$.

Los costos variables están relacionados con las características específicas del componente que será reemplazado tales como: repuestos, mano de obra, herramientas específicas y procedimientos de reparación. También se incluye la pérdida de producción generada durante el tiempo en que se realizan las actividades de mantenimiento. Sean $C_{i}^{p}$ el costo específico de mantenimiento preventivo para el componente $i$ y $C_{i}^{c}$ el costo de mantenimiento correctivo para el componente $i$. Nuevamente $C_{i}^{p}<C_{i}^{c}$.

Cuando se lleva a cabo un mantenimiento preventivo, el costo total en el sistema es

$$
C_{s}^{p}=C_{o}^{p}+\sum_{i \in G p} C_{i}^{p}
$$

$G p$ es el grupo de componentes que serán reemplazados de manera preventiva.

Cuando se efectúa un mantenimiento correctivo, por la falla del j-ésimo componente, se tiene la oportunidad de reemplazar otros componentes críticos, de acuerdo a una regla definida. El costo correctivo del sistema por la falla del componente $j$, $C_{s, j}^{c}$ está dado por 


$$
C_{s, j}^{c}=C_{o}^{c}+C_{j}^{c}+\sum_{i \in G_{h}, i \neq j} C_{i}^{p}
$$

$G_{h}$ es el grupo de componentes que serán reemplazados preventivamente durante esta oportunidad. El grupo $G_{h}$ se definirá con base al deterioro del componente y el riesgo tomado si este componente falla antes de alcanzar el siguiente reemplazo.

Vamos a considerar el caso en el que el j-ésimo componente falla en el tiempo $t_{j}$, entre dos reemplazos programados $k \tau$ y $(k+1) \tau$. La oportunidad de reemplazar el componente $i$ genera un costo esperado $C_{i}^{p} R_{i}\left(t_{j}\right)$. Si el componente $i$ no se reemplaza en ese momento, puede ocurrir una de las siguientes dos posibilidades:

- El componente $i$ continúa operando hasta el siguiente reemplazo programado, lo cual originará un costo $C_{i}^{p} R_{i}\left((k+1) \tau \mid t_{j}\right)$.

- El componente $i$ falla en el tiempo $t$ antes del siguiente reemplazo programado, lo cual originará un costo $\left(C_{o}^{c}+C_{i}^{c}\right) F_{i}(t)$

La decisión de llevar a cabo o no un reemplazo oportunista del componente $i$, dependerá de la comparación de los costos. Si el costo de efectuar el reemplazo oportunista es menor que el costo correctivo, es mejor cambiar el componente, en caso contrario, el componente se cambiará hasta su siguiente reemplazo programado. Es decir, si

$$
\left.C_{i}^{p}\left(R_{i}\left(t_{j}\right)-R_{i}\left((k+1) \tau \mid t_{j}\right)\right)<\left(C_{o}^{c}+C_{i}^{c}\right) F_{i}\left((k+1) \tau \mid t_{j}\right)\right)
$$

Se realiza el reemplazo oportunista del componente $i$ en el tiempo $t_{j}$. En caso contrario, se deja hasta el siguiente reemplazo programado.

\subsubsection{Descripción del modelo}

El tiempo de reemplazo base $\tau$ se define como el tiempo de reemplazo mínimo de todos los componentes, es decir, $\tau=\min _{i=1, \ldots, q} \tau_{i}$, donde $\tau_{i}$ es el tiempo de reemplazo óptimo del componente $i$, según la política de reemplazo basado en la edad. Los nuevos intervalos de reemplazo de los componentes se definen como múltiplos del intervalo de mantenimiento $\tau$, es decir, $\nu_{i}=k_{i} \tau$, donde $k_{i}$ son enteros que satisfacen $k_{i} \geq 1, \quad i=1,2, \ldots, q$. Para cada componente, el valor inicial $k_{i}$ es calculado

dividiendo el intervalo de reemplazo óptimo del componente $i\left(\tau_{i}\right)$ por el intervalo óptimo de reemplazo mínimo en el sistema, redondeando al entero más cercano. 


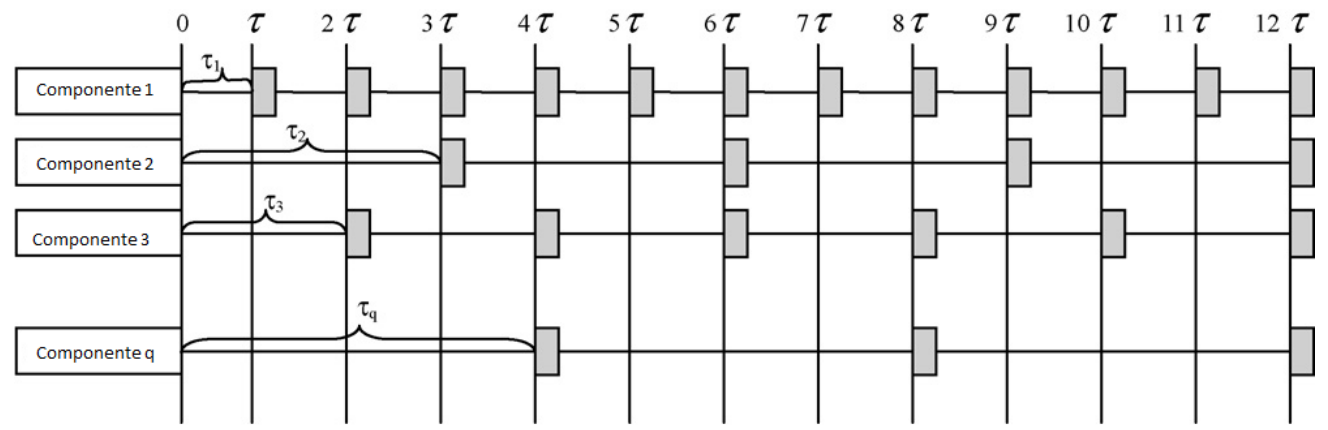

Figura 4.18: Plan de mantenimiento preventivo programado, sin considerar fallas (Laggoune et al.,2009, pág. 1501)

De lo anterior se tiene que las variables de decisión del modelo son $\left(\tau, k_{1}, k_{2}, \ldots, k_{q}\right)$, donde $\tau \geq 0$ es variable continua y las variables $k_{i}$ son enteras.

En la expresión del costo total esperado por unidad de tiempo intervienen los costos generados a lo largo del ciclo de vida del sistema. Un ciclo va a estar dado por el lapso de tiempo durante el cual todos los componentes del sistema se reemplazan de manera simultánea, y se denotará por $K \tau$, con $K=\operatorname{mcm}\left\{k_{1}, k_{2}, \ldots, k_{q}\right\}$. En la Figura 4.18 se muestra un sistema, el cual es renovado de manera completa en $12 \tau$. En este punto todos los componentes del sistema son reemplazados de manera simultánea.

El costo total esperado por unidad de tiempo de expresa como sigue:

$$
\mathrm{C}\left(\tau, k_{1}, ., k_{q}\right)=\frac{\sum_{k=1}^{K} \sum_{j=1}^{q}\left[\left(C_{o}^{c}+C_{j}^{c}+\sum_{i \in G_{h k}, i \neq j} C_{i}^{p}\right) F_{s j}(k \tau)+\left(C_{o}^{p}+\sum_{i \in G_{p k}} C_{i}^{p}\right) R_{s j}(k \tau)\right]}{K \tau}
$$

donde $F_{s j}(\cdot)$ es la función de distribución acumulada del sistema, debido a la falla del j-ésimo componente. Recordemos que para un componente en serie la falla de cualquier componente origina la falla del sistema, entonces $F_{s j}(\cdot)=F_{j}(\cdot)$. El grupo $G_{h k}$, es el grupo de componentes que se reemplazaran de manera oportunista cuando una falla ocurre en el intervalo $((k-1) \tau, k \tau)$ y $G_{p k}$ es el grupo de componentes que serán reemplazados de manera preventiva en el instante $k \tau$.

En la Figura 4.19 se presenta el diagrama de flujo del algoritmo que se utiliza para encontrar el conjunto óptimo $\left(\tau, \mathrm{k}_{1}, k_{2}, \ldots, k_{q}\right)$, donde $\tau$ indica el tiempo mínimo de reemplazo y cada $k_{i}, i=1, \ldots, q$ fija el tiempo de reemplazo de cada componente, el cual se obtiene al multiplicar $k_{i} \tau$. Como la función que describe el costo esperado por unidad de tiempo está en función de variables continuas y discretas, el algoritmo se basa en simulaciones 
Monte Carlo, el cual puede manejar ambas variables. Se generan muestras aleatorias de los tiempos de vida de cada uno de los componentes y son usadas para simular el costo total de mantenimiento para diferentes combinaciones $\left(\tau, k_{1}, k_{2}, \ldots, k_{q}\right)$.

Como el algoritmo de búsqueda requiere un punto inicial, la solución inicial puede ser definida por los tiempos de reemplazo óptimos de cada componente. Estos tiempos proporcionan los valores iniciales $k_{i}$. Para el número de combinaciones de los valores de $k_{i}$, $i=1, \ldots q$ en lugar de usar el factorial, se reducirán éstas suponiendo que el reemplazo de cada componente sólo puede ser adelantado o retrasado un periodo $\tau$ de su valor original, es decir, $k_{i}-1 \leq k_{i} \leq k_{i}+1, i=1, \ldots, q$.

\section{Algoritmo}

1. Generar muestras aleatorias de los tiempos de vida de los componentes, $t_{i}$, de acuerdo a la distribución de probabilidad de falla. El tiempo de falla del sistema es definido por $t_{s}=\min _{i=1, \ldots, q} t_{i}$. Se identifica el componente que produce la falla del sistema. Los reemplazos son programados en los tiempos $k \tau$, donde $k$ es un entero que varía de 0 a $K$.

2. En el k-ésimo reemplazo, el tiempo de falla $t_{s}$ es comparado con el tiempo programado para el reemplazo preventivo $k \tau$. Dos posibilidades existen:

i) Si no existe una falla antes de $k \tau$, se lleva a cabo el mantenimiento preventivo programado en $k \tau$, de acuerdo al plan actual. Se pasa al siguiente reemplazo programado $(k+1) \tau$.

ii) Si una falla es observada, falla el sistema y se reemplaza de manera correctiva el componente que falló. En base a la regla descrita arriba, se decide si se toma la oportunidad de reemplazar los otros componentes. Se calculan los costos preventivos.

3. Se generan nuevos tiempos de vida para los componentes reemplazados en el paso 2 (pues nuevos componentes son instalados). Se pasa al siguiente reemplazo programado $(k+1) \tau$ y así hasta que se alcanza el tiempo total para reemplazar todos los componentes de manera simultánea.

4. Repetir los pasos 1-3 para generar nuevos escenarios de manera aleatoria, hasta que se alcanza un número de simulaciones $N$.

5. El costo total esperado por unidad de tiempo está dado por

$$
\frac{1}{N} \sum_{s=1}^{N}\left[\sum_{k=1}^{K}\left(\left(C_{o}^{c}+C_{j}^{c}+\sum_{i \in G_{h}, i \neq j} C_{i}^{p}\right) I_{F_{k, s}}+\left(C_{o}^{p}+\sum_{i \in G p} C_{i}^{p}\right) I_{R_{k, s}}\right)\right]
$$

Donde $N$ es el número de simulaciones, $I_{F_{k, s}}$ y $I_{R_{k, s}}$ son funciones indicadoras para los 
estados de falla y operación respectivamente. Para el k-ésimo intervalo de reemplazo de simulación $s$, estas funciones se definen como:

$$
\begin{gathered}
I_{F_{k, s}}=\left\{\begin{array}{lll}
1, & \text { si el sistema falla } \\
0, & \text { si el sistema opera. }
\end{array}\right. \\
I_{R_{k, s}}=\left\{\begin{array}{lll}
1, & \text { si el sistema opera } \\
0, & \text { si el } & \text { sistema falla. }
\end{array}\right.
\end{gathered}
$$

6. El procedimiento es repetido para diferentes combinaciones de los valores $k_{i}$.

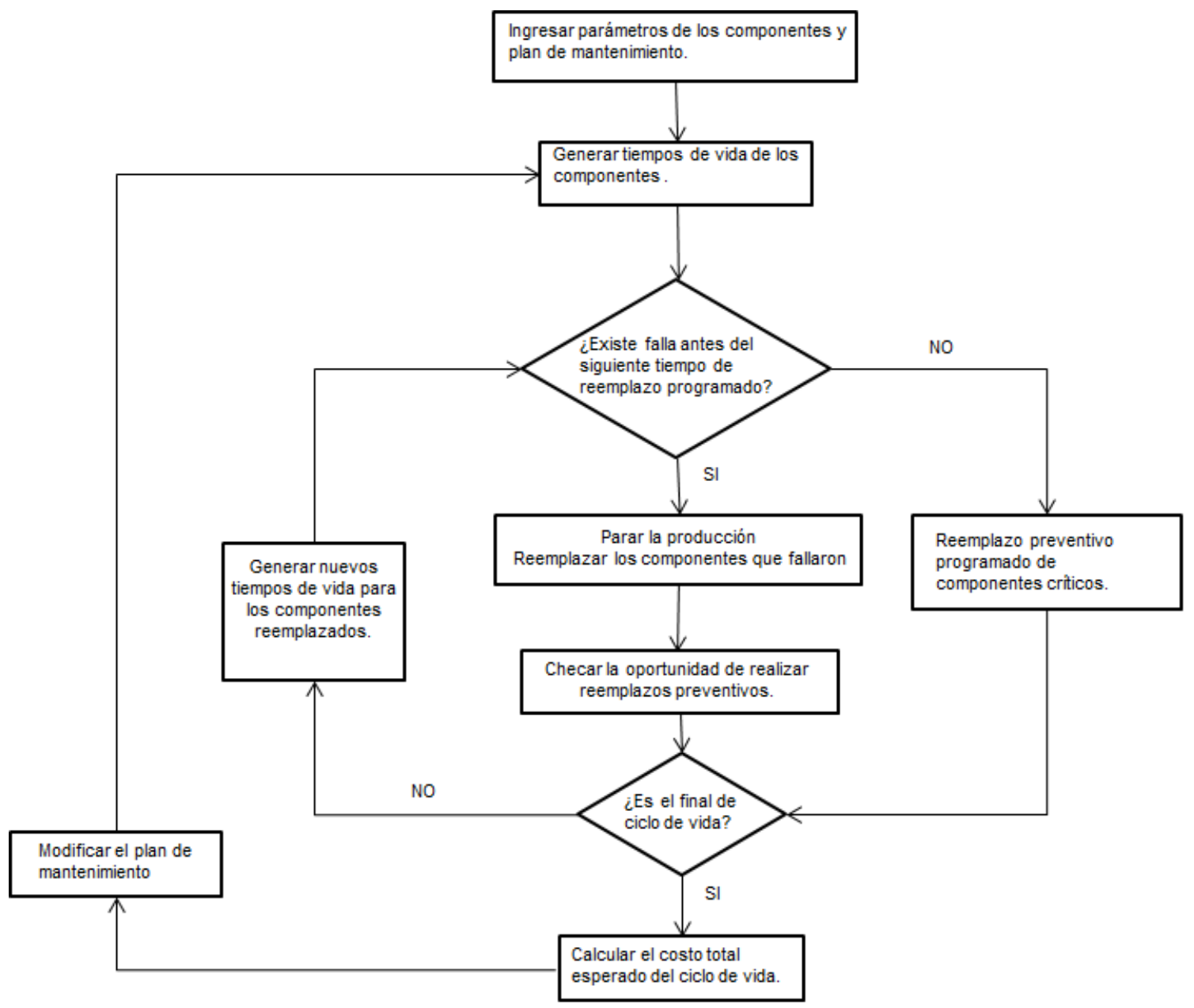

Figura 4.19: Diagrama de flujo para el algoritmo que permite encontrar el intervalo de reemplazo óptimo y la estrategia de grupo (Laggoune et al.,2009, pág. 1501) 
En el siguiente ejemplo se ilustra el procedimiento de mantenimiento oportunista para un sistema en serie formado por dos componentes. Se comparan los resultados obtenidos con los que se obtienen con las políticas de mantenimiento basado en la edad y mantenimiento a intervalos constantes.

Ejemplo 4.8.1. Vamos a considerar un sistema en serie formado por dos componentes A y $B$, con distintas tasas de falla. Calcularemos los tiempos de reemplazo óptimos de los componentes usando los distintos enfoques que se han estudiado hasta el momento: reemplazo basado en la edad, reemplazo conjunto, es decir, considerando el sistema como un solo componente y reemplazo oportunista. Al terminar compararemos los resultados obtenidos.

Se supondrá que los costos fijos tanto de mantenimiento preventivo como correctivo son $\$ 600.00$ y $\$ 8,0000.00$ respectivamente. En el Cuadro 4.7 se muestran los datos correspondientes a los parámetros de la distribución de los tiempos de falla de cada componente, junto con los costos variables de mantenimiento preventivo y correctivo.

Cuadro 4.7: Características de los componentes

\begin{tabular}{|c|c|c|c|c|}
\hline Componente & Parámetro de forma & Parámetro de escala & $C_{i}^{p}$ & $C_{i}^{c}$ \\
\hline A & 2.5 & 500 & $\$ 600$ & $\$ 5,000.00$ \\
B & 2.7 & 200 & $\$ 200$ & $\$ 400.00$ \\
\hline
\end{tabular}

Para empezar vamos a calcular el intervalo de mantenimiento preventivo óptimo de cada componente (sin considerar la configuración en serie), de acuerdo a la política de reemplazo preventivo basada en la edad. En el Cuadro 4.8 se muestran los óptimos para cada componente.

Cuadro 4.8: Reemplazo Basado en la Edad

\begin{tabular}{|c|c|c|}
\hline Componente & $t_{\text {opt }}$ & Costo mínimo $(\$ /$ día $)$ \\
\hline $\mathrm{A}$ & 171 & 11.81 \\
$\mathrm{~B}$ & 72 & 17.90 \\
\hline
\end{tabular}

En la Figura 4.20 se muestra el comportamiento del costo esperado por unidad de tiempo para cada cada componente.

Ahora veamos qué sucede con el reemplazo conjunto de los componentes considerando la configuración en serie. En este caso el tiempo de reemplazo óptimo para los dos componentes del sistema es 73 días, con un costo minimo de $\$ 31.51$ por día. En la Figura 4.21 se muestra el comportamiento del costo esperado por unidad de tiempo para el sistema. 


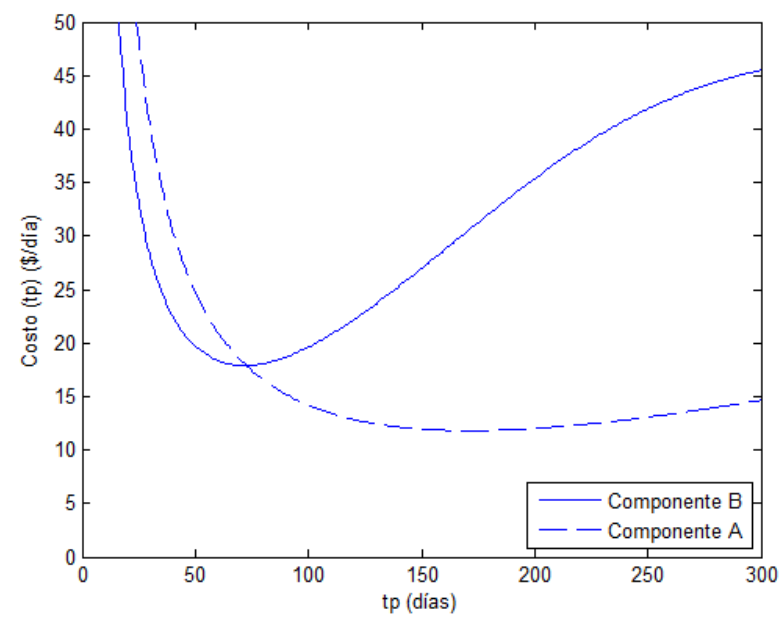

Figura 4.20: Reemplazos separados

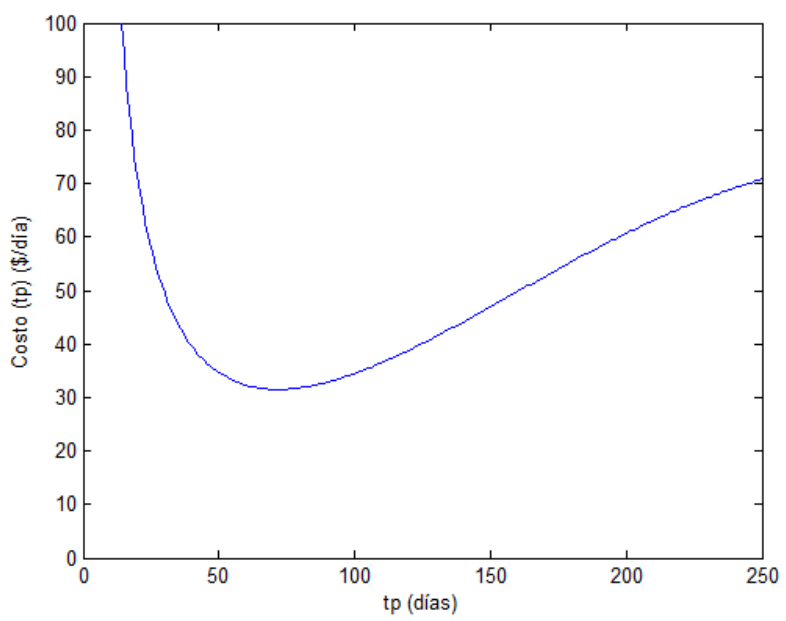

Figura 4.21: Reemplazo conjunto

Por último, vamos a utilizar el enfoque de mantenimiento oportunista para obtener la combinación de los tiempos de reemplazo para cada componente que minimize los costos totales de mantenimiento preventivo. Llamaremos componente 1 al componente A y componente 2 al componente $B$.

Según el algoritmo que se describió anteriormente, el valor de $\tau$ es 72 días. Los valores iniciales para los tiempos de reemplazo individuales son $k_{1}=2$ y $k_{2}=1$, es decir, el componente 1 se reemplaza cada dos reemplazos del componente 2 (cada $2 \tau$ ). Se realizan 50,000 simulaciones para generar los distintos escenarios y obtener el resultado óptimo. En el Cuadro 4.9 se muestran los resultados obtenidos para las seis diferentes combinaciones 
que se obtuvieron a partir del grupo inicial $\{2,1\}$.

Cuadro 4.9: Solución para diferentes grupos de reemplazo

\begin{tabular}{|c|c|c|c|}
\hline Combinación $k_{1}, k_{2}$ & Ciclo de vida del sistema(días) & $\tau($ días $)$ & Costo $(\$ /$ día) \\
\hline 1,1 & 71 & 71 & 27.1246 \\
2,1 & 140 & 70 & 24.5876 \\
2,2 & 134 & 67 & 31.1893 \\
1,2 & 136 & 68 & 26.2368 \\
3,1 & 198 & 66 & 26.4678 \\
3,2 & 384 & 64 & 28.2886 \\
\hline
\end{tabular}

Se puede observar que de las 6 combinaciones, la $\{2,1\}$ es la que minimiza los costos totales de mantenimiento. De hecho, de los tres enfoques este último es el que origina los menores costos. En el reemplazo individual de los componentes, reemplazar los dos componentes genera un costo de $\$ 29.71$, y si éstos se reemplazan de manera conjunta se genera un costo de $\$ 31.51$.

Los datos de este problema fueron tomados de [10] . Los resultados que se han obtenido no coinciden con los resultados que presenta el artículo, pues en éste se usa información no publicada.

\subsection{Conclusiones}

En esta tesis se ha realizado un análisis estadístico de los tiempos de falla correspondientes a un componente o sistema. Se estudiaron distintos procedimientos para estimar ya sea la función de distribución del tiempo de falla $F(\cdot)$ o la función de confiabilidad $R(\cdot)$, tanto para datos completos como para datos censurados. A partir de estas funciones se pueden derivar otros de los parámetros que aportan información sobre las confiabilidad de un componente o sistema como son: la tasa de falla y el $M T B F$.

Se estudiaron las distintas políticas de mantenimiento o reemplazo preventivo de sistemas de uno o más componentes. El objetivo de éstas es además de mantener la confiabilidad del componente o sistema, ahorrar costos totales de mantenimiento. En el Capítulo 2 se vió que las actividades del mantenimiento preventivo prolongan la vida de los componentes, reduciendo el número de fallas e incrementando el $M T B F$ del sistema. Realizar mantenimiento preventivo a los sistemas les permitirá a éstos mantenerse en buenas condiciones para conseguir un buen rendimiento y los estándares de calidad. 
Las políticas de mantenimiento preventivo que se expusieron a lo largo de este trabajo indican que la confiabilidad es un factor clave para determinar el costo del ciclo de vida de un sistema. Mejorar la confiabilidad de un sistema cuesta dinero, pero se justifica económicamente si la disminución del costo de falla es, al menos, igual al aumento del costo debido a la mejora de la confiabilidad.

Tanto para sistemas de un solo componente como para aquellos de múltiples componentes, la política de reemplazo basado en la edad es la que genera el intervalo de reemplazo más largo, a diferencia de la política de reemplazo a intervalos constantes, reduciendo con ello el número de paradas del sistema, las cuales afectan la disponibilidad del mismo. La única desventaja de esta política es que se tiene que llevar un registro de los tiempos de las falla para calcular la edad del sistema, cosa que no sucede en la política de reemplazo a intervalos constantes, pues en esta otra los reemplazos preventivos se realizan en intervalos de tiempos fijos, sin tener en cuenta la edad del sistema.

Con respecto a los modelos de distribución de probabilidad que más se utilizan en el área de confiabilidad, podemos concluir que la distribución Weibull es la que más se utiliza en la práctica; ya que además de ser muy fácil de manejar, nos permite representar la tasa de falla en las tres zonas de la Curva de la Bañera, dependiendo del valor del parámetro de forma. Recordemos que para el caso de la distribución Exponencial, la cual se caracteriza por tener una tasa de falla constante (típica de los sistemas electrónicos) las políticas de mantenimiento preventivo demostraron no ser una buena estrategia, pues la aplicación de éstas solo genera pérdida de tiempo y dinero.

En lo que se refiere al ejemplo del elevador que se presentó en el Capítulo 3, se consideró que tanto la distribución Normal como la Weibull, se ajustan bastante bien a los datos, aunque el costo computacional es menor cuando se usa la distribución Weibull. Hay que recalcar que éstas dos distribuciones se ajustaron a un número reducido de datos. De hecho es un tanto difícil obtener los datos de falla de un sistema real, pues muchas veces los encargados de éstos llevan un registro de las fallas de los diferentes sistemas que mantienen y anotan sólo los datos. A pesar de esta limitación, se pudieron aplicar las políticas de mantenimiento preventivo y se vió que el tipo de mantenimiento que resulta óptimo es el de edad a 14 días con un costo de $\$ 50.48$ que es el mínimo. El tiempo medio entre fallas es de 32.68 días, el cual es mejor que el que se tenía con mantenimiento programado cada 30 días, el cual genera un costo de $\$ 58.31$.

El estudio de las políticas de mantenimiento para sistemas en serie, paralelo y mixto permitió ver que el intervalo de reemplazo óptimo es menor cuando se trata de un sistema en serie; el intervalo empieza a crecer cuando los componentes forman parte de una configuración mixta, y es mayor si los componentes forman parte de una configuración en paralelo.

Independientemente de si el sistema está formado por uno o más componentes y de la configuración que se considere, la política de mantenimiento preventivo basado en la edad siempre es mejor que la política de mantenimiento a intervalos constantes. 
Una buena alternativa de mantenimiento preventivo a la configuración en serie es la política de mantenimiento oportunista. Aquí la falla de un componente ocasiona la falla del sistema; en este momento se reemplaza el componente que falló y se evalúa la decisión de reemplazar los componentes que no fallaron, esto en base a la degradación de los componentes y la comparación de los costos de reemplazo por falla y reemplazo programado. Para ilustrar esta política empleamos la Simulación Monte Carlo para simular el comportamiento real de un sistema en serie formado por dos componentes, cuyo tiempo de falla es modelado con la distribución Weibull. Se calcularon los costos totales de mantenimiento para diferentes planes de mantenimiento preventivo y se seleccionó el plan que generó los menores costos. Los costos que se obtuvieron en el plan de mantenimiento óptimo fueron menores a los que se obtuvieron cuando se realiza el reemplazo conjunto de los dos componentes.

Queda pendiente el estudio de las políticas de mantenimiento preventivo para sistemas con más de dos componentes con diferente tasa de falla, suponiendo que tras la falla del sistema, sólo es reemplazado el componente que falló, en vez de reemplazar el sistema completo. Este otro procedimiento permitirá generar un ahorro en los costos totales de reemplazo preventivo.

Para finalizar, cabe mencionar que estas políticas serán de utilidad, para aquellas empresas que cuenten con sistemas, para los cuales, una falla inesperada del sistema le representa una gran pérdida de producción o se tengan consecuencias catastróficas; como por ejemplo: las aeronáuticas, las petroquímicas y generadoras de energía, por mencionar algunas. 


\section{Bibliografía}

[1] Duncan, A. J. (1996).Control de Calidad y Estadística Industrial. Richard D. Irwing, Inc., México.

[2] Cristaldi, L., Lazzaroni, M. and Catelani, M. (2011). Reliability Engineering. Springer, Italy.

[3] Tobias, P. A. and Trindade, D. C. (1995). Applied Reliability. Thomson Publishing Inc.

[4] Crowder, M. J., Klimber, A. C., Smith, R. L. and Sweeting, T. J. (1991). Statistical Analysis of Reliability Data. Chapman \& Hall, London .

[5] Crespo, A. M. (2007). The Maintenance Management Framework: models and methods for complex systems maintenance. Springer Series in Reliability Engineering.

[6] Redondo, J. C. (2007). Un Modelo Matemático Óptimo de Mantenimiento y Fiabilidad Aplicado a la Aviación Comercial. Tesis Doctoral, Universidad Nacional de Educación a Distancia, España.

[7] Barlow, R. E. and Frank P. (1996). Mathematical Theory of Reliability. Society for Industrial and Applied Mathematics.

[8] Modarres, M. K., Mark and Krivtsov, V. (1999). Reliability Engineering and Risk Analysis. Marcel Dekker, Inc., New York .

[9] Dodson, B. and Nolan, D. (2002). Reliability Engineering Handbook. QA Publishing, LLC.

[10] Laggoune, R., Chateauneuf, A. and Aissani, D. (2009). Opportunistic policy for optimal preventive maintenance of a multi-component system in continuous operating units. Computers \& Chemical Engineering, 33, 1499-1510.

[11] Campbell, J. D. and Jardine, A. K. S. (2001). Maintenance Excellence: Optimizing Equipment Life-Cycle Decisions. New York: Marcel Dekker.

[12] Smith, P. J. Analysis of Failure and Survival Data. (2002). Chapman \& Hall. 
[13] Nakawa, T. (2005). Maintenance Theory of Reliability. Springer Series in Reliability Engineering, London.

[14] Wang, H. and Pham, H. (2006). Reliability and Optimal Maintenance. Springer Series in Reliability Engineering, London. 